\title{
O-Benzoylhydroxylamines as Alkyl Nitrene Precursors: Synthesis of Saturated N-Heterocycles from Primary Amines
}

\author{
Hidetoshi Noda, ${ }^{*}$ Yasuko Asada and Masakatsu Shibasaki ${ }^{\star}$ \\ Institute of Microbial Chemistry (BIKAKEN), Tokyo, Japan \\ Email: hnoda@bikaken.or.jp; mshibasa@bikaken.or.jp
}

Table of Contents

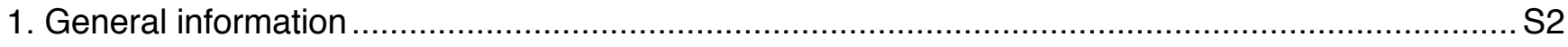

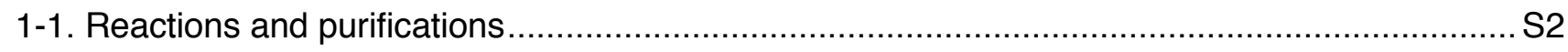

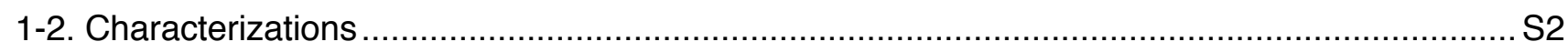

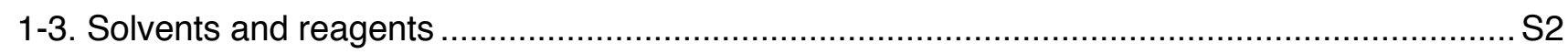

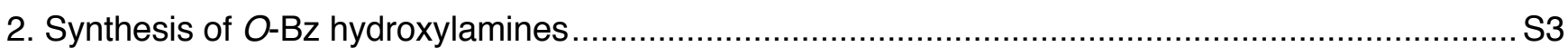

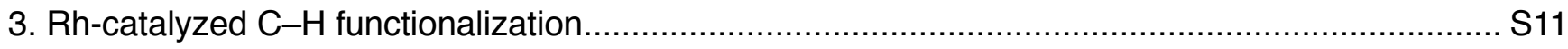

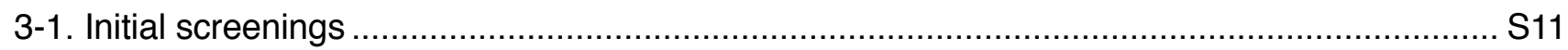

3-2. Substrate scope for pyrrolidine synthesis ................................................................ 112

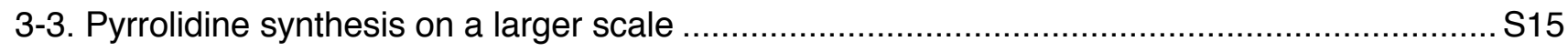

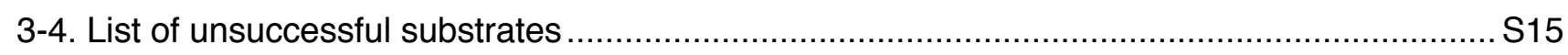

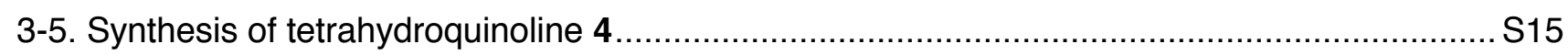

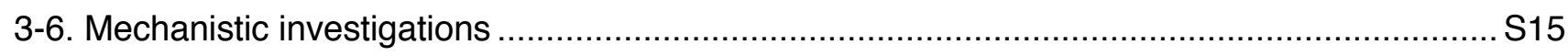

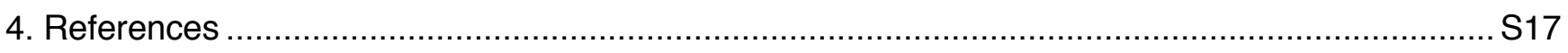

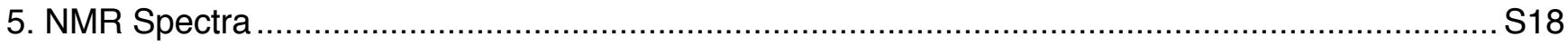




\section{General information}

\section{1-1. Reactions and purifications}

Unless otherwise noted, all reactions were carried out under an air atmosphere and were stirred with Teflon-coated magnetically stirred bars. All work-up and purification procedures were carried out with reagent-grade solvents under ambient atmosphere. Thin layer chromatography (TLC) was performed on Merck TLC plates $(0.25 \mathrm{~mm})$ precoated with silica gel 60 F254 and visualized by UV quenching and staining with ninhydrin or KMnO4. Normal phase flash column chromatography was performed on a Biotage Isolera Spektra One.

\section{1-2. Characterizations}

Infrared (IR) spectra were recorded on a HORIBA FT210 Fourier transform infrared spectrophotometer. NMR spectra were recorded on a Bruker AVANCE III HD400 NMR spectrometers. Chemical shifts $(\delta)$ are given in ppm relative to residual solvent peaks. ${ }^{1}$ Data for ${ }^{1} \mathrm{H}$ NMR are reported as follows: chemical shift (multiplicity, coupling constants where applicable, number of hydrogens). Abbreviations are as follows: s (singlet), $d$ (doublet), $t$ (triplet), $\mathrm{dd}$ (doublet of doublet), dt (doublet of triplet), ddd (doublet of doublet of doublet), q (quartet), m (multiplet), br (broad). High-resolution mass spectra (ESI TOF (+)) were measured on a Thermo Fisher Scientific LTQ Orbitrap XL.

\section{1-3. Solvents and reagents}

Unless otherwise noted, materials were purchased from commercial suppliers (TCI, Merck-Sigma-Aldrich, CombiBlocks, Enamine, BLD Pharmatech) and were used without further purification. Anhydrous $\mathrm{MeOH}$ were purchased from commercial suppliers. THF, $\mathrm{CH}_{2} \mathrm{Cl}_{2}$, and $\mathrm{CH}_{3} \mathrm{CN}$ were purified by passing through a solvent purification system (Glass Contour). HFIP and Rh2(esp)2 were purchased from Sigma-Aldrich, and used as received. 


\section{Synthesis of $O-B z$ hydroxylamines}

\begin{tabular}{|ccc|}
\hline $\mathrm{R}-\mathrm{NH}_{2}$ & $\mathrm{BzO}-\mathrm{OBz}$ & $\mathrm{Cs}_{2} \mathrm{CO}_{3}$ (3 equiv) \\
& $\begin{array}{c}\mathrm{CH}_{2} \mathrm{Cl}_{2} \\
2 \text { equiv }\end{array}$ & $\mathrm{R}-\mathrm{NH}$ \\
& $23^{\circ} \mathrm{C}$ & $\mathrm{OBz}$ \\
\hline
\end{tabular}

General procedure A: By following the reported procedure, ${ }^{2}$ to benzoyl peroxide (2.0 equiv) and $\mathrm{Cs}_{2} \mathrm{CO}_{3}$ (3.0 equiv) was added $\mathrm{CH}_{2} \mathrm{Cl}_{2}(0.1 \mathrm{M})$. The heterogeneous mixture was stirred for $2 \mathrm{~h}$ at $23^{\circ} \mathrm{C}$, and amine (1.0 equiv) was added. The mixture was further stirred for $14 \mathrm{~h}$. After the addition of water, the aqueous phase was extracted with $\mathrm{CH}_{2} \mathrm{Cl}_{2}$. The combined organic layers were washed with brine, dried over $\mathrm{Na}_{2} \mathrm{SO}_{4}$ and removed under reduced pressure. The crude product was purified by silica gel column chromatography, eluting with hexane/EtOAc to afford O-Bz hydroxylamines.

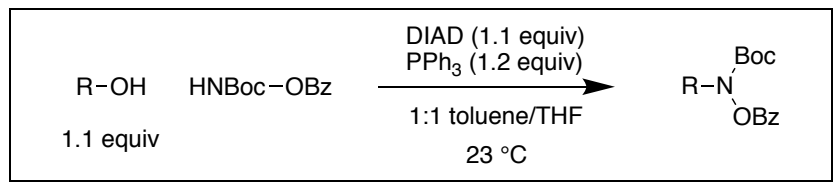

General procedure B: To a solution of alcohol (1.1 equiv), BocNHOBz, and $\mathrm{Ph}_{3} \mathrm{P}$ (1.2 equiv) in 1:1 toluene/THF (0.3 M) was added DIAD (1.1 equiv) slowly at $0{ }^{\circ} \mathrm{C}$ (ice bath). The solution was warmed to $23{ }^{\circ} \mathrm{C}$ and stirred until the hydroxylamine was fully consumed (typically 2-3 hours). The volatile was removed under reduced pressure to give a crude material, which was purified by silica gel column chromatography, eluting with hexane/EtOAc to afford protected hydroxylamine.

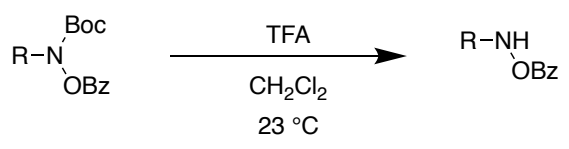

General procedure C: To a solution of Boc-protected hydroxylamine in $\mathrm{CH}_{2} \mathrm{Cl}_{2}(0.5 \mathrm{M})$ was added TFA (1:1 volume to $\mathrm{CH}_{2} \mathrm{Cl}_{2}$ ) at $0{ }^{\circ} \mathrm{C}$ (ice bath). The resulting solution was warmed to $23{ }^{\circ} \mathrm{C}$ and stirred until the substrate was fully consumed (typically $<1$ hour). After the dilution with $\mathrm{CH}_{2} \mathrm{Cl}_{2}$, the mixture was basified with sat aq $\mathrm{NaHCO}_{3}$. The aqueous phase was extracted with $\mathrm{CH}_{2} \mathrm{Cl}_{2}$. The combined organic layers were washed with brine, dried over $\mathrm{Na}_{2} \mathrm{SO}_{4}$, and removed under reduced pressure. The crude residue was purified by silica gel column chromatography, eluting with hexane/EtOAc to afford the corresponding O-Bz hydroxylamines.

O-Benzoyl-N-(4-phenylbutyl)hydroxylamine (1a): Prepared by the general procedure A from 4-phenylbutan-1-

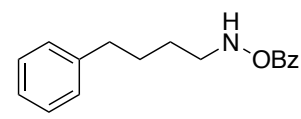

amine ( $2.00 \mathrm{~mL}, 12 \mathrm{mmol})$, purified by silica gel column chromatography (hexane/EtOAc), and isolated as a colorless oil ( $2.39 \mathrm{~g}, 74 \%$ yield). IR (thin film): $v 3238,3062,3025,2937,2858,1718$, 1451, 1269, 1085, 1065, $1024 \mathrm{~cm}^{-1}{ }^{1}{ }^{1} \mathrm{H}$ NMR (400 MHz, CDCl $): \delta 8.03-8.00(\mathrm{~m}, 2 \mathrm{H}), 7.62-7.57(\mathrm{~m}$, 1H), 7.48-7.44 (m, 2H), 7.30-7.26 (m, 2H), 7.20-7.17 (m, 3H), $6.43(\mathrm{brs}, 1 \mathrm{H}), 3.18(\mathrm{t}, J=6.9 \mathrm{~Hz}, 2 \mathrm{H}), 2.67(\mathrm{t}, J=7.4 \mathrm{~Hz}$, 2H), 1.79-1.67 (m, 4H); ${ }^{13} \mathrm{C}$ NMR (100 MHz, $\left.\mathrm{CDCl}_{3}\right): \delta$ 166.9, 142.2, 133.5, 129.5, 128.7, 128.5, 128.5, 128.4, 126.0, 52.4, 35.8, 28.9, 26.8; HRMS (ESI) $m / z:[\mathrm{M}+\mathrm{H}]^{+}$Calcd for $\mathrm{C}_{17} \mathrm{H}_{20} \mathrm{O}_{2} \mathrm{~N}$ 270.1489; Found 270.1494. 
tert-Butyl (benzoyloxy)(4-(p-tolyl)butyl)carbamate (Boc-1b): Prepared by the general procedure B from 4-(pBoc tolyl)butan-1-ol $(1.23 \mathrm{~g}, 7.49 \mathrm{mmol})$, purified by silica gel column chromatography (hexane/EtOAc), and isolated as a colorless oil (2.64 g, 92\% yield). IR (thin film): $v 2977,2934$, 2860, 1765, 1714, 1452, 1392, 1368, 1241, 1157, $1013 \mathrm{~cm}^{-1}$; 1 H NMR (400 MHz, $\left.\mathrm{CDCl}_{3}\right): \delta 8.08$ $8.02(\mathrm{~m}, 2 \mathrm{H}), 7.66-7.58(\mathrm{~m}, 1 \mathrm{H}), 7.50-7.44(\mathrm{~m}, 2 \mathrm{H}), 7.08-7.04(\mathrm{~m}, 4 \mathrm{H}), 3.79-3.65(\mathrm{~m}, 2 \mathrm{H}), 2.69-2.54(\mathrm{~m}, 2 \mathrm{H}), 2.30(\mathrm{~s}, 3 \mathrm{H})$, 1.73-1.66 (m, 4H), 1.45 (s, 9H); ${ }^{13} \mathrm{C}$ NMR (100 MHz, $\left.\mathrm{CDCl}_{3}\right): \delta 164.8,155.1,139.1,135.3,133.9,130.0,129.1,128.7,128.4$, 127.9, 82.4, 50.6, 35.1, 28.6, 28.3, 26.9, 21.1; HRMS (ESI) $\mathrm{m} / \mathrm{z}$ : $[\mathrm{M}+\mathrm{Na}]^{+}$Calcd for $\mathrm{C}_{23} \mathrm{H}_{29} \mathrm{O}_{4} \mathrm{NNa}$ 406.1989; Found 406.1995.

$O$-Benzoyl- $N$-(4-( $p$-tolyl)butyl)hydroxylamine (1b): Prepared by the general procedure C from tert-butyl

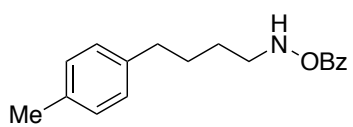
(benzoyloxy)(4-(p-tolyl)butyl)carbamate $(1.92 \mathrm{~g}, 5.01 \mathrm{mmol})$, purified by silica gel column chromatography (hexane/EtOAc), and isolated as a colorless oil (894 mg, 63\% yield). IR (thin film): $v$ 3236, 2937, 2858, 1719, 1451, 1270, 1092, 1065, $1024 \mathrm{~cm}^{-1}$; ${ }^{1} \mathbf{H}$ NMR (400 MHz, $\left.\mathrm{CDCl}_{3}\right): \delta$ 8.07-7.98 (m, 2H), 7.64-7.56 (m, 1H), 7.51-7.42 (m, 2H), 7.12-7.07 (m, 5H), 3.17 (t, J=6.9 Hz, 2H), 2.64 (t, J= $7.3 \mathrm{~Hz}, 2 \mathrm{H}), 2.33$ (s, 3H), 1.81-1.61 (m, 4H); ${ }^{13} \mathrm{C}$ NMR (100 MHz, $\left.\mathrm{CDCl}_{3}\right): \delta$ 167.0, 139.0, 135.3, 133.4, 129.5, 129.1, 128.6, 128.5, 128.4, 52.5, 35.3, 29.0, 26.8, 21.1; HRMS (ESI) $m / z:[\mathrm{M}+\mathrm{Na}]^{+}$Calcd for $\mathrm{C}_{18} \mathrm{H}_{21} \mathrm{O}_{2} \mathrm{NNa}$ 306.1465; Found 306.1465.

tert-Butyl (benzoyloxy)(4-(4-methoxyphenyl)butyl)carbamate (Boc-1c): Prepared by the general procedure B from

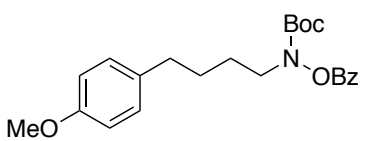
4-(4-methoxyphenyl)butan-1-ol (717 mg, $3.98 \mathrm{mmol})$, purified by silica gel column chromatography (hexane/EtOAc), and isolated as a colorless oil (1.27 g, 80\% yield). IR (thin film): $v$ 2977, 2935, 1765, 1714, 1512, 1368, 1245, 1157, 1039, $1013 \mathrm{~cm}^{-1}$; ${ }^{1} \mathbf{H}$ NMR (400 $\left.\mathrm{MHz}, \mathrm{CDCl}_{3}\right): \delta 8.07-8.01(\mathrm{~m}, 2 \mathrm{H}), 7.66-7.58(\mathrm{~m}, 1 \mathrm{H}), 7.50-7.44(\mathrm{~m}, 2 \mathrm{H}), 7.11-7.05(\mathrm{~m}, 2 \mathrm{H}), 6.83-6.77(\mathrm{~m}, 2 \mathrm{H}), 3.77(\mathrm{~s}$, $3 \mathrm{H}), 3.72(\mathrm{t}, J=6.5 \mathrm{~Hz}, 2 \mathrm{H}), 2.62-2.55(\mathrm{~m}, 2 \mathrm{H}), 1.74-1.65(\mathrm{~m}, 4 \mathrm{H}), 1.45(\mathrm{~s}, 9 \mathrm{H}) ;{ }^{13} \mathrm{C}$ NMR $\left(100 \mathrm{MHz}, \mathrm{CDCl}_{3}\right): \delta 164.8$, $157.9,155.1,134.3,133.9,130.0,129.4,128.7,127.9,113.9,82.4,55.4,50.6,34.6,28.7,28.3,26.8$; HRMS (ESI) $m / z:$ [M + $\mathrm{Na}]^{+}$Calcd for $\mathrm{C}_{23} \mathrm{H}_{29} \mathrm{O}_{5} \mathrm{NNa}$ 422.1938; Found 422.1946.

O-Benzoyl-N-(4-(4-methoxyphenyl)butyl)hydroxylamine (1c): Prepared by the general procedure C from tert-butyl<smiles>COc1ccc(CCCCN[R6](=O)c2ccccc2)cc1</smiles>
(benzoyloxy)(4-(4-methoxyphenyl)butyl)carbamate $(964 \mathrm{mg}, 2.41 \mathrm{mmol})$, purified by silica gel column chromatography (hexane/EtOAc), and isolated as a colorless oil (512 mg, 71\% yield). IR (thin film): $v$ 3235, 2936, 2857, 1718, 1511, 1451, 1270, 1245, 1177, 1092, 1065, 1025 $\mathrm{cm}^{-1} ;{ }^{1} \mathrm{H}$ NMR $\left(400 \mathrm{MHz}, \mathrm{CDCl}_{3}\right): \delta 8.04-7.97(\mathrm{~m}, 2 \mathrm{H}), 7.65-7.55(\mathrm{~m}, 1 \mathrm{H}), 7.46(\mathrm{dd}, J=7.1,8.4 \mathrm{~Hz}, 2 \mathrm{H}), 7.13-7.05(\mathrm{~m}$, 2H), 6.86-6.78 (m, 2H), $3.78(\mathrm{~s}, 3 \mathrm{H}), 3.17(\mathrm{t}, J=6.7 \mathrm{~Hz}, 2 \mathrm{H}), 2.61(\mathrm{t}, J=7.2 \mathrm{~Hz}, 2 \mathrm{H}), 1.80-1.62(\mathrm{~m}, 4 \mathrm{H}) ;{ }^{13} \mathrm{C}$ NMR (100 $\left.\mathrm{MHz}, \mathrm{CDCl}_{3}\right): \delta$ 167.0, 157.9, 134.2, 133.5, 129.5, 129.4, 128.7, 128.5, 113.9, 55.4, 52.5, 34.8, 29.1, 26.7; HRMS (ESI) $m / z$ : $[\mathrm{M}+\mathrm{H}]^{+}$Calcd for $\mathrm{C}_{18} \mathrm{H}_{22} \mathrm{O}_{3} \mathrm{~N}$ 300.1594; Found 300.1599 . 
tert-Butyl (benzoyloxy)(4-(4-cyanophenyl)butyl)carbamate (Boc-1d): Prepared by the general procedure B from 4(n) (4-hydroxybutyl)benzonitrile (315 $\mathrm{mg}, 1.80 \mathrm{mmol}$ ), purified by silica gel column chromatography (hexane/EtOAc), and isolated as a colorless oil (653 mg, 92\% yield). IR (thin film): $v$ 2978, 2936, 2864, 2227, 1764, 1714, 1452, 1393, 1368, 1242, 1157, $1013 \mathrm{~cm}^{-1} ;{ }^{1} \mathbf{H}$ NMR (400 MHz, $\left.\mathrm{CDCl}_{3}\right): \delta$ 8.05-7.99 (m, 2H), 7.67-7.59 (m, 1H), 7.56-7.51 (m, 2H), 7.50-7.44 (m, 2H), 7.30-7.23 (m, $2 \mathrm{H}), 3.72(\mathrm{t}, J=6.8 \mathrm{~Hz}, 2 \mathrm{H}), 2.70(\mathrm{t}, J=7.5 \mathrm{~Hz}, 2 \mathrm{H}), 1.81-1.59(\mathrm{~m}, 4 \mathrm{H}) ;{ }^{13} \mathrm{C} \mathbf{N M R}\left(100 \mathrm{MHz}, \mathrm{CDCl}_{3}\right): \delta 164.8,155.0,147.9$, 134.0, 132.3, 129.9, 129.3, 128.8, 127.7, 119.2, 109.8, 82.5, 50.2, 35.6, 28.3, 27.9, 26.8; HRMS (ESI) $m / z:[\mathrm{M}+\mathrm{Na}]^{+}$Calcd for $\mathrm{C}_{23} \mathrm{H}_{26} \mathrm{O}_{4} \mathrm{~N}_{2} \mathrm{Na}$ : 417.1785; Found 417.1788.

4-(4-((Benzoyloxy)amino)butyl)benzonitrile (1d): Prepared by the general procedure $C$ from tert-butyl 舟C (benzoyloxy)(4-(4-cyanophenyl)butyl)carbamate $(664 \mathrm{mg}, 1.68 \mathrm{mmol})$, purified by silica gel column chromatography (hexane/EtOAc), and isolated as a colorless oil (469 mg, 95\% yield). IR (thin film): $v$ 3239, 2940, 2861, 2226, 1719, 1606, 1451, 1271, 1092, 1065, $1024 \mathrm{~cm}^{-1} ;{ }^{1} \mathbf{H}$ NMR

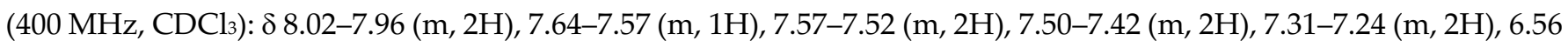
$(\mathrm{s}, 1 \mathrm{H}), 3.17(\mathrm{t}, J=6.9 \mathrm{~Hz}, 2 \mathrm{H}), 2.72(\mathrm{t}, J=7.5 \mathrm{~Hz}, 2 \mathrm{H}), 1.85-1.72(\mathrm{~m}, 2 \mathrm{H}), 1.73-1.58(\mathrm{~m}, 2 \mathrm{H}) ;{ }^{13} \mathrm{C}$ NMR $\left(100 \mathrm{MHz}, \mathrm{CDCl}_{3}\right)$ : $\delta$ 166.9, 147.8, 133.6, 132.3, 129.5, 129.3, 128.7, 128.3, 119.2, 109.9, 52.2, 35.9, 28.3, 26.7; HRMS (ESI) $m / z:[M+H]^{+}$Calcd for $\mathrm{C}_{18} \mathrm{H}_{19} \mathrm{O}_{2} \mathrm{~N}_{2} 295.1441$; Found 295.1447.

Methyl 4-(4-((benzoyloxy)(tert-butoxycarbonyl)amino)butyl)benzoate (Boc-1e): Prepared by the general

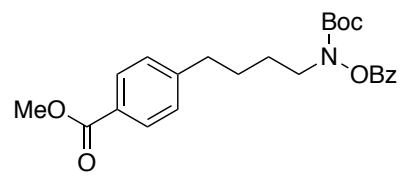
procedure B from methyl 4-(4-hydroxybutyl)benzoate $(1.22 \mathrm{~g}, 5.88 \mathrm{mmol})$, purified by silica gel column chromatography (hexane/EtOAc), and isolated as a colorless oil ( $2.23 \mathrm{~g}$, 89\% yield). IR (thin film): $v 2978,2943,2863,1765,1721,1368,1280,1242,1157,1108,1013$ $\mathrm{cm}^{-1} ;{ }^{1} \mathrm{H}$ NMR $\left(400 \mathrm{MHz}, \mathrm{CDCl}_{3}\right): \delta 8.06-8.00(\mathrm{~m}, 2 \mathrm{H}), 7.96-7.89(\mathrm{~m}, 2 \mathrm{H}), 7.64-7.58(\mathrm{~m}$, 1H), 7.50-7.43 (m, 2H), 7.25-7.20 (m, 2H), $3.89(\mathrm{~s}, 3 \mathrm{H}), 3.72(\mathrm{t}, J=6.8 \mathrm{~Hz}, 2 \mathrm{H}), 2.70(\mathrm{t}, J=7.4 \mathrm{~Hz}, 2 \mathrm{H}), 1.83-1.62(\mathrm{~m}, 4 \mathrm{H})$, 1.45 (s, 9H); ${ }^{13} \mathrm{C}$ NMR (100 MHz, $\left.\mathrm{CDCl}_{3}\right): \delta$ 167.2, 164.8, 155.0, 147.8, 133.9, 130.0, 129.8, 128.7, 128.6, 128.0, 127.8, 82.5, 52.1, 50.4, 35.5, 28.3, 28.1, 26.8; HRMS (ESI) $m / z$ : [M + Na $]^{+}$Calcd for $\mathrm{C}_{24} \mathrm{H}_{29} \mathrm{O}_{6} \mathrm{NNa}$ 450.1887; Found 450.1893.

Methyl 4-(4-((benzoyloxy)amino)butyl)benzoate (1e): Prepared by the general procedure C from methyl 4-(4-<smiles>COC(=O)NCCCCc1ccc(C(=O)OC)cc1</smiles((benzoyloxy)(tert-butoxycarbonyl)amino)butyl)benzoate (1.43 g, $3.33 \mathrm{mmol})$, purified by silica gel column chromatography (hexane/EtOAc), and isolated as a colorless oil (1.04 g, 96\% yield). IR (thin film): $v$ 3237, 2945, 2860, 1719, 1609, 1451, 1434, 1277, 1178, 1108, 1065, $1022 \mathrm{~cm}^{-1}$; ${ }^{1} \mathrm{H}$ NMR (400 MHz, CDCl $): \delta 8.03-7.97(\mathrm{~m}, 2 \mathrm{H}), 7.96-7.91(\mathrm{~m}, 2 \mathrm{H}), 7.62-7.54(\mathrm{~m}, 1 \mathrm{H}), 7.49-7.41(\mathrm{~m}$, 2H), 7.29-7.20 (m, 2H), $3.90(\mathrm{~s}, 3 \mathrm{H}), 3.17(\mathrm{t}, J=6.9 \mathrm{~Hz}, 2 \mathrm{H}), 2.71(\mathrm{t}, J=7.5 \mathrm{~Hz}, 2 \mathrm{H}), 1.85-1.73(\mathrm{~m}, 2 \mathrm{H}), 1.72-1.61(\mathrm{~m}, 2 \mathrm{H})$; ${ }^{13} \mathrm{C}$ NMR (100 MHz, $\left.\mathrm{CDCl}_{3}\right): \delta 167.2,166.9,147.7,133.5,129.8,129.5,128.7,128.6,128.4,128.0,52.3,52.1,35.8,28.5$, 26.8; HRMS (ESI) $m / z:[\mathrm{M}+\mathrm{H}]^{+}$Calcd for $\mathrm{C}_{19} \mathrm{H}_{22} \mathrm{O}_{4} \mathrm{~N}$ 328.1543; Found 328.1549. 
tert-Butyl (benzoyloxy)(4-(3-methoxyphenyl)butyl)carbamate (Boc-1f): Prepared by the general procedure B from

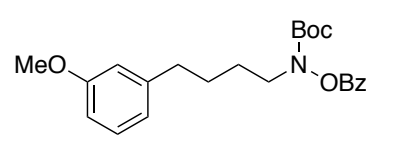

4-(3-methoxyphenyl)butan-1-ol (1.23 g, $6.81 \mathrm{mmol})$, purified by silica gel column chromatography (hexane/EtOAc), and isolated as a colorless oil ( $1.82 \mathrm{~g}, 67 \%$ yield). IR (thin film): $v$ 2977, 2937, 1764, 1714, 1600, 1453, 1368, 1258, 1154, $1041 \mathrm{~cm}^{-1}$; ${ }^{1} \mathbf{H}$ NMR (400 MHz, $\left.\mathrm{CDCl}_{3}\right): \delta 8.09-8.02(\mathrm{~m}, 2 \mathrm{H}), 7.66-7.57(\mathrm{~m}, 1 \mathrm{H}), 7.51-7.42(\mathrm{~m}, 2 \mathrm{H}), 7.21-7.12(\mathrm{~m}, 1 \mathrm{H}), 6.80-6.69(\mathrm{~m}, 3 \mathrm{H}), 3.78(\mathrm{~s}, 3 \mathrm{H})$, $3.72(\mathrm{t}, J=6.5 \mathrm{~Hz}, 2 \mathrm{H}), 2.63(\mathrm{t}, J=7.1 \mathrm{~Hz}, 2 \mathrm{H}), 1.79-1.65(\mathrm{~m}, 4 \mathrm{H}), 1.46(\mathrm{~s}, 9 \mathrm{H}) ;{ }^{13} \mathrm{C}$ NMR $\left(100 \mathrm{MHz}, \mathrm{CDCl}_{3}\right): \delta 164.8$, 159.8, 155.0, 143.9, 133.9, 130.0, 129.4, 128.7, 127.8, 121.0, 114.2, 111.2, 82.4, 55.2, 50.5, 35.6, 28.3, 28.3, 26.9; HRMS (ESI) $m / z:[\mathrm{M}+\mathrm{Na}]^{+}$Calcd for $\mathrm{C}_{23} \mathrm{H}_{29} \mathrm{O}_{5} \mathrm{NNa}$ 422.1938; Found 422.1943.

O-Benzoyl-N-(4-(3-methoxyphenyl)butyl)hydroxylamine (1f): Prepared by the general procedure C from tert-butyl<smiles>COc1cccc(CCCCNC(=O)Oc2ccccc2)c1</smiles>
(benzoyloxy)(4-(3-methoxyphenyl)butyl)carbamate (1.5 g, $3.8 \mathrm{mmol})$, purified by silica gel column chromatography (hexane/EtOAc), and isolated as a colorless oil (898 mg, 79\% yield). IR (thin film): $v$ 3236, 2938, 2859, 1719, 1600, 1584, 1487, 1452, 1268, 1152, 1091, 1065 , 1044, $1025 \mathrm{~cm}^{-1}$; ${ }^{1} \mathrm{H}$ NMR (400 MHz, $\left.\mathrm{CDCl}_{3}\right): \delta 8.05-7.98$ (m, 2H), $7.59(\mathrm{ddt}, J=7.9,6.9,1.3 \mathrm{~Hz}, 1 \mathrm{H}), 7.50-7.42(\mathrm{~m}, 2 \mathrm{H})$, 7.23-7.15 (m, 1H), 6.81-6.76 (m, 1H), 6.75-6.71 (m, 2H), $3.79(\mathrm{~s}, 3 \mathrm{H}), 3.17(\mathrm{t}, J=6.9 \mathrm{~Hz}, 2 \mathrm{H}), 2.65(\mathrm{t}, J=7.4 \mathrm{~Hz}, 2 \mathrm{H})$, 1.84-1.63 (m, 4H); ${ }^{13} \mathrm{C}$ NMR (100 MHz, $\left.\mathrm{CDCl}_{3}\right): \delta 167.0,159.8,143.8,133.5,129.5,129.4,128.6,128.5,121.0,114.3,111.2$, 55.2, 52.5, 35.8, 28.8, 26.8; HRMS (ESI) $m / z:[\mathrm{M}+\mathrm{H}]^{+}$Calcd for $\mathrm{C}_{18} \mathrm{H}_{22} \mathrm{O}_{3} \mathrm{~N}$ 300.1594; Found 300.1601.

Methyl 2-(4-((benzoyloxy)(tert-butoxycarbonyl)amino)butyl)benzoate (Boc-1g): Prepared by the general $\mathrm{MeO} Y \mathrm{O} \quad \mathrm{Boc} \quad$ procedure $\mathrm{B}$ from methyl 2-(4-hydroxybutyl)benzoate $(1.02 \mathrm{~g}, 4.90 \mathrm{mmol})$, purified by silica gel column chromatography (hexane/EtOAc), and isolated as a colorless oil (1.99 g, 95\% yield). IR (thin film): $v$ 2977, 2947, 2865, 1764, 1721, 1600, 1451, 1368, 1257, 1158, 1081, 1012 $\mathrm{cm}^{-1} ;{ }^{1} \mathrm{H}$ NMR (400 MHz, $\left.\mathrm{CDCl}_{3}\right): \delta 8.10-8.02(\mathrm{~m}, 2 \mathrm{H}), 7.89-7.82(\mathrm{~m}, 1 \mathrm{H}), 7.66-7.57(\mathrm{~m}, 1 \mathrm{H}), 7.51-7.44(\mathrm{~m}, 2 \mathrm{H}), 7.42-$ $7.35(\mathrm{~m}, 1 \mathrm{H}), 7.26-7.18(\mathrm{~m}, 2 \mathrm{H}), 3.87(\mathrm{~s}, 3 \mathrm{H}), 3.73(\mathrm{t}, J=6.6 \mathrm{~Hz}, 2 \mathrm{H}), 2.99(\mathrm{t}, J=7.2 \mathrm{~Hz}, 2 \mathrm{H}), 1.82-1.66(\mathrm{~m}, 4 \mathrm{H}), 1.45(\mathrm{~s}$, 9H); ${ }^{13} \mathrm{C}$ NMR (100 MHz, $\left.\mathrm{CDCl}_{3}\right): \delta 168.1,164.8,155.0,144.2,133.9,132.0,131.1,130.8,130.0,129.5,128.7,127.9,126.0$, 82.4, 52.0, 50.6, 34.1, 28.8, 28.3, 27.3; HRMS (ESI) $m / z$ : $[\mathrm{M}+\mathrm{Na}]^{+}$Calcd for $\mathrm{C}_{24} \mathrm{H}_{29} \mathrm{O}_{6} \mathrm{NNa}$ 450.1887; Found 450.1896.

Methyl 2-(4-((benzoyloxy)amino)butyl)benzoate (1g): Prepared by the general procedure C from methyl 2-(4-<smiles>COC(=O)NCCCCc1ccccc1</smiles((benzoyloxy)(tert-butoxycarbonyl)amino)butyl)benzoate $(1.01 \mathrm{~g}, 2.36 \mathrm{mmol})$, purified by silica gel column chromatography (hexane/EtOAc), and isolated as a colorless oil (757 mg, 98\% yield). IR (thin film): $v 3238,3063,2946,2862,1719,1450,1267,1094,1066 \mathrm{~cm}^{-1} ;{ }^{1} \mathbf{H}$ NMR (400 MHz, $\left.\mathrm{CDCl}_{3}\right): \delta 8.05-7.98(\mathrm{~m}, 2 \mathrm{H}), 7.90-7.85(\mathrm{~m}, 1 \mathrm{H}), 7.63-7.55(\mathrm{~m}, 1 \mathrm{H}), 7.49-7.37(\mathrm{~m}, 3 \mathrm{H}), 7.29-7.20(\mathrm{~m}, 2 \mathrm{H}), 3.88$ (s, 3H), 3.27-3.15 (m, 2H), 3.07-2.94 (m, 2H), 1.82-1.67 (m, 4H); ${ }^{13} \mathrm{C}$ NMR (100 MHz, CDCl $): \delta 168.1,166.9,144.2$, 133.5, 132.1, 131.1, 130.9, 129.5, 129.5, 128.7, 128.5, 126.1, 52.3, 52.1, 34.2, 29.1, 27.1; HRMS (ESI) $m / z:[M+H]^{+}$Calcd for $\mathrm{C}_{19} \mathrm{H}_{22} \mathrm{O}_{4} \mathrm{~N} 328.1543$; Found 328.1552. 
O-Benzoyl-N-(4-(pyridin-2-yl)butyl)hydroxylamine (1h): Prepared by the general procedure A from 4-(pyridin-2-

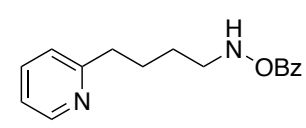

yl)butan-1-amine (465 mg, $3.1 \mathrm{mmol}$ ), purified by silica gel column chromatography (hexane/EtOAc), and isolated as a colorless oil (628 mg, 75\% yield). IR (thin film): $v$ 3235, 3063, 3008, 2941, 2861, 1719, 1590, 1474, 1451, 1434, 1271, 1091, 1066, $1024 \mathrm{~cm}^{-1}$; ${ }^{1} \mathbf{H}$ NMR (400 MHz,

$\left.\mathrm{CDCl}_{3}\right): \delta 8.52(\mathrm{ddd}, J=5.0,1.9,1.1 \mathrm{~Hz}, 1 \mathrm{H}), 8.04-7.97(\mathrm{~m}, 2 \mathrm{H}), 7.88(\mathrm{~s}, 1 \mathrm{H}), 7.64-7.54(\mathrm{~m}, 2 \mathrm{H}), 7.49-7.40(\mathrm{~m}, 2 \mathrm{H}), 7.16$ $(\mathrm{ddd}, J=7.8,7.5,1.1 \mathrm{~Hz}, 1 \mathrm{H}), 7.11(\mathrm{ddd}, J=7.5,5.0,1.1 \mathrm{~Hz}, 1 \mathrm{H}), 3.18(\mathrm{t}, J=7.1 \mathrm{~Hz}, 2 \mathrm{H}), 2.85(\mathrm{t}, J=7.6 \mathrm{~Hz}, 2 \mathrm{H}), 1.94-$ $1.82(\mathrm{~m}, 2 \mathrm{H}), 1.76-1.65$ (m, 2H); ${ }^{13} \mathrm{C}$ NMR (100 MHz, $\left.\mathrm{CDCl}_{3}\right): \delta$ 167.0, 161.7, 149.1, 136.8, 133.4, 129.5, 128.6, 128.6, 123.0, 121.3, 52.5, 37.9, 27.3, 27.0; HRMS (ESI) $m / z:[\mathrm{M}+\mathrm{H}]^{+}$Calcd for $\mathrm{C}_{16} \mathrm{H}_{19} \mathrm{O}_{2} \mathrm{~N}_{2}$ 271.1441; Found 271.1447.

O-Benzoyl-N-(5-methylhexan-2-yl)hydroxylamine (1i): Prepared by the general procedure A from 5-methylhexan2-amine $(276 \mathrm{mg}, 2.40 \mathrm{mmol})$, purified by silica gel column chromatography (hexane/EtOAc), and isolated as a colorless oil (463 mg, 82\% yield). IR (thin film): $v 3235,2956,2933,2869,1719,1452$,

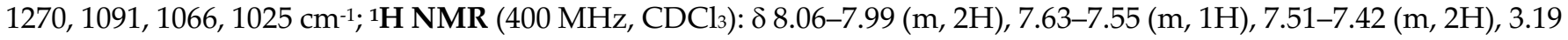
$(\mathrm{qt}, J=6.4,6.4 \mathrm{~Hz}, 1 \mathrm{H}), 1.74-1.50(\mathrm{~m}, 2 \mathrm{H}), 1.48-1.36(\mathrm{~m}, 1 \mathrm{H}), 1.34-1.24(\mathrm{~m}, 2 \mathrm{H}), 1.20(\mathrm{~d}, J=6.4 \mathrm{~Hz}, 3 \mathrm{H}), 0.90(\mathrm{~d}, J=6.7$ $\mathrm{Hz}, 6 \mathrm{H}) ;{ }^{13} \mathrm{C}$ NMR (100 MHz, $\left.\mathrm{CDCl}_{3}\right): \delta 167.0,133.4,129.5,128.7,128.6,57.3,35.1,31.9,28.3,22.8,22.6,18.3$; HRMS (ESI) $m / z:[\mathrm{M}+\mathrm{H}]^{+}$Calcd for $\mathrm{C}_{14} \mathrm{H}_{22} \mathrm{O}_{2} \mathrm{~N}$ 236.1645; Found 236.1649 .

O-Benzoyl-N-heptylhydroxylamine (1j): Prepared by the general procedure A from heptan-1-amine $(1.0 \mathrm{~mL}, 6.7$ mmol), purified by silica gel column chromatography (hexane/EtOAc), and isolated as a colorless oil (930 mg, 59\% yield). IR (thin film): $v$ 3237, 2928, 2857, 1720, 1452, 1270, 1091, 1066, $1024 \mathrm{~cm}^{-1} ;{ }^{1} \mathrm{H}$ NMR $\left(400 \mathrm{MHz}, \mathrm{CDCl}_{3}\right): \delta 8.06-7.99(\mathrm{~m}, 2 \mathrm{H}), 7.62-7.55(\mathrm{~m}, 1 \mathrm{H}), 7.49-7.42(\mathrm{~m}, 2 \mathrm{H}), 6.79(\mathrm{~s}, 1 \mathrm{H}), 3.21-$ $3.10(\mathrm{~m}, 2 \mathrm{H}), 1.70-1.56(\mathrm{~m}, 2 \mathrm{H}), 1.46-1.21(\mathrm{~m}, 8 \mathrm{H}), 0.95-0.81(\mathrm{~m}, 3 \mathrm{H}) ;{ }^{13} \mathrm{C}$ NMR $\left(100 \mathrm{MHz}, \mathrm{CDCl}_{3}\right): \delta 167.0,133.5,129.5$, 128.7, 128.6, 52.7, 31.9, 29.2, 27.3, 27.2, 22.7, 14.2; HRMS (ESI) $m / z$ : [M + Na $]^{+}$Calcd for $\mathrm{C}_{14} \mathrm{H}_{21} \mathrm{O}_{2} \mathrm{NNa} 258.1465$; Found 258.1467.

tert-Butyl (benzoyloxy)(5-phenylpentyl)carbamate (Boc-1k): Prepared by the general procedure B from 5Phenylpentan-1-ol $(504 \mathrm{mg}, 3.07 \mathrm{mmol})$, purified by silica gel column chromatography 2858, 1765, 1714, 1453, 1368, 1239, 1157, $1014 \mathrm{~cm}^{-1} ;{ }^{1} \mathrm{H}$ NMR (400 MHz, CDCl $): \delta 8.10-8.03(\mathrm{~m}, 2 \mathrm{H}), 7.66-7.58(\mathrm{~m}, 1 \mathrm{H})$, 7.52-7.44 (m, 2H), 7.30-7.23 (m, 2H), 7.19-7.13 (m, 3H), $3.68(\mathrm{t}, J=7.2 \mathrm{~Hz}, 2 \mathrm{H}), 2.67-2.55(\mathrm{~m}, 2 \mathrm{H}), 1.75-1.60(\mathrm{~m}, 4 \mathrm{H})$, 1.48-1.39 (m, 11H); ${ }^{13} \mathrm{C}$ NMR (100 MHz, $\left.\mathrm{CDCl}_{3}\right): \delta 164.8,155.0,142.6,133.9,130.0,128.7,128.5,128.4,127.9,125.8,82.4$, 50.7, 36.0, 31.2, 28.3, 27.3, 26.5; HRMS (ESI) $m / z$ : [M + Na] ${ }^{+}$Calcd for $\mathrm{C}_{23} \mathrm{H}_{29} \mathrm{O}_{4} \mathrm{NNa}$ 406.1989; Found 406.1990.

O-Benzoyl-N-(5-phenylpentyl)hydroxylamine (1k): Prepared by the general procedure C from tert-butyl (benzoyloxy)(5-phenylpentyl)carbamate (501 mg, $1.30 \mathrm{mmol})$, purified by silica gel column film): $v$ 3236, 3061, 3025, 2934, 2857, 1719, 1452, 1270, 1086, 1066, $1025 \mathrm{~cm}^{-1} ;{ }^{1} \mathbf{H}$ NMR (400 MHz, CDCl $): \delta 8.06-7.99$ Page $\mathrm{S} 7$ of $\mathrm{S} 60$ 
(m, 2H), 7.63-7.55 (m, 1H), 7.50-7.42 (m, 2H), 7.32-7.24 (m, 2H), 7.22-7.15 (m, 3H), $6.98(\mathrm{~s}, 1 \mathrm{H}), 3.15(\mathrm{t}, J=6.9 \mathrm{~Hz}, 2 \mathrm{H})$, $2.64(\mathrm{t}, J=7.8 \mathrm{~Hz}, 2 \mathrm{H}), 1.72-1.64(\mathrm{~m}, 4 \mathrm{H}), 1.53-1.40(\mathrm{~m}, 2 \mathrm{H}) ;{ }^{13} \mathrm{C}$ NMR $\left(100 \mathrm{MHz}, \mathrm{CDCl}_{3}\right): \delta 167.0,142.5,133.5,129.5$, 128.7, 128.5, 128.5, 128.4, 125.8, 52.6, 35.9, 31.4, 27.2, 26.8; HRMS (ESI) $m / z:[\mathrm{M}+\mathrm{H}]^{+}$Calcd for $\mathrm{C}_{18} \mathrm{H}_{22} \mathrm{O}_{2} \mathrm{~N} 284.1645$; Found 284.1648.

\section{O-Benzoyl-N-(((1R,4aS,10aR)-7-isopropyl-1,4a-dimethyl-1,2,3,4,4a,9,10,10a-octahydrophenanthren-1-}

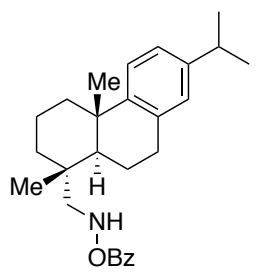

yl)methyl)hydroxylamine (1m): Prepared by the general procedure A from leelamine $(1.00 \mathrm{~g}, 3.51$ $\mathrm{mmol}$ ), purified by silica gel column chromatography (hexane/EtOAc), and isolated as a colorless oil (857 mg, 61\% yield). IR (thin film): $v$ 3247, 2957, 2929, 2867, 1720, 1451, 1268, 1087, $1066 \mathrm{~cm}^{-1}$; ${ }_{1}^{1} \mathrm{H}$ NMR (400 MHz, CDCl 3$): \delta$ 8.03-7.97 (m, 2H), 7.61-7.55 (m, 1H), 7.49-7.42 (m, 2H), $7.18(\mathrm{~d}, J=$ $8.1 \mathrm{~Hz}, 1 \mathrm{H}), 6.99(\mathrm{dd}, J=8.1,2.1 \mathrm{~Hz}, 1 \mathrm{H}), 6.88(\mathrm{~d}, J=2.1 \mathrm{~Hz}, 1 \mathrm{H}), 3.08-2.76(\mathrm{~m}, 5 \mathrm{H}), 2.36-2.26(\mathrm{~m}$, 1H), 1.96-1.56 (m, 7H), 1.51-1.39 (m, 1H), 1.26-1.16 (m, 8H), $1.06(\mathrm{~s}, 3 \mathrm{H}) ;{ }^{13} \mathrm{C}$ NMR (100 MHz, CDCl $): ~ \delta 167.3,147.3$, 145.7, 134.8, 133.5, 129.5, 128.7, 128.5, 126.9, 124.3, 124.0, 63.6, 46.2, 38.5, 37.7, 37.5, 36.8, 33.6, 30.2, 25.5, 24.2, 24.1, 19.3, 19.2, 18.9; HRMS (ESI) $m / z:[\mathrm{M}+\mathrm{H}]^{+}$Calcd for $\mathrm{C}_{27} \mathrm{H}_{36} \mathrm{O}_{2} \mathrm{~N}$ 406.2741; Found 406.2742; $\alpha \mathrm{D}^{28.9}=53.3$ (c 0.52, $\mathrm{CHCl}_{3}$ ).

tert-Butyl (benzoyloxy)(4-(thiophen-3-yl)butyl)carbamate (Boc-S1): Prepared by the general procedure B from 4-

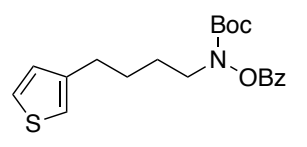

(thiophen-3-yl)butan-1-ol (234 mg, $1.50 \mathrm{mmol})$, purified by silica gel column chromatography (hexane/EtOAc), and isolated as a colorless oil (523 mg, 93\% yield). IR (thin film): $v$ 2977, 2935, 2862, 1764, 1714, 1452, 1368, 1243, 1156, 1100, 1055, $1012 \mathrm{~cm}^{-1} ;{ }^{1} \mathbf{H}$ NMR (400 MHz, $\left.\mathrm{CDCl}_{3}\right): \delta 8.09$ $8.02(\mathrm{~m}, 2 \mathrm{H}), 7.66-7.58(\mathrm{~m}, 1 \mathrm{H}), 7.52-7.43(\mathrm{~m}, 2 \mathrm{H}), 7.24-7.20(\mathrm{~m}, 1 \mathrm{H}), 6.95-6.90(\mathrm{~m}, 2 \mathrm{H}), 3.72(\mathrm{t}, J=6.6 \mathrm{~Hz}, 2 \mathrm{H}), 2.68(\mathrm{t}$, $J=7.1 \mathrm{~Hz}, 2 \mathrm{H}), 1.82-1.61(\mathrm{~m}, 4 \mathrm{H}), 1.46(\mathrm{~s}, 9 \mathrm{H}) ;{ }^{13} \mathrm{C}$ NMR $\left(100 \mathrm{MHz}, \mathrm{CDCl}_{3}\right): \delta$ 164.8, 155.0, 142.5, 133.9, 130.0, 128.7,

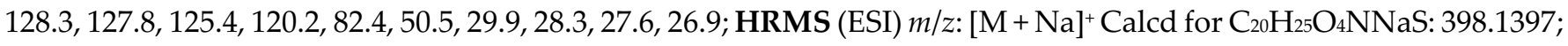
Found 398.1404.

O-Benzoyl-N-(4-(thiophen-3-yl)butyl)hydroxylamine (S1): Prepared by the general procedure C from tert-butyl

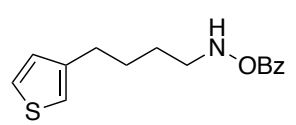
(benzoyloxy)(4-(thiophen-3-yl)butyl)carbamate $(407 \mathrm{mg}, 1.08 \mathrm{mmol})$, purified by silica gel column chromatography (hexane/EtOAc), and isolated as a colorless oil (255 mg, 86\% yield). IR (thin film): $v$ 3236, 2937, 2859, 1718, 1451, 1270, $1065 \mathrm{~cm}^{-1} ;{ }^{1} \mathbf{H}$ NMR $\left(400 \mathrm{MHz}, \mathrm{CDCl}_{3}\right): \delta$ 8.04-7.98 (m, 2H), 7.63-7.56 (m, 1H), 7.50-7.43 (m, 2H), 7.26-7.21 (m, 1H), 6.97-6.92 (m, 2H), 3.18 (t, J = 7.0 Hz, 2H), $2.69(\mathrm{t}, J=7.3 \mathrm{~Hz}, 2 \mathrm{H}), 1.84-1.64(\mathrm{~m}, 4 \mathrm{H}) ;{ }^{13} \mathrm{C}$ NMR (100 MHz, $\left.\mathrm{CDCl}_{3}\right): \delta 166.9,142.5,133.5,129.5,128.7,128.5,128.3$, 125.5, 120.3, 52.4, 30.11, 28.0, 26.8; HRMS (ESI) $m / z:[\mathrm{M}+\mathrm{H}]^{+}$Calcd for $\mathrm{C}_{15} \mathrm{H}_{18} \mathrm{O}_{2} \mathrm{NS}$ 276.1053; Found 276.1060.

O-Benzoyl-N-(4-(pyridin-4-yl)butyl)hydroxylamine (S2): Prepared by the general procedure A from 4-(pyridin-4-

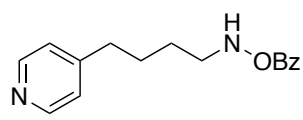

yl)butan-1-amine (317 mg, $2.11 \mathrm{mmol}$ ), purified by silica gel column chromatography (hexane/EtOAc), and isolated as a colorless oil (530 mg, 93\% yield). IR (thin film): $v$ 3235, 3068, 2940, 2862, 1721, 1602, 1451, 1416, 1271, 1090, 1066, $1024 \mathrm{~cm}^{-1}$; ${ }^{1} \mathbf{H}$ NMR (400 MHz, $\left.\mathrm{CDCl}_{3}\right): \delta$ 8.52-8.45 (m, 2H), 8.03-7.96 (m, 2H), $7.89(\mathrm{~s}, 1 \mathrm{H}), 7.62-7.55(\mathrm{~m}, 1 \mathrm{H}), 7.49-7.41(\mathrm{~m}, 2 \mathrm{H}), 7.14-7.08(\mathrm{~m}, 2 \mathrm{H}), 3.16(\mathrm{t}, J=$ Page S8 of S60 
$6.9 \mathrm{~Hz}, 2 \mathrm{H}), 2.66(\mathrm{t}, J=7.5 \mathrm{~Hz}, 2 \mathrm{H}), 1.84-1.73(\mathrm{~m}, 2 \mathrm{H}), 1.73-1.61(\mathrm{~m}, 2 \mathrm{H}) ;{ }^{13} \mathrm{C}$ NMR $(100 \mathrm{MHz}, \mathrm{CDCl}): \delta$ 167.0, 151.4, 149.6, 133.5, 129.4, 128.7, 128.5, 124.1, 52.3, 35.1, 27.7, 26.9; HRMS (ESI) $m / z:[\mathrm{M}+\mathrm{H}]^{+}$Calcd for $\mathrm{C}_{16} \mathrm{H}_{19} \mathrm{O}_{2} \mathrm{~N}_{2} 271.1441$; Found 271.1448.

tert-Butyl (benzoyloxy)(3-( $p$-tolyl)propyl)carbamate (Boc-3): Prepared by the general procedure B from 3-(pBoc tolyl)propan-1-ol (321 mg, $2.12 \mathrm{mmol})$, purified by silica gel column chromatography (hexane/EtOAc), and isolated as a colorless oil (642 mg, 82\% yield). IR (thin film): $v 2977,2931$, 1765, 1714, 1452, 1368, 1242, 1158, 1090, 1050, $1010 \mathrm{~cm}^{-1}$; ${ }^{1} \mathrm{H}$ NMR (400 MHz, CDCl$): ~ \delta 8.11-8.04(\mathrm{~m}, 2 \mathrm{H}), 7.67-7.58$ $(\mathrm{m}, 1 \mathrm{H}), 7.52-7.45(\mathrm{~m}, 2 \mathrm{H}), 7.12-7.06(\mathrm{~m}, 4 \mathrm{H}), 3.73(\mathrm{t}, J=7.0 \mathrm{~Hz}, 2 \mathrm{H}), 2.69(\mathrm{t}, J=7.3 \mathrm{~Hz}, 2 \mathrm{H}), 2.31(\mathrm{~s}, 3 \mathrm{H}), 2.02-1.90(\mathrm{~m}$, 2H), 1.47 (s, 9H); ${ }^{13} \mathrm{C}$ NMR (100 MHz, $\left.\mathrm{CDCl}_{3}\right): \delta 164.8,155.0,138.5,135.5,133.9,130.0,129.2,128.7,128.5,127.8,82.5$, 50.4, 32.6, 29.3, 28.3, 21.1; HRMS (ESI) $m / z$ : [M + Na $]^{+}$Calcd for $\mathrm{C}_{22} \mathrm{H}_{27} \mathrm{O}_{4} \mathrm{NNa}$ 392.1832; Found 392.1837.

$O$-Benzoyl-N-(3-(p-tolyl)propyl)hydroxylamine (3): Prepared by the general procedure C from tert-butyl (benzoyloxy)(3-(p-tolyl)propyl)carbamate (200 mg, $0.54 \mathrm{mmol})$, purified by silica gel column chromatography (hexane/EtOAc), and isolated as a colorless oil (136 mg, 94\% yield). IR (thin film): $v$ 3237, 2942, 2860, 1719, 1514, 1451, 1269, 1091, 1065, $1024 \mathrm{~cm}^{-1} ;{ }^{1} \mathbf{H}$ NMR (400 MHz, CDCl $): \delta 8.05-7.99(\mathrm{~m}, 2 \mathrm{H})$, 7.62-7.56 (m, 1H), 7.50-7.42 (m, 2H), 7.12-7.09 (m, 4H), 3.22-3.12 (m, 2H), 2.75-2.68 (m, 2H), $2.32(\mathrm{~s}, 3 \mathrm{H}), 2.01-1.91$ (m, 2H); ${ }^{13} \mathrm{C}$ NMR (100 MHz, $\left.\mathrm{CDCl}_{3}\right): \delta$ 167.0, 138.4, 135.6, 133.5, 129.5, 129.3, 128.7, 128.5, 128.4, 52.1, 32.9, 29.0, 21.1; HRMS (ESI) $m / z:[\mathrm{M}+\mathrm{Na}]^{+}$Calcd for $\mathrm{C}_{17} \mathrm{H}_{19} \mathrm{O}_{2} \mathrm{NNa} 292.1308$; Found 292.1312.

tert-Butyl (benzoyloxy)(4-(4-fluorophenyl)butyl-4- $d$ )carbamate (Boc-5): Prepared by the general procedure B from 4-(4-fluorophenyl)butan-4- $d-1-\mathrm{ol}^{3}$ (310 mg, $\left.1.83 \mathrm{mmol}\right)$, purified by silica gel column
chromatography (hexane/EtOAc), and isolated as a colorless oil (682 mg, 96\% yield). IR (thin $1013 \mathrm{~cm}^{-1} ;{ }^{1} \mathrm{H}$ NMR $\left(400 \mathrm{MHz}, \mathrm{CDCl}_{3}\right): \delta 8.08-8.01(\mathrm{~m}, 2 \mathrm{H}), 7.66-7.58(\mathrm{~m}, 1 \mathrm{H}), 7.52-7.44(\mathrm{~m}, 2 \mathrm{H}), 7.16-7.06(\mathrm{~m}, 2 \mathrm{H})$, 6.98-6.88 (m, 2H), $3.71(\mathrm{t}, J=6.5 \mathrm{~Hz}, 2 \mathrm{H}), 2.60(\mathrm{t}, J=7.0 \mathrm{~Hz}, 1 \mathrm{H}), 1.74-1.62(\mathrm{~m}, 4 \mathrm{H}), 1.45(\mathrm{~s}, 9 \mathrm{H}) ;{ }^{13} \mathrm{C} \mathrm{NMR}(100 \mathrm{MHz}$, $\left.\mathrm{CDCl}_{3}\right): \delta 164.8,161.4(\mathrm{~d}, J=243.2 \mathrm{~Hz}), 155.1,137.8(\mathrm{~d}, J=3.1 \mathrm{~Hz}), 133.9,130.0,129.8(\mathrm{~d}, J=7.7 \mathrm{~Hz}), 128.8,127.8,115.1$ $(\mathrm{d}, J=21.0 \mathrm{~Hz}), 82.5,50.5,34.3(\mathrm{t}, J=19.2 \mathrm{~Hz}), 28.5,28.3,26.7 ;{ }^{19} \mathrm{~F} \mathrm{NMR}\left(376 \mathrm{MHz}, \mathrm{CDCl}_{3}\right) \delta-117.91$; HRMS (ESI) $\mathrm{m} / z$ : $[\mathrm{M}+\mathrm{Na}]^{+}$Calcd for $\mathrm{C}_{22} \mathrm{H}_{25} \mathrm{DO}_{4} \mathrm{NFNa}$ 411.1801; Found 411.1804.

$O$-Benzoyl-N-(4-(4-fluorophenyl)butyl-4- $d$ )hydroxylamine (5): Prepared by the general procedure C from tert-butyl (1) (benzoyloxy)(4-(4-fluorophenyl)butyl-4- $d$ )carbamate $(600 \mathrm{mg}, 1.54 \mathrm{mmol})$, purified by silica gel column chromatography (hexane/EtOAc), and isolated as a colorless oil (439 mg, 99\% yield). IR (thin film): $v$ 3236, 3065, 3037, 2935, 2862, 1719, 1601, 1509, 1452, 1270, 1219, 1092, 1066, $1025 \mathrm{~cm}^{-1}$; ${ }^{1} \mathrm{H}$ NMR (400 MHz, $\left.\mathrm{CDCl}_{3}\right): \delta$ 8.04-7.95 (m, 2H), 7.64-7.56 (m, 1H), 7.50-7.41 (m, 2H), 7.18-7.09 (m, 2H), 6.99-6.90 (m, 2H), $6.75(\mathrm{~s}, 1 \mathrm{H}), 3.16(\mathrm{t}, J=6.8 \mathrm{~Hz}, 2 \mathrm{H}), 2.61(\mathrm{t}, J=7.4 \mathrm{~Hz}, 1 \mathrm{H}), 1.80-1.59(\mathrm{~m}, 4 \mathrm{H}) ;{ }^{13} \mathrm{C}$ NMR $(100$ $\left.\mathrm{MHz}, \mathrm{CDCl}_{3}\right): \delta 166.9,161.4(\mathrm{~d}, J=243.3 \mathrm{~Hz}), 137.7(\mathrm{~d}, J=3.2 \mathrm{~Hz}), 133.5,129.8$ (d, J = $\left.7.7 \mathrm{~Hz}\right), 129.5,128.7,128.4,115.2$ 
$(\mathrm{d}, J=21.0 \mathrm{~Hz}), 52.4,34.5(\mathrm{t}, J=19.6 \mathrm{~Hz}), 28.9,26.7 ;{ }^{19} \mathrm{~F} \mathbf{N M R}\left(376 \mathrm{MHz}, \mathrm{CDCl}_{3}\right) \delta-117.81 ; \mathrm{HRMS}(\mathrm{ESI}) \mathrm{m} / z:[\mathrm{M}+\mathrm{H}]^{+}$ Calcd for $\mathrm{C}_{17} \mathrm{H}_{18} \mathrm{DO}_{2} \mathrm{NF} 289.1457$; Found 289.1463.

tert-Butyl (benzoyloxy)(4-cyclopropylbutyl)carbamate (Boc-7): Prepared by the general procedure B from 4-

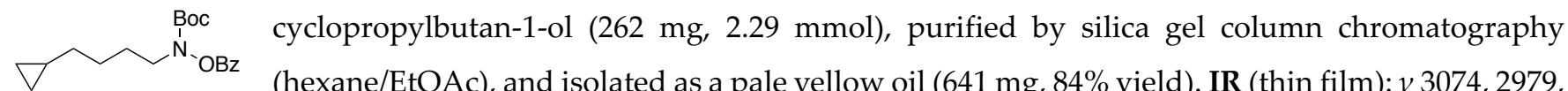
2932, 2857, 1766, 1714, 1452, 1392, 1368, 1244, 1158, $1014 \mathrm{~cm}^{-1}$; ${ }^{1} \mathrm{H}$ NMR (400 MHz, CDCl $): \delta 8.11-8.05$ (m, 2H), 7.66$7.58(\mathrm{~m}, 2 \mathrm{H}), 7.51-7.44(\mathrm{~m}, 2 \mathrm{H}), 3.69(\mathrm{t}, J=7.2 \mathrm{~Hz}, 2 \mathrm{H}), 1.76-1.61(\mathrm{~m}, 2 \mathrm{H}), 1.52-1.41(\mathrm{~m}, 11 \mathrm{H}), 1.27-1.17(\mathrm{~m}, 2 \mathrm{H}), 0.72-$ $0.59(\mathrm{~m}, 1 \mathrm{H}), 0.44-0.34(\mathrm{~m}, 2 \mathrm{H}), 0.04-0.06(\mathrm{~m}, 2 \mathrm{H}) ;{ }^{13} \mathrm{C}$ NMR $\left(100 \mathrm{MHz}, \mathrm{CDCl}_{3}\right): \delta 164.8,155.1,133.9,130.0,128.7$, $127.9,82.4,50.9,34.4,28.3,27.2,26.8,10.9,4.5$; HRMS (ESI) $m / z$ : $[\mathrm{M}+\mathrm{Na}]^{+}$Calcd for $\mathrm{C}_{19} \mathrm{H}_{27} \mathrm{O}_{4} \mathrm{NNa} 356.1832$; Found 356.1836.

O-Benzoyl- $N$-(4-cyclopropylbutyl)hydroxylamine (7): Prepared by the general procedure C from tert-butyl

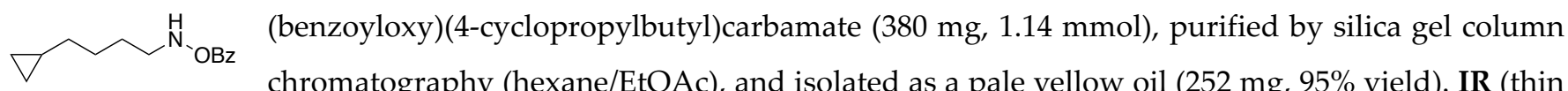
film): $v$ 3237, 3073, 2998, 2932, 2856, 1719, 1451, 1271, 1089, 1066, $1024 \mathrm{~cm}^{-1} ;{ }^{1} \mathbf{H}$ NMR $\left(400 \mathrm{MHz}, \mathrm{CDCl}_{3}\right): \delta 8.06-7.99$ $(\mathrm{m}, 2 \mathrm{H}), 7.63-7.55(\mathrm{~m}, 1 \mathrm{H}), 7.50-7.42(\mathrm{~m}, 2 \mathrm{H}), 3.16(\mathrm{t}, J=6.7 \mathrm{~Hz}, 2 \mathrm{H}), 1.75-1.61(\mathrm{~m}, 2 \mathrm{H}), 1.57-1.46(\mathrm{~m}, 2 \mathrm{H}), 1.30-1.20$ $(\mathrm{m}, 2 \mathrm{H}), 0.73-0.60(\mathrm{~m}, 1 \mathrm{H}), 0.45-0.35(\mathrm{~m}, 2 \mathrm{H}), 0.05-0.03(\mathrm{~m}, 2 \mathrm{H}) ;{ }^{13} \mathrm{C}$ NMR $\left(100 \mathrm{MHz}, \mathrm{CDCl}_{3}\right): \delta 166.9,133.5,129.5$, 128.7, 128.5, 52.7, 34.6, 27.2, 27.0, 10.9, 4.5; HRMS (ESI) $m / z:[\mathrm{M}+\mathrm{Na}]^{+}$Calcd for $\mathrm{C}_{14} \mathrm{H}_{19} \mathrm{O}_{2} \mathrm{NNa} 256.1308$; Found 256.1313. 


\section{Rh-catalyzed $\mathrm{C}-\mathrm{H}$ functionalization}

\section{3-1. Initial screenings}

\section{3-1-1. Screenings with halogenating agents.}

Table S1. Conditions screening with halogenating agent

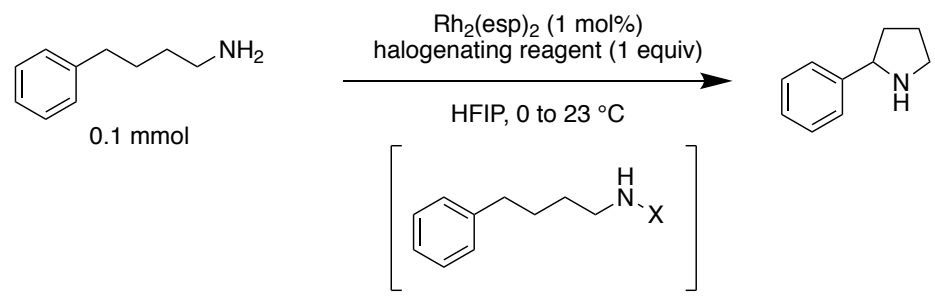

\begin{tabular}{|c|c|}
\hline entry & halogenating reagent \\
\hline 1 & NCS \\
\hline 2 & $\mathbf{A}$ \\
\hline 3 & B \\
\hline 4 & NBS \\
\hline 5 & NIS \\
\hline 6 & $\mathrm{I}_{2}$ \\
\hline & $\underset{\mathrm{O}}{\mathcal{C}_{\mathrm{Cl}}^{\mathrm{Cl}}} \underset{\mathrm{A}}{\mathrm{Cl}}=\mathrm{O}$ \\
\hline
\end{tabular}

Table S2. Conditions screening with prepared $\mathrm{N}-\mathrm{Cl}$ amine

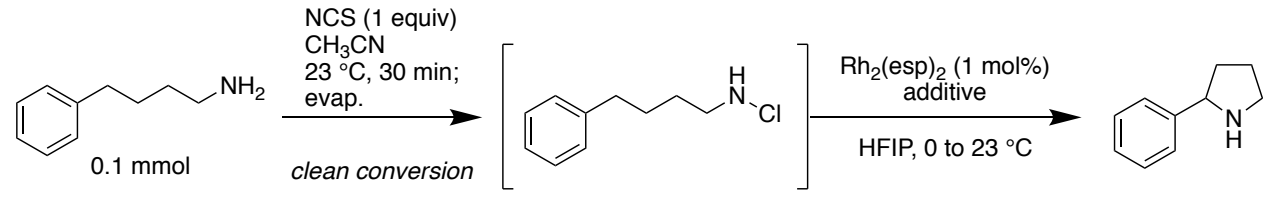

$\begin{array}{lll}\text { entry } & \text { additive } & \text { yield (\%) } \\ 1 & \text { None } & 0 \\ 2 & \mathrm{~K}_{2} \mathrm{CO}_{3}(1 \text { equiv) } & \mathrm{NR} \\ 3 & \mathrm{Et}_{3} \mathrm{~N}(1 \text { equiv) } & \mathrm{NR} \\ 4 & \mathrm{DBU}(1 \text { equiv) } & \mathrm{NR} \\ 5 & \mathrm{AcOH}(1 \text { equiv) } & \text { complex mixture } \\ 6 & \mathrm{TFA}(1 \text { equiv) } & \text { complex mixture } \\ 7 & \mathrm{HCl} / \mathrm{EtOAc}(1 \text { equiv) } & \text { complex mixture } \\ 8 & \mathrm{TsOH} \bullet \mathrm{H}_{2} \mathrm{O}(1 \text { equiv) } & \text { complex mixture }\end{array}$




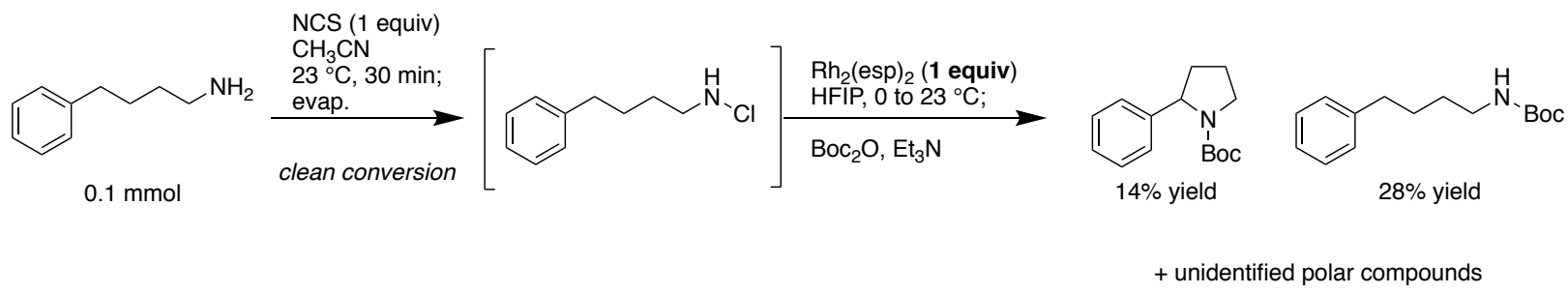

The stoichiometric Rh experiment suggested that the desired cyclization occurred, but it was hard to control, one of the major side reactions was a reduction of the chloroamine by a cleavage of the $\mathrm{N}-\mathrm{Cl}$ bond.

\section{3-1-2. Solvent Screening with $O$-Bz hydroxylamine.}

Table S3. Solvent screening with O-Bz hydroxylamine 1a.

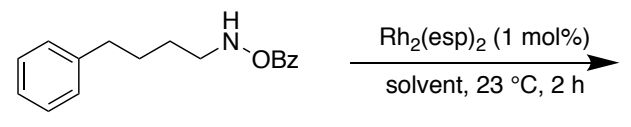

$1 \mathbf{a}$

$$
\text { entry }
$$

1

2

3

4

5

\section{3-2. Substrate scope for pyrrolidine synthesis (Figure 1)}

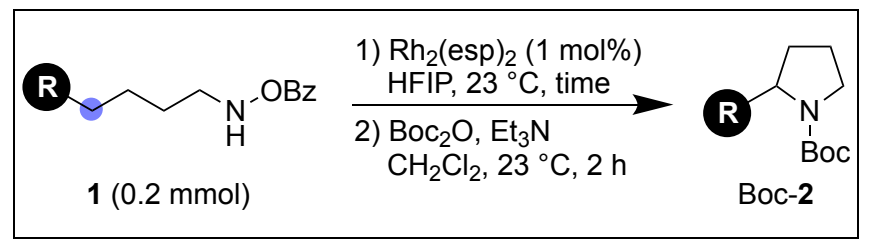

General procedure D: To a $10 \mathrm{~mL}$ test tube equipped with a magnetic stirring bar were added $\mathrm{O}$ benzoylhydroxylamines 1 ( $0.2 \mathrm{mmol})$, HFIP ( $2 \mathrm{~mL}, 0.1 \mathrm{M})$, followed by the addition of $\mathrm{Rh} 2(\mathrm{esp}) 2(1 \mathrm{~mol} \%, 0.002$ $\mathrm{mmol})$ at $23{ }^{\circ} \mathrm{C}$. The resulting mixture was stirred at the same temperature. After full consumption of the substrates by TLC analysis, the reaction mixture was concentrated under reduced pressure. To the crude residue were added $\mathrm{CH}_{2} \mathrm{Cl}_{2}(1 \mathrm{ml}, 0.2 \mathrm{M}), \mathrm{Et} 3 \mathrm{~N}$ (2.0 equiv), and $\mathrm{Boc}_{2} \mathrm{O}$ (2.0 equiv). The solution was stirred for $2 \mathrm{~h}$ at $23^{\circ} \mathrm{C}$ and directly placed on a silica gel column chromatography, affording the N-heterocycles.

$\mathrm{N}$-Boc 2-phenylpyrrolidine (Boc-2a): Prepared by the general procedure D from O-benzoyl- $N$-(4phenylbutyl)hydroxylamine 1a $(53.9 \mathrm{mg}, 0.2 \mathrm{mmol})$, purified by silica gel column chromatography 1 Boc (hexane/EtOAc), and isolated as a colorless oil (43.5 mg, 88\% yield). ${ }^{1} \mathbf{H} ~ \mathbf{N M R}\left(400 \mathrm{MHz}, \mathrm{CDCl}_{3}\right.$, rotamer ratio 30:70): $\delta 7.33-7.24(\mathrm{~m}, 2 \mathrm{H}), 7.24-7.19(\mathrm{~m}, 1 \mathrm{H}), 7.19-7.12(\mathrm{~m}, 2 \mathrm{H}), 4.95$ (brs, 0.3H), 4.76 (brs, 0.7H), 3.70-3.48 (m, $2 \mathrm{H}), 2.40-2.20(\mathrm{~m}, 1 \mathrm{H}), 2.00-1.77(\mathrm{~m}, 3 \mathrm{H}), 1.45(\mathrm{~s}, 2.7 \mathrm{H}), 1.18(\mathrm{~s}, 6.3 \mathrm{H})$. Spectroscopic data matched those reported. ${ }^{4}$ 
$N$-Boc 2-( $p$-tolyl)pyrrolidine (Boc-2b): Prepared by the general procedure D from O-benzoyl- $N-(4-(p-$

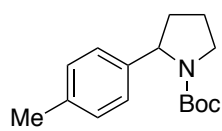
tolyl)butyl)hydroxylamine $\mathbf{1 b}(56.7 \mathrm{mg}, 0.2 \mathrm{mmol})$, purified by silica gel column chromatography (hexane/EtOAc), and isolated as a colorless oil $\left(47.0 \mathrm{mg}, 90 \%\right.$ yield). ${ }^{1} \mathbf{H}$ NMR (400 MHz, $\mathrm{CDCl}_{3}$, rotamer ratio 30:70): $\delta 7.09(\mathrm{~d}, J=7.9 \mathrm{~Hz}, 2 \mathrm{H}), 7.04(\mathrm{~d}, J=7.9 \mathrm{~Hz}, 2 \mathrm{H}), 4.90$ (brs, 0.3H), 4.75 (brs, 0.7H), 3.67-3.45 (m, 2H), 2.39-2.20 (m, 4H), 2.01-1.74 (m, 3H), $1.44(\mathrm{~s}, 2.7 \mathrm{H}), 1.20(\mathrm{~s}, 6.3 \mathrm{H})$. Spectroscopic data matched those reported. ${ }^{5}$

$\mathrm{N}$-Boc 2-(4-methoxyphenyl)pyrrolidine (Boc-2c): Prepared by the general procedure D from O-benzoyl- $\mathrm{N}-(4-(4-$

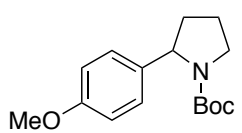
methoxyphenyl)butyl)hydroxylamine $1 c(59.9 \mathrm{mg}, 0.2 \mathrm{mmol})$, purified by silica gel column chromatography (hexane/EtOAc), and isolated as a colorless oil (48.2 mg, $87 \%$ yield). ${ }^{1} \mathbf{H}$ NMR (400 MHz, $\mathrm{CDCl}_{3}$, rotamer ratio 30:70): $\delta 7.08(\mathrm{~d}, J=8.1 \mathrm{~Hz}, 2 \mathrm{H}), 6.83(\mathrm{~d}, J=8.1 \mathrm{~Hz}, 2 \mathrm{H}), 4.90$ (brs, $0.3 \mathrm{H}), 4.73(\mathrm{brs}, 0.7 \mathrm{H}), 3.79(\mathrm{~s}, 3 \mathrm{H}), 3.70-3.45(\mathrm{~m}, 2 \mathrm{H}), 2.35-2.20(\mathrm{~m}, 1 \mathrm{H}), 1.96-1.74(\mathrm{~m}, 3 \mathrm{H}), 1.44(\mathrm{~s}, 2.7 \mathrm{H}), 1.20(\mathrm{~s}, 6.3 \mathrm{H})$. Spectroscopic data matched those reported. ${ }^{5}$

N-Boc 4-(pyrrolidin-2-yl)benzonitrile (Boc-2d): Prepared by the general procedure D from 4-(4-

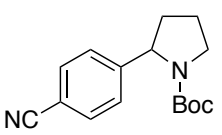
((benzoyloxy)amino)butyl)benzonitrile $1 \mathbf{d}(58.9 \mathrm{mg}, 0.2 \mathrm{mmol})$, purified by silica gel column chromatography (hexane/EtOAc), and isolated as a colorless oil (48.4 mg, $89 \%$ yield). ${ }^{1} \mathbf{H}$ NMR $\left(400 \mathrm{MHz}, \mathrm{CDCl}_{3}\right.$, rotamer ratio 30:70): $\delta 7.60(\mathrm{~d}, J=8.1 \mathrm{~Hz}, 2 \mathrm{H}), 7.35-7.23(\mathrm{~m}, 2 \mathrm{H}), 4.94(\mathrm{brs}, 0.3 \mathrm{H})$, 4.79 (brs, 0.7H), 3.71-3.51 (m, 2H), 2.45-2.27 (m, 1H), 1.97-1.73 (m, 3H), 1.44 (s, 2.7H), 1.18 (s, 6.3H). Spectroscopic data matched those reported. ${ }^{4}$

$\mathrm{N}$-Boc methyl 4-(pyrrolidin-2-yl)benzoate (Boc-2e): Prepared by the general procedure D from methyl 4-(4-

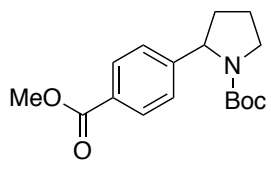
((benzoyloxy)amino)butyl)benzoate $1 \mathrm{e}(65.5 \mathrm{mg}, 0.2 \mathrm{mmol})$, purified by silica gel column chromatography (toluene/EtOAc), and isolated as a colorless oil (54.9 mg, 90\% yield). ${ }^{1} \mathbf{H}$ NMR (400 MHz, $\mathrm{CDCl}_{3}$, rotamer ratio 30:70): $\delta$ 8.02-7.94 $(\mathrm{m}, 2 \mathrm{H}), 7.30-7.20(\mathrm{~m}, 2 \mathrm{H}), 4.97(\mathrm{brs}, 0.3 \mathrm{H})$, 4.80 (brs, 0.7H), 3.91 (s, 3H), 3.71-3.48 (m, 2H), 2.45-2.23 (m, 1H), 1.95-1.72 (m, 3H), $1.45(\mathrm{~s}, 2.7 \mathrm{H}), 1.17(\mathrm{~s}, 6.3 \mathrm{H})$. Spectroscopic data matched those reported. ${ }^{4}$

$\mathrm{N}$-Boc 2-(3-methoxyphenyl)pyrrolidine (Boc-2f): Prepared by the general procedure D from O-benzoyl- $\mathrm{N}-(4-(3-$

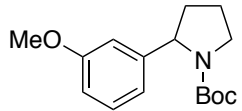
methoxyphenyl)butyl)hydroxylamine If $(59.9 \mathrm{mg}, 0.2 \mathrm{mmol})$, purified by silica gel column chromatography (hexane/EtOAc), and isolated as a colorless oil (52.1 mg, 94\% yield). ${ }^{1} \mathbf{H}$ NMR (400 MHz, $\mathrm{CDCl}_{3}$, rotamer ratio 30:70): $\delta 7.21(\mathrm{dd}, J=7.9,7.9 \mathrm{~Hz}, 1 \mathrm{H}), 6.80-6.73(\mathrm{~m}, 2 \mathrm{H}), 6.72-6.69$ (m, 1H), 4.93 (brs, 0.3H), 4.74 (brs, 0.7H), $3.79(\mathrm{~s}, 3 \mathrm{H}), 3.68-3.50(\mathrm{~m}, 2 \mathrm{H}), 2.36-2.20(\mathrm{~m}, 1 \mathrm{H}), 1.99-1.77(\mathrm{~m}, 3 \mathrm{H}), 1.46(\mathrm{~s}$, $2.7 \mathrm{H}), 1.20(\mathrm{~s}, 6.3 \mathrm{H})$. Spectroscopic data matched those reported. ${ }^{6}$ 
$\mathrm{N}$-Boc methyl 2-(pyrrolidin-2-yl)benzoate (Boc-2g): Prepared by the general procedure D from methyl 2-(4-

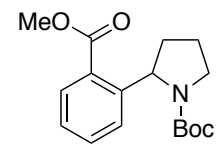

((benzoyloxy)amino)butyl)benzoate $1 \mathrm{~g}(65.5 \mathrm{mg}, 0.2 \mathrm{mmol})$, purified by silica gel column chromatography (hexane/EtOAc), and isolated as a colorless oil ( $53.7 \mathrm{mg}, 88 \%$ yield). ${ }^{1} \mathrm{H}$ NMR (400 $\mathrm{MHz}, \mathrm{CDCl}_{3}$, rotamer ratio 30:70): $\delta$ 7.98-7.92 $(\mathrm{m}, 0.3 \mathrm{H}), 7.92-7.84(\mathrm{~m}, 0.7 \mathrm{H}), 7.46(\mathrm{td}, J=7.6,1.5 \mathrm{~Hz}$,

1H), 7.36-7.21 (m, 2H), 5.70 (brs, 0.3H), 5.51 (brs, 0.7H), 3.97-3.84 (m, 3H), 3.70-3.60 (m, 1.4H), 3.58-3.50 (m, 0.6H), 2.58-2.36 (m, 1H), 1.95-1.71 (m, 3H), $1.45(\mathrm{~s}, 2.7 \mathrm{H}), 1.13(\mathrm{~s}, 6.3 \mathrm{H})$. Spectroscopic data matched those reported. ${ }^{7}$

$N$-Boc 2,2,5-trimethylpyrrolidine (Boc-2i): Prepared by the general procedure D from O-benzoyl- $N$-(5-methylhexan2-yl)hydroxylamine 1i $(47.1 \mathrm{mg}, 0.2 \mathrm{mmol})$, purified by silica gel column chromatography Boc (hexane/EtOAc), and isolated as a colorless oil (35.8 mg, 84\% yield). IR (thin film): $v$ 2982, 2938, 1809, 1771, 1758, 1373, 1213, 1119, $1069 \mathrm{~cm}^{-1}$; 1 H NMR (400 MHz, CDCl $)$ : $\delta 3.98$ (brs, 1H), 2.01-1.87 (m, 2H), 1.73-1.66 (m, 1H), $1.57(\mathrm{~s}, 3 \mathrm{H}), 1.46(\mathrm{~s}, 9 \mathrm{H}), 1.48-1.36(\mathrm{~m}, 1 \mathrm{H}), 1.28(\mathrm{~s}, 3 \mathrm{H}), 1.15(\mathrm{~d}, J=6.4 \mathrm{~Hz}, 3 \mathrm{H}) ;{ }^{13} \mathrm{C}$ NMR $\left(100 \mathrm{MHz}, \mathrm{CDCl}_{3}\right): \delta$ 154.4, 78.9, 60.8, 55.3, 40.3, 29.1, 28.9, 27.6, 25.7, 21.0; HRMS (ESI) $m / z$ : $[\mathrm{M}+\mathrm{H}]^{+}$Calcd for $\mathrm{C}_{12} \mathrm{H}_{24} \mathrm{O}_{2} \mathrm{~N}$ 214.1803; Found 214.1802.

$N$-Boc 2-propylpyrrolidine (Boc-2j): Prepared by the general procedure D from O-benzoyl- $N$-heptylhydroxylamine

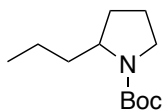

$\mathbf{1 j}$ (47.1 mg, $0.2 \mathrm{mmol}$ ), purified by silica gel column chromatography (hexane/EtOAc), and isolated $\underset{\text { Boc }}{N}$ as a colorless oil (29.8 mg, 70\% yield). IR (thin film): $v 2964,2932,2873,1696,1393,1172,1103 \mathrm{~cm}^{-1} ;{ }^{1} \mathbf{H}$ NMR (400 MHz, CDCl $): \delta 3.80-3.65(\mathrm{~m}, 1 \mathrm{H}), 3.39-3.23(\mathrm{~m}, 2 \mathrm{H}), 1.97-1.71(\mathrm{~m}, 3 \mathrm{H}), 1.70-1.55(\mathrm{~m}, 2 \mathrm{H}), 1.45(\mathrm{~s}, 9 \mathrm{H})$, 1.36-1.00 (m, 3H), $0.91(\mathrm{t}, J=7.2 \mathrm{~Hz}, 3 \mathrm{H}) ;{ }^{13} \mathrm{C}$ NMR (100 MHz, $\left.\mathrm{CDCl}_{3}\right): \delta 154.9,79.1,57.3,46.3,36.8,30.4,28.7,23.5$, 19.7, 14.3; HRMS (ESI) m/z: [M + Na $]^{+}$Calcd for $\mathrm{C}_{12} \mathrm{H}_{23} \mathrm{O}_{2} \mathrm{NNa}$ 236.1621; Found 236.1626.

$\mathrm{N}$-Boc 2-benzylpyrrolidine (Boc-2k): Prepared by the general procedure D from O-benzoyl- $N$-(5-

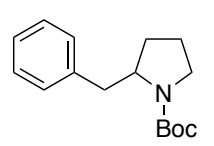
phenylpentyl)hydroxylamine 1k (56.7 mg, $0.2 \mathrm{mmol})$, purified by silica gel column chromatography (hexane/EtOAc), and isolated as a colorless oil (38.6 mg, 74\% yield). ${ }^{1} \mathbf{H}$ NMR $\left(400 \mathrm{MHz}, \mathrm{CDCl}_{3}\right): \delta$ 7.33-7.24 (m, 2H), 7.24-7.12 (m, 3H), 4.07-3.88 (m, 1H), 3.45-3.25 (m, 2H), 3.20-2.95 (m, 1H), 2.65-2.48 (m, 1H), 1.82-1.65 (m, 4H), $1.51(\mathrm{~s}, 9 \mathrm{H})$. Spectroscopic data matched those reported. ${ }^{8}$

$N$-Boc (3aR, 3a $\left.{ }^{1} R, 5 a S, 10 b S\right)-8$-isopropyl-3a,10b-dimethyl-1,2,3,3a,3a ${ }^{1}, 4,5,5 a, 6,10 b$-decahydrodibenzo[ $[c d, f]$ indole

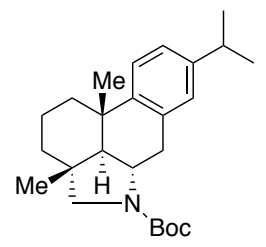

(Boc-2m): Prepared by the general procedure D from O-benzoyl- $N-(((1 R, 4 a S, 10 a R)-7-$ isopropyl1,4a-dimethyl-1,2,3,4,4a,9,10,10a-octahydrophenanthren-1-yl)methyl)hydroxylamine 1m $(81.1 \mathrm{mg}$, $0.2 \mathrm{mmol}$ ), purified by silica gel column chromatography (hexane/EtOAc), and isolated as a colorless oil (65.9 mg, 86\% yield). ${ }^{1} \mathbf{H}$ NMR (400 MHz, $\mathrm{CDCl}_{3}$, rotamer ratio 35:65): $\delta 7.14$ (t, J = 7.5 $\mathrm{Hz}, 1 \mathrm{H}), 7.08-7.00(\mathrm{~m}, 1 \mathrm{H}), 6.98-6.91(\mathrm{~m}, 1 \mathrm{H}), 3.88-3.51(\mathrm{~m}, 2 \mathrm{H}), 3.49$ (d, J = $10.0 \mathrm{~Hz}, 0.65 \mathrm{H}), 3.37$ $(\mathrm{d}, J=10.4 \mathrm{~Hz}, 0.35 \mathrm{H}), 2.99-2.91(\mathrm{~m}, 1 \mathrm{H}), 2.90-2.78(\mathrm{~m}, 1 \mathrm{H}), 2.76-2.64(\mathrm{~m}, 1 \mathrm{H}), 2.24-2.14(\mathrm{~m}, 1 \mathrm{H}), 1.94-1.68(\mathrm{~m}, 3 \mathrm{H})$, 1.61-1.46 (m, 10H), 1.43-1.30 (m, 1H), 1.27-1.15 (m, 10H), $1.09(\mathrm{~s}, 3 \mathrm{H})$. Spectroscopic data matched those reported.$^{8}$ 


\section{3-3. Pyrrolidine synthesis on a larger scale (eq 1)}

To a $100 \mathrm{~mL}$ flask tube equipped with a magnetic stirring bar were added O-benzoylhydroxylamines $\mathbf{1 a}$ (1.35 $\mathrm{g}$, $5.00 \mathrm{mmol})$, HFIP $(25.0 \mathrm{~mL}, 0.2 \mathrm{M})$, followed by the addition of Rh2(esp)2 $(3.79 \mathrm{mg}, 0.1 \mathrm{~mol} \%, 5.00 \mu \mathrm{mol})$ at $23{ }^{\circ} \mathrm{C}$. The resulting mixture was stirred at the same temperature for $3 \mathrm{~h}$, during which time 1a was fully consumed. The reaction mixture was concentrated under reduced pressure. To the crude residue were added $\mathrm{CH}_{2} \mathrm{Cl}_{2}$ (25.0 ml, $\left.0.2 \mathrm{M}\right), \mathrm{Et}_{3} \mathrm{~N}$ (1.67 mL, 2.4 equiv), and $\mathrm{Boc} 2 \mathrm{O}(1.30 \mathrm{~g}, 1.2$ equiv). The solution was stirred for 2 $h$ at $23{ }^{\circ} \mathrm{C}$. After the addition of $\mathrm{H}_{2} \mathrm{O}$, the aqueous phase was extracted with $\mathrm{CH}_{2} \mathrm{Cl}_{2}(3 \mathrm{x})$. The combined organic layers were wahsed with brine, dried over $\mathrm{Na}_{2} \mathrm{SO}_{4}$, filtered, and removed under reduced pressure. The obtained crude materials was purified by a silica gel column chromatography, with hexane/EtOAc (9:1) as the eluent, affording Boc-2a as a colorless oil (1.11 g, 90\% yield).

\section{3-4. List of unsuccessful substrates}

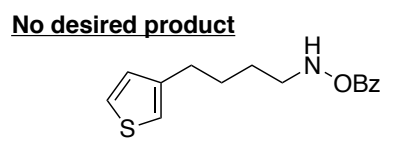

S1

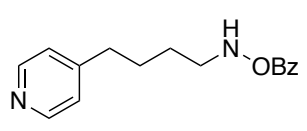

S2

Low product yield

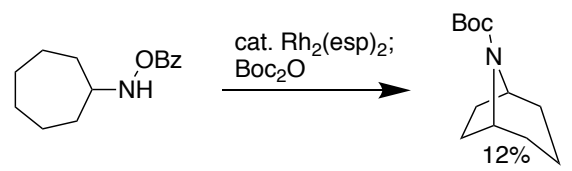

\section{3-5. Synthesis of tetrahydroquinoline 4 (Scheme 2)}

7-Methyl-1,2,3,4-tetrahydroquinoline (4): Prepared by the general procedure D from O-benzoyl- $N-(3-(p-$<smiles>Ic1ccc2c(c1)NCCC2</smiles>
tolyl)propyl)hydroxylamine $3(53.9 \mathrm{mg}, 0.2 \mathrm{mmol})$. After the consumption of the substrate, HFIP was removed under reduced pressure. Instead of the addition of $\mathrm{Boc}_{2} \mathrm{O}$, the crude material was purified by silica gel column chromatography (hexane/EtOAc), affording the title compound as a colorless oil (20.9 $\mathrm{mg}, 71 \%$ yield). ${ }^{1} \mathrm{H}$ NMR $\left(400 \mathrm{MHz}, \mathrm{CDCl}_{3}\right): \delta 6.84(\mathrm{~d}, J=7.6 \mathrm{~Hz}, 1 \mathrm{H}), 6.44(\mathrm{dd}, J=7.6,1.6 \mathrm{~Hz}, 1 \mathrm{H}), 6.32(\mathrm{~d}, J=1.6 \mathrm{~Hz}, 1 \mathrm{H})$, 3.35-3.24 (m, 2H), $2.73(\mathrm{t}, J=6.4 \mathrm{~Hz}, 2 \mathrm{H}), 2.22(\mathrm{~s}, 3 \mathrm{H}), 1.99-1.88(\mathrm{~m}, 2 \mathrm{H})$. Spectroscopic data matched those reported. ${ }^{9}$

\section{3-6. Mechanistic investigations (Scheme 3)}
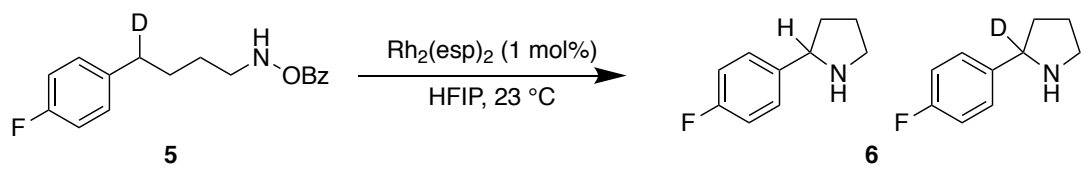

$20: 80$

By following General Procedure D, the reaction was conducted with $5(0.2 \mathrm{mmol})$. After the consumption of the substrate, the volatile was removed under reduced pressure. The crude material was analyzed by ${ }^{1} \mathrm{H}$ NMR to determine the kinetic isotope effect value. 


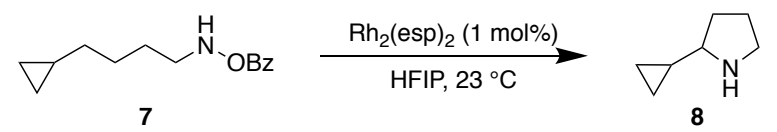

By following General Procedure D, the reaction was conducted with 7 (0.2 mmol). After the consumption of the substrate, the volatile was removed under reduced pressure. The crude material was analyzed by ${ }^{1} \mathrm{H}$ NMR, which indicates that no cyclopropyl opening occurred during the course of the transformation (the spectra is shown below).
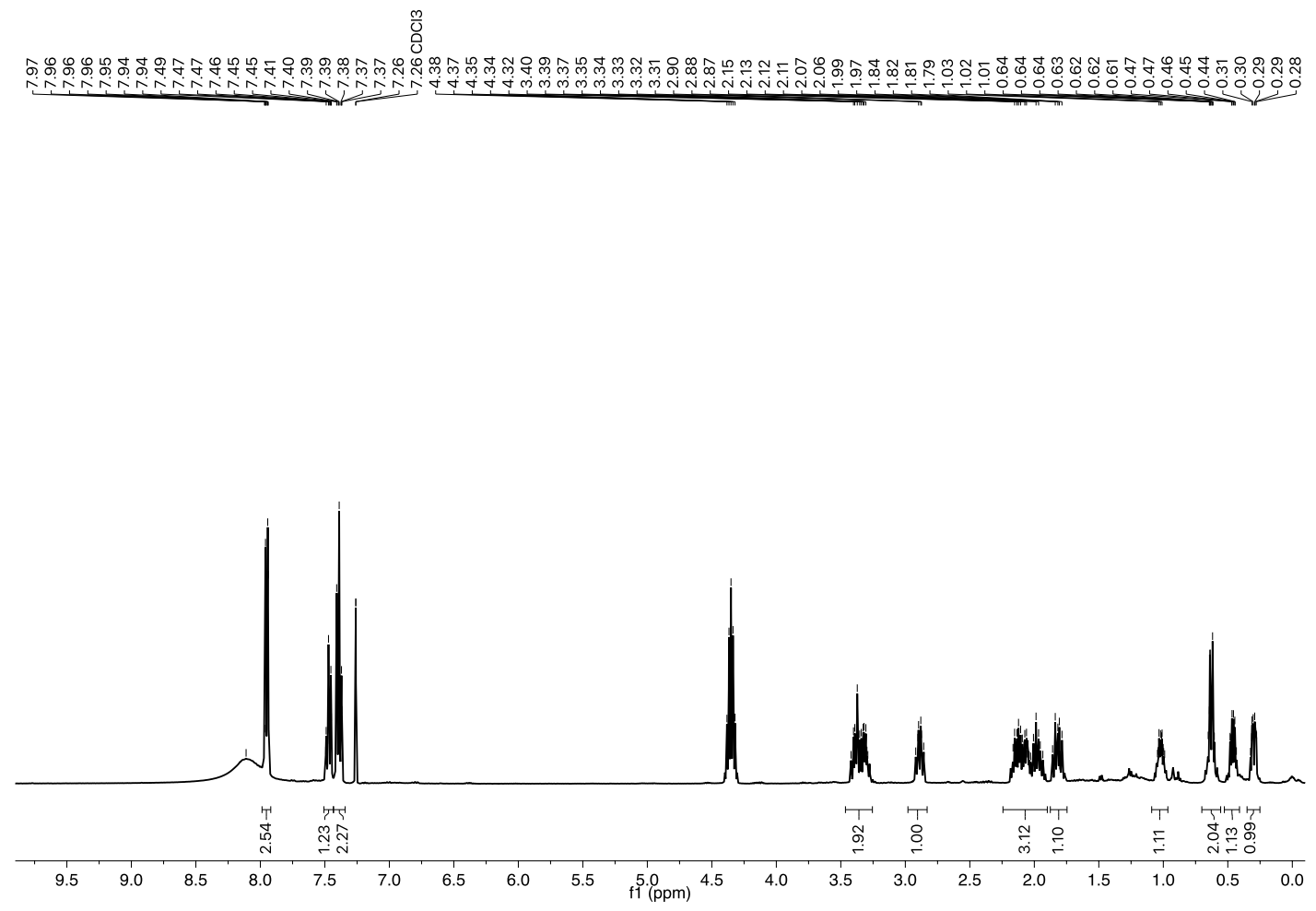


\section{References}

(1) Fulmer, G. R.; Miller, A. J. M.; Sherden, N. H.; Gottlieb, H. E.; Nudelman, A.; Stoltz, B. M.; Bercaw, J. E.; Goldberg, K. I. Organometallics 2010, 29, 2176.

(2) Banerjee, A.; Yamamoto, H. Chem. Sci. 2019, 10, 2124.

(3) Munnuri, S.; Adebesin, A. M.; Paudyal, M. P.; Yousufuddin, M.; Dalipe, A.; Falck, J. R. J. Am. Chem. Soc. 2017, 139, 18288.

(4) Campos, K. R.; Klapars, A.; Waldman, J. H.; Dormer, P. G.; Chen, C.-y. J. Am. Chem. Soc. 2006, 128, 11, 3538.

(5) Dieter, R. K.; Li, S. J. Org. Chem. 1997, 62, 7726.

(6) Chen, F.; Ding, Z.; Qin, J.; Wang, T.; He, Y.; Fan, Q.-H. Org. Lett. 2011, 13, 4348.

(7) Barker, G.; McGrath, J. L.; Klapars, A.; Stead, D.; Zhou, G.; Campos, K. R; O’Brien, P. J. Org. Chem. 2011, 76, 5936

(8) Shing, K.-P.; Liu, Y.; Cao, B.; Chang, X.-Y.; You, T.; Che, C.-M. Angew. Chem. Int. Ed. 2018, 57, 11947.

(9) WSS: Wiley Subscription Servies on SciFinder 


\section{NMR Spectra}

$O$-Benzoyl- $N$-(4-phenylbutyl)hydroxylamine (1a):

${ }^{1} \mathbf{H}$ NMR (400 MHz, $\left.\mathrm{CDCl}_{3}\right)$
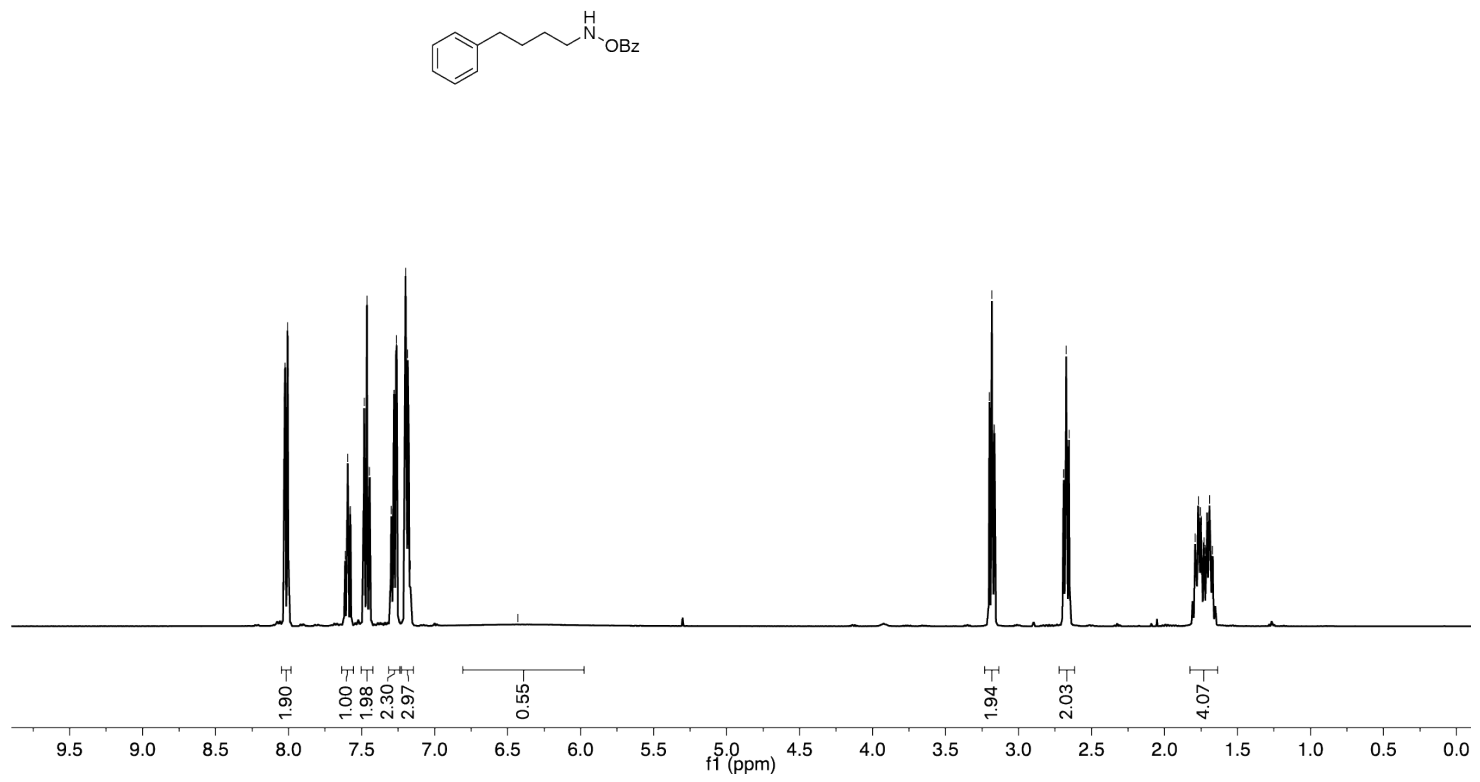

${ }^{13} \mathrm{C}$ NMR $\left(100 \mathrm{MHz}, \mathrm{CDCl}_{3}\right)$

\begin{tabular}{|c|c|c|c|c|c|}
\hline$\stackrel{\circ}{\circ}$ & 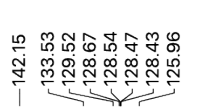 & 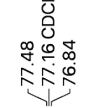 & 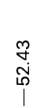 & 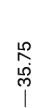 & 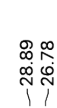 \\
\hline
\end{tabular}

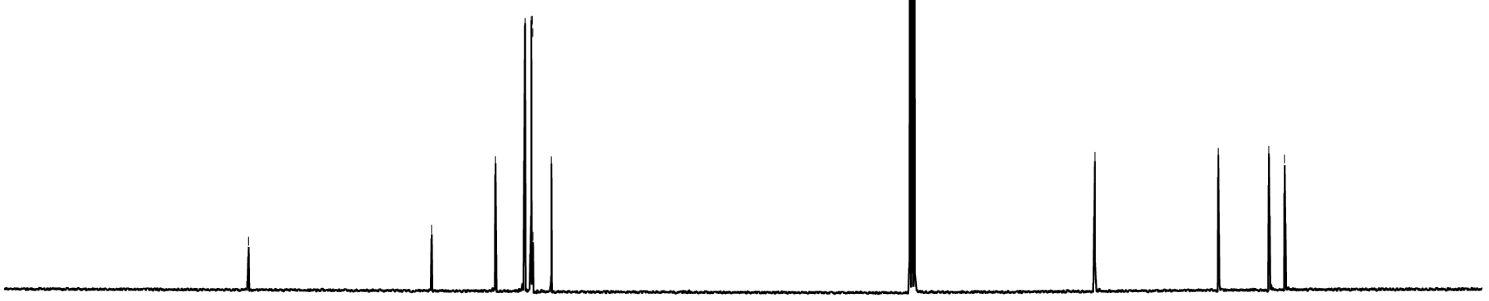

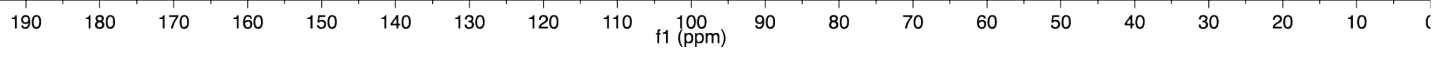

Page S18 of S60 
tert-Butyl (benzoyloxy)(4-( $p$-tolyl)butyl)carbamate (Boc-1b):

${ }^{1} \mathbf{H}$ NMR $\left(400 \mathrm{MHz}, \mathrm{CDCl}_{3}\right)$
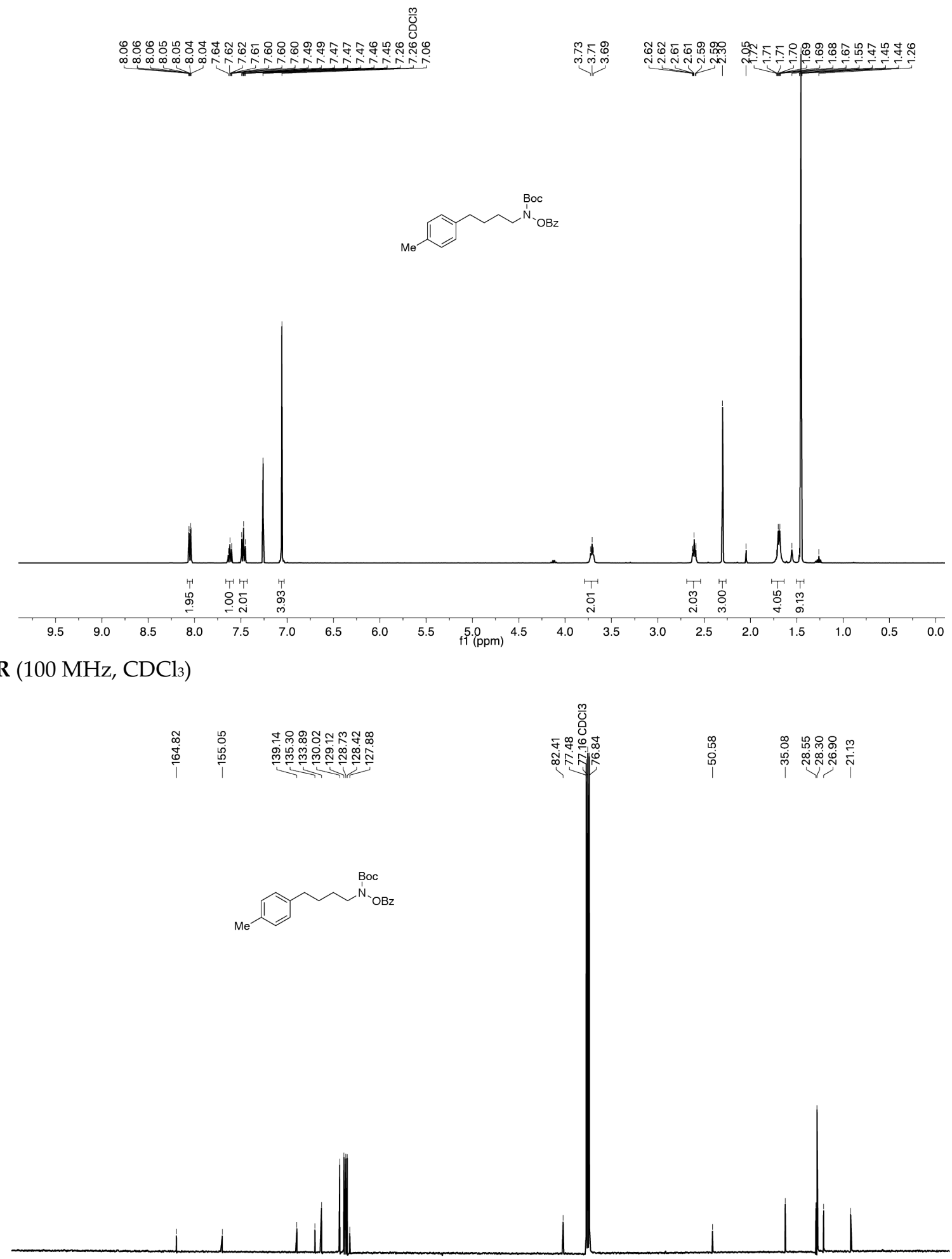

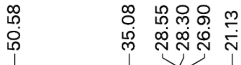

${ }^{13} \mathrm{C}$ NMR (100 MHz, $\left.\mathrm{CDCl}_{3}\right)$

190

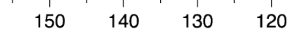

$110 \underset{f 1}{100}(\mathrm{ppm}) \quad 90$

80

Page S19 of S60 
O-Benzoyl-N-(4-( $p$-tolyl)butyl)hydroxylamine (1b):

${ }^{1} \mathbf{H}$ NMR (400 MHz, $\left.\mathrm{CDCl}_{3}\right)$
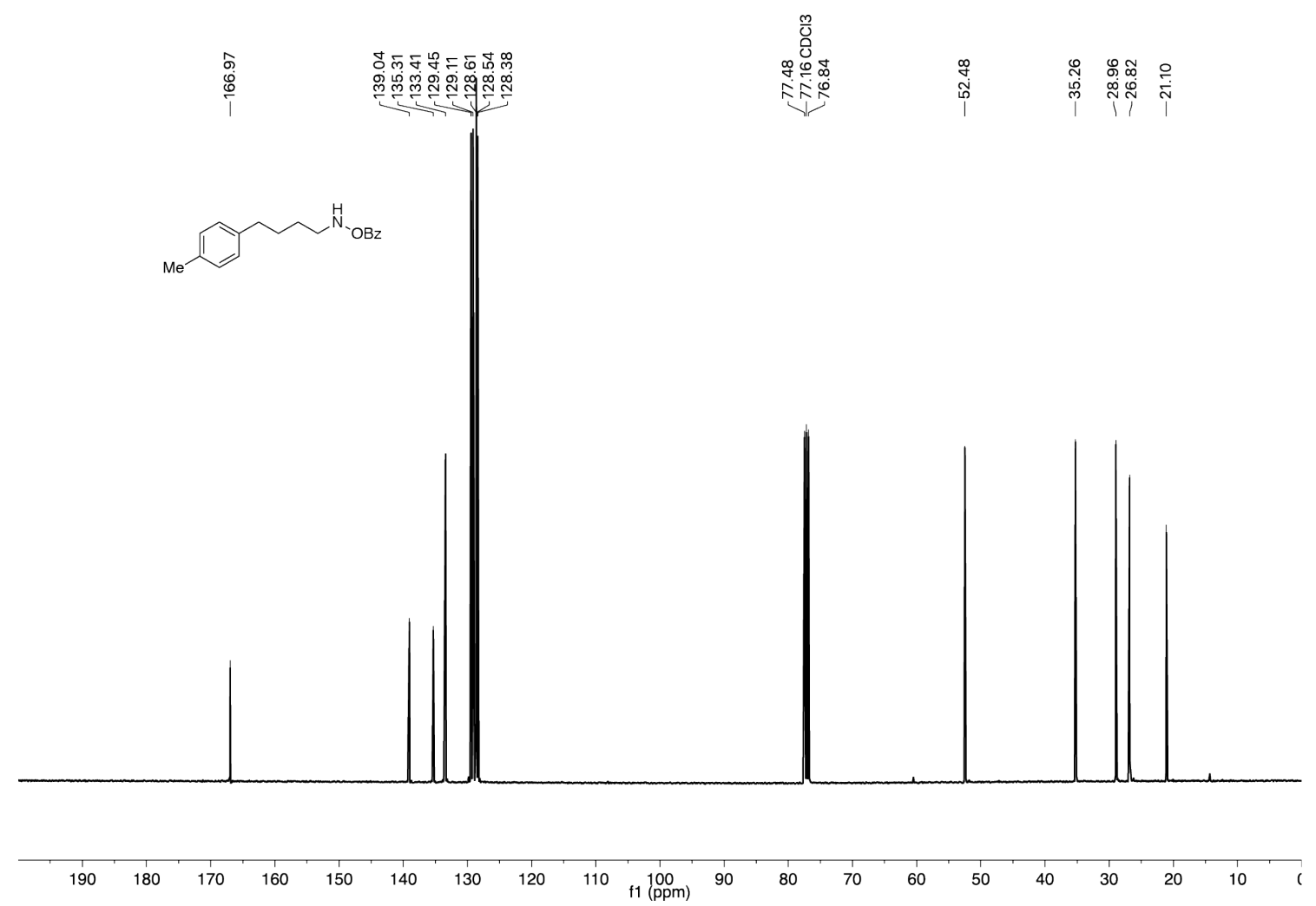

${ }^{13} \mathrm{C}$ NMR (100 MHz, $\left.\mathrm{CDCl}_{3}\right)$

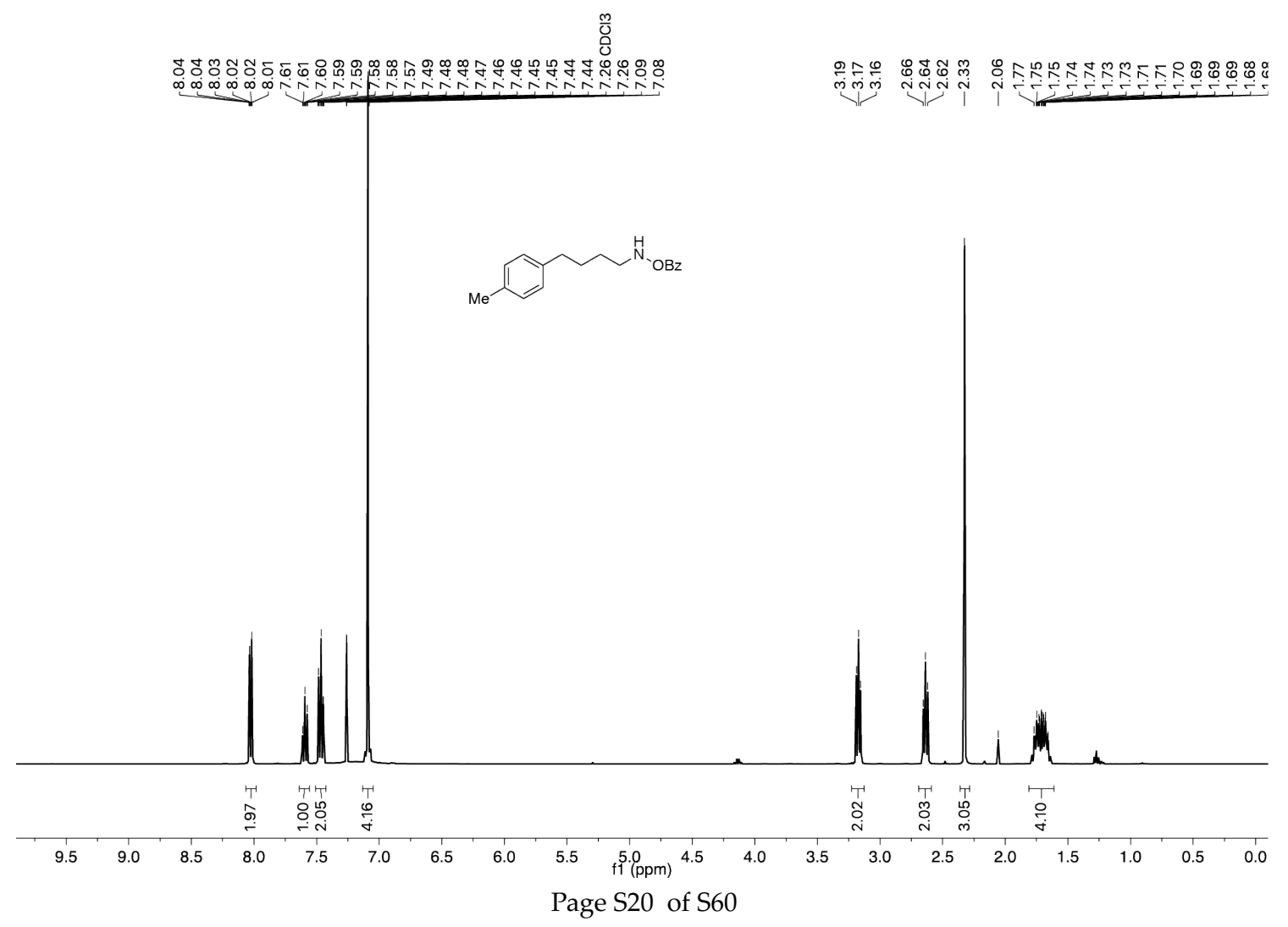


tert-Butyl (benzoyloxy)(4-(4-methoxyphenyl)butyl)carbamate (Boc-1c):

${ }^{1} \mathbf{H}$ NMR $\left(400 \mathrm{MHz}, \mathrm{CDCl}_{3}\right)$
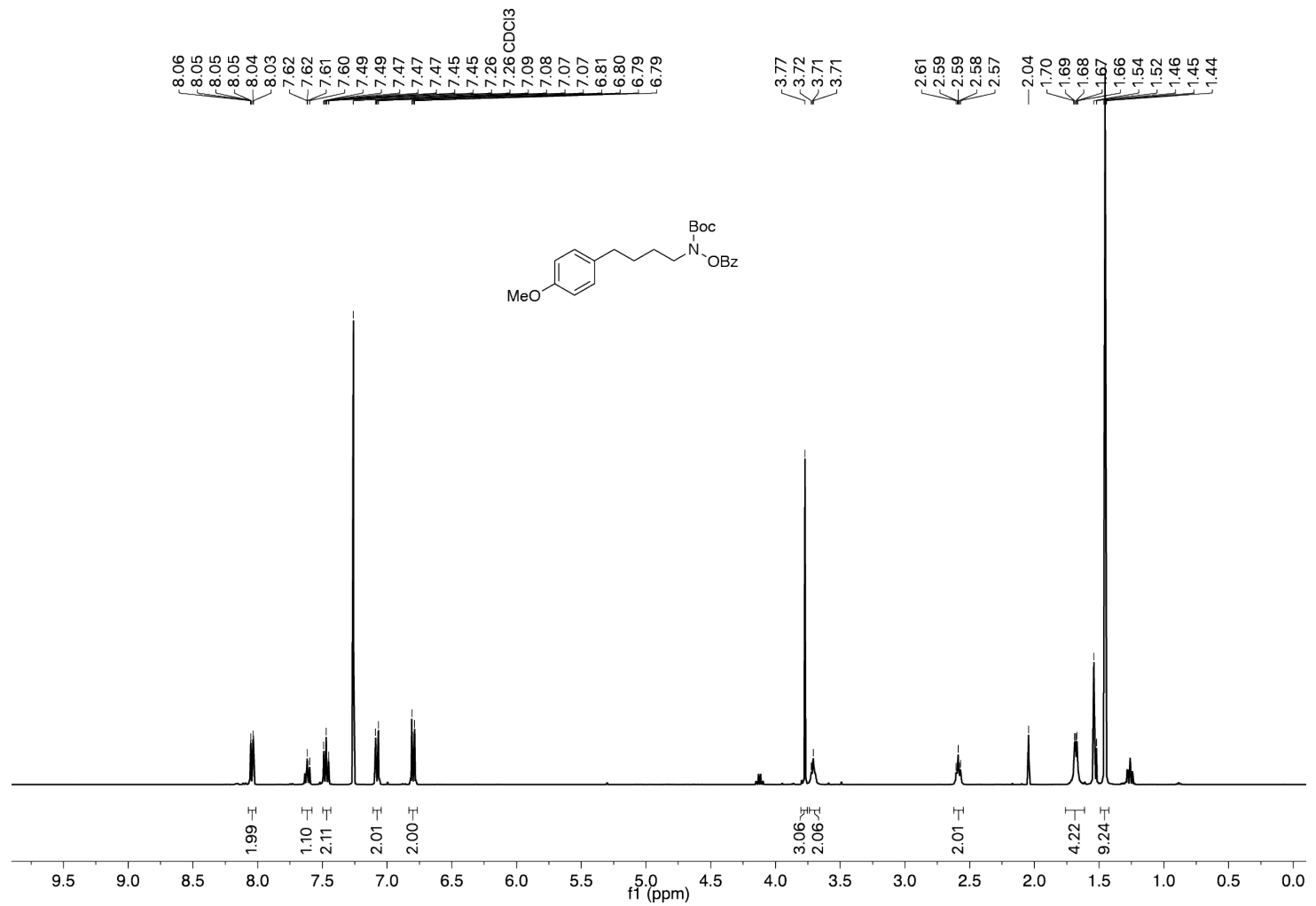

${ }^{13} \mathrm{C}$ NMR (100 MHz, $\left.\mathrm{CDCl}_{3}\right)$

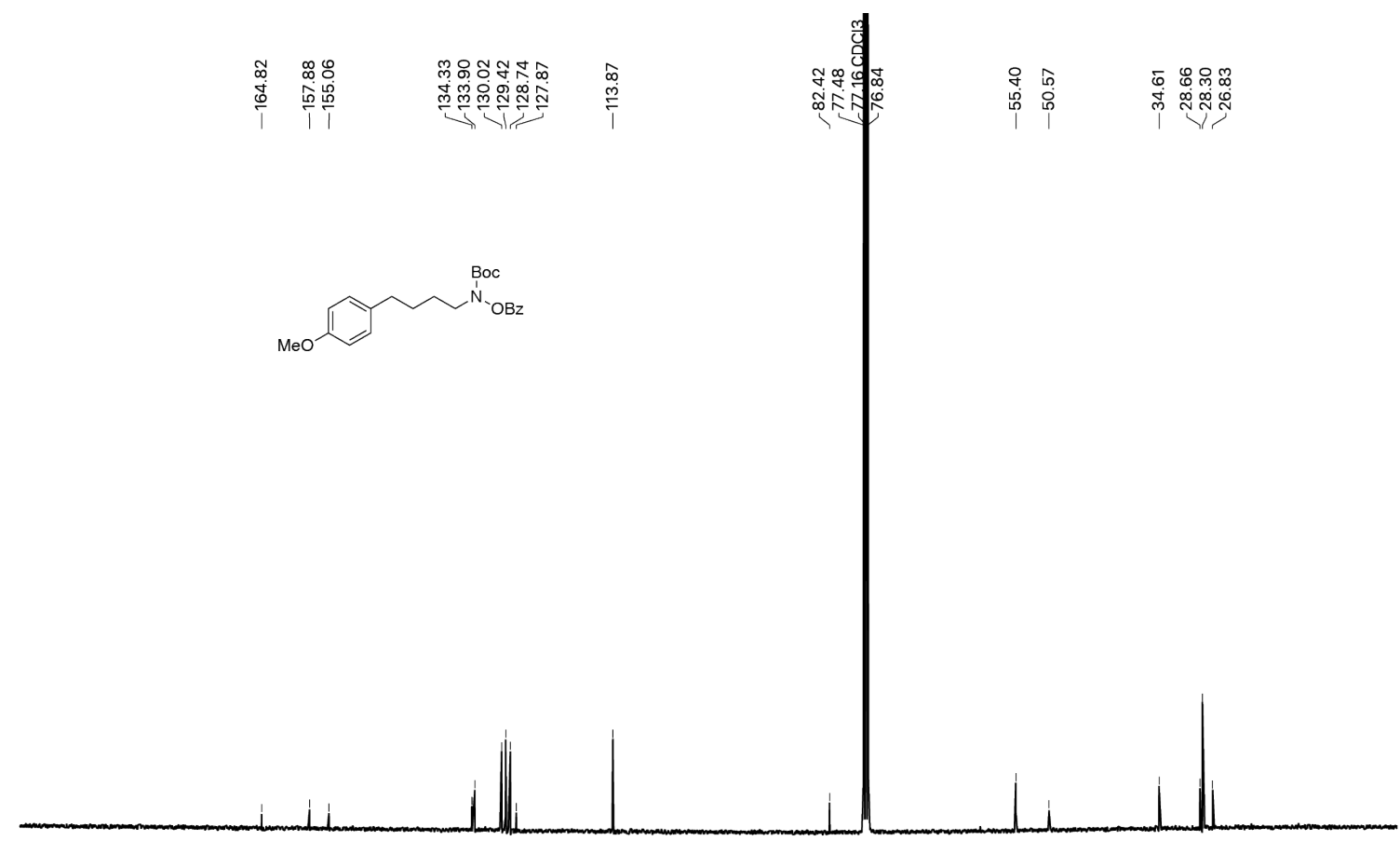

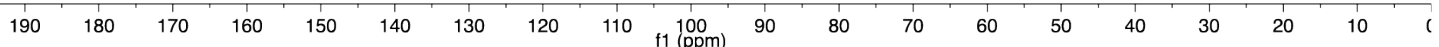

Page S21 of S60 
O-Benzoyl-N-(4-(4-methoxyphenyl)butyl)hydroxylamine (1c):

${ }^{1} \mathbf{H}$ NMR (400 MHz, $\left.\mathrm{CDCl}_{3}\right)$
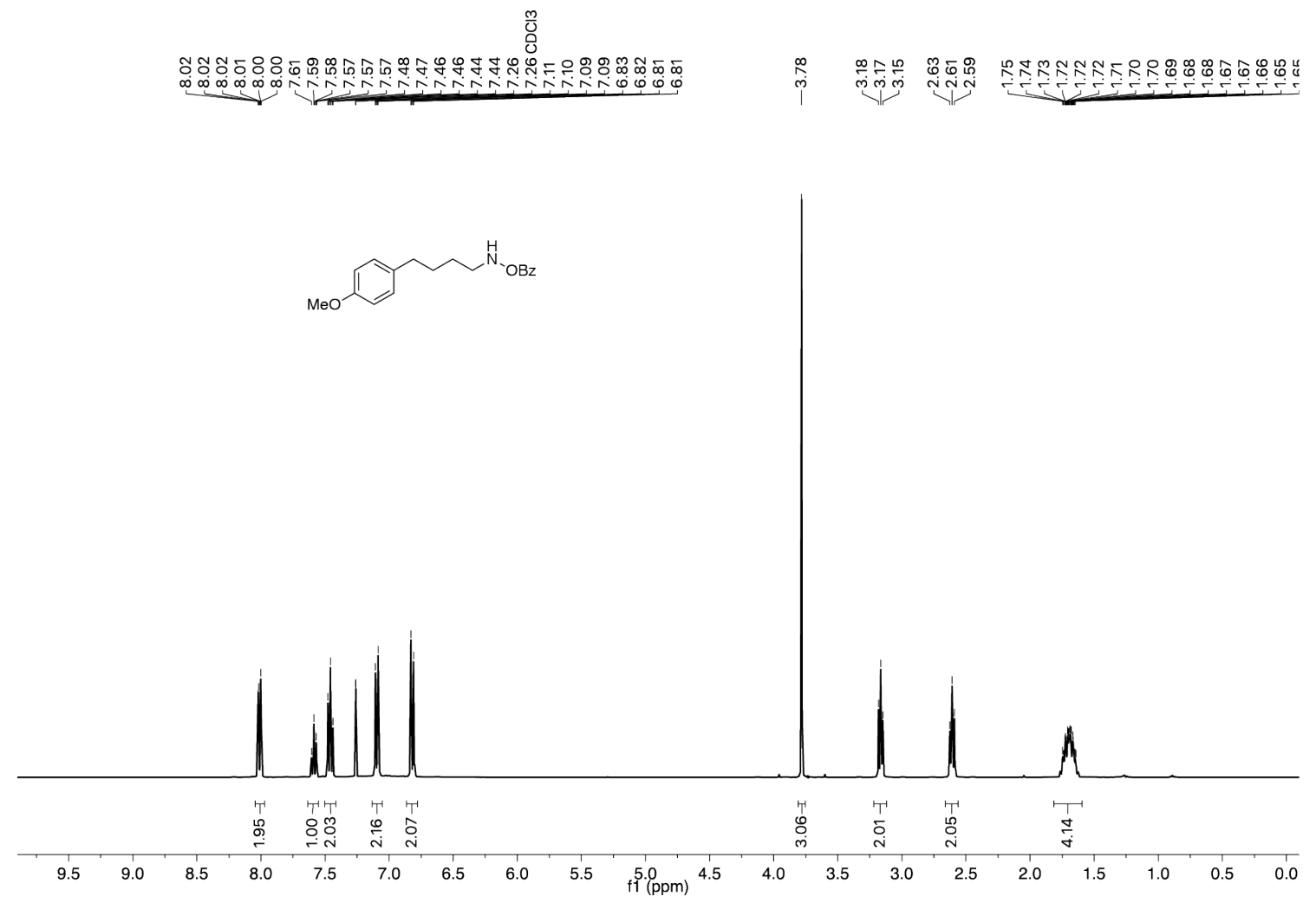

${ }^{13} \mathrm{C}$ NMR (100 MHz, $\left.\mathrm{CDCl}_{3}\right)$

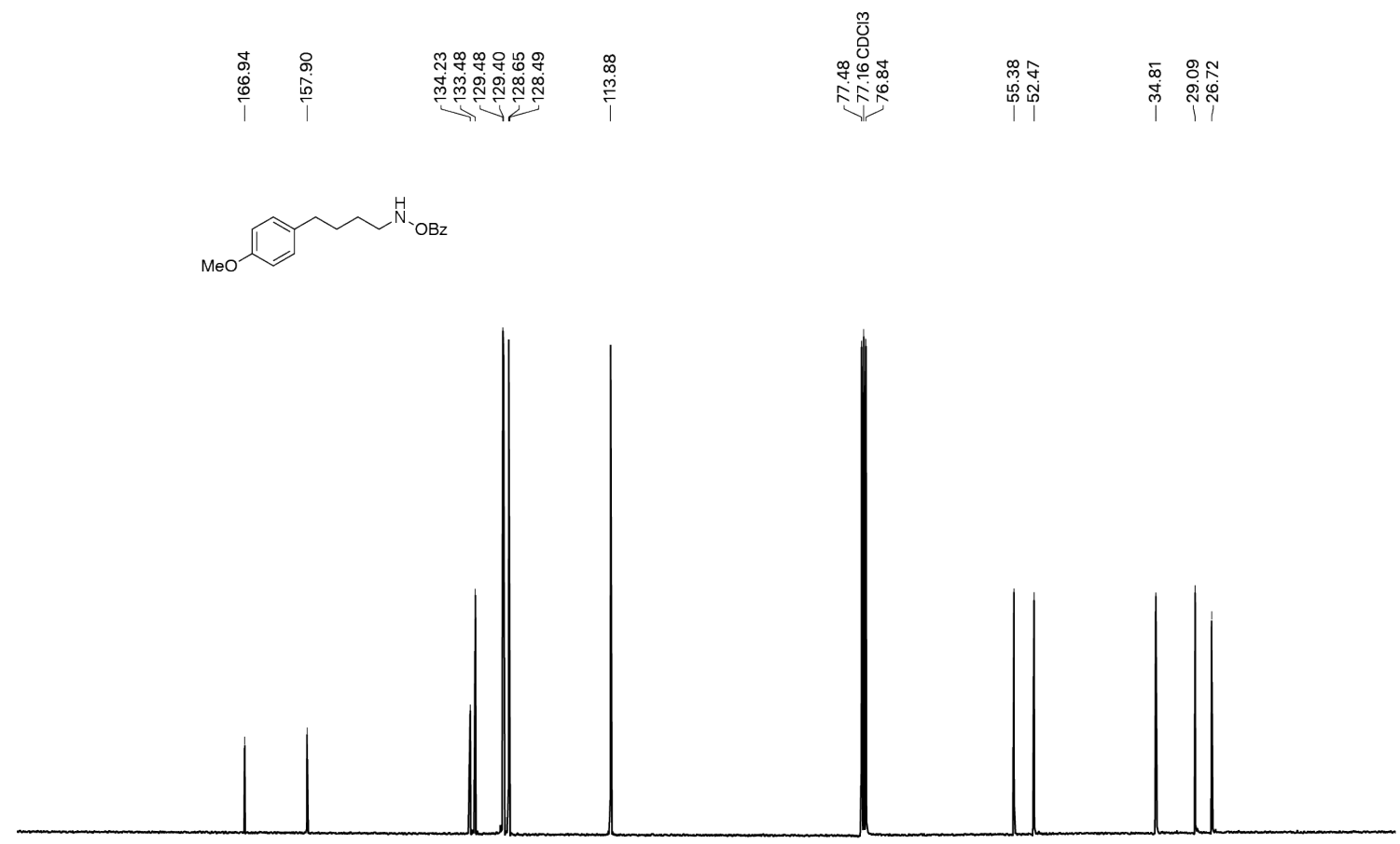

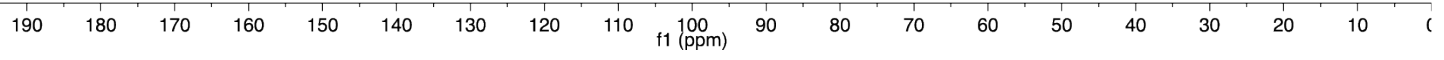

Page S22 of S60 
tert-Butyl (benzoyloxy)(4-(4-cyanophenyl)butyl)carbamate (Boc-1d):

${ }^{1} \mathbf{H}$ NMR (400 MHz, $\left.\mathrm{CDCl}_{3}\right)$

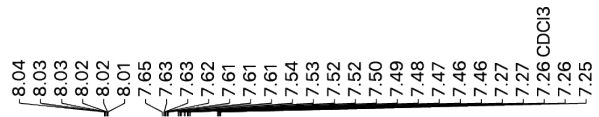

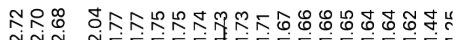
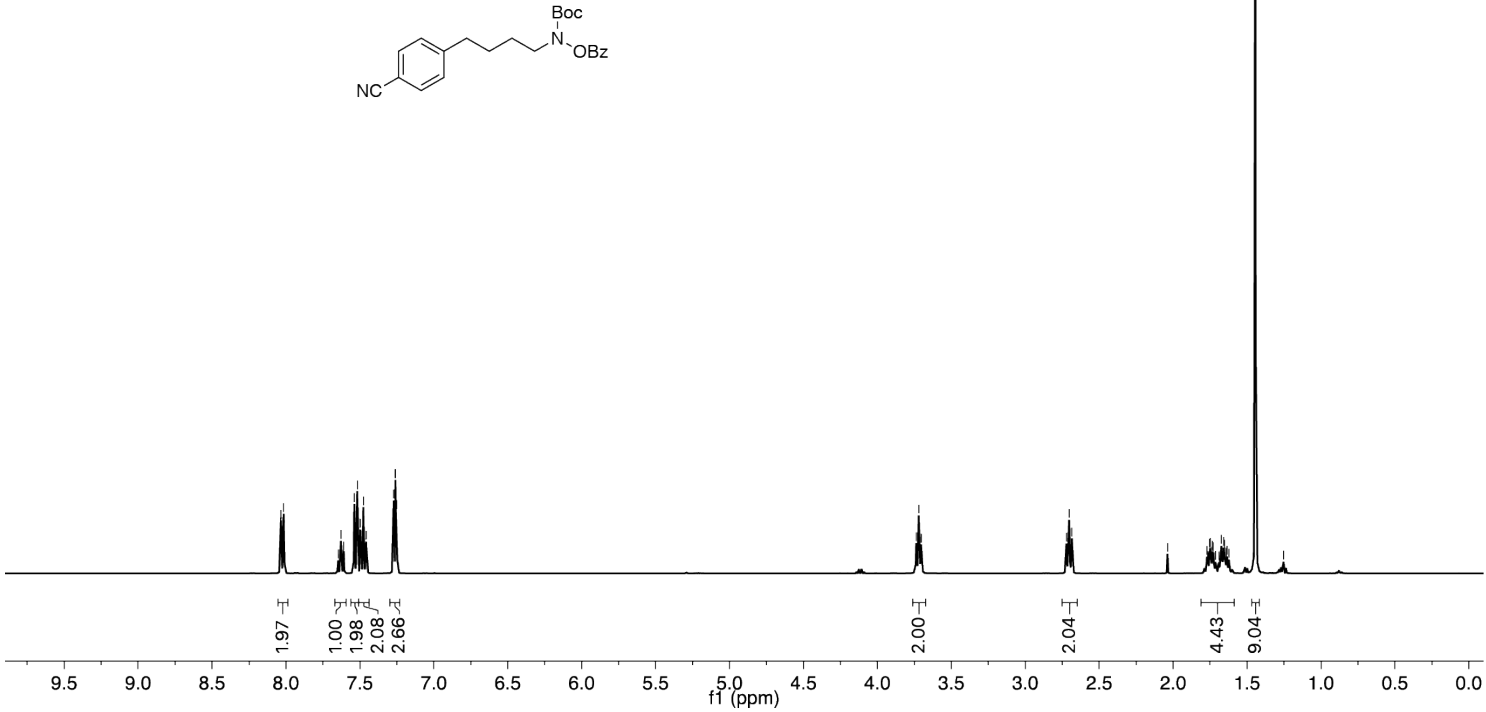

${ }^{13} \mathrm{C}$ NMR $\left(100 \mathrm{MHz}, \mathrm{CDCl}_{3}\right)$
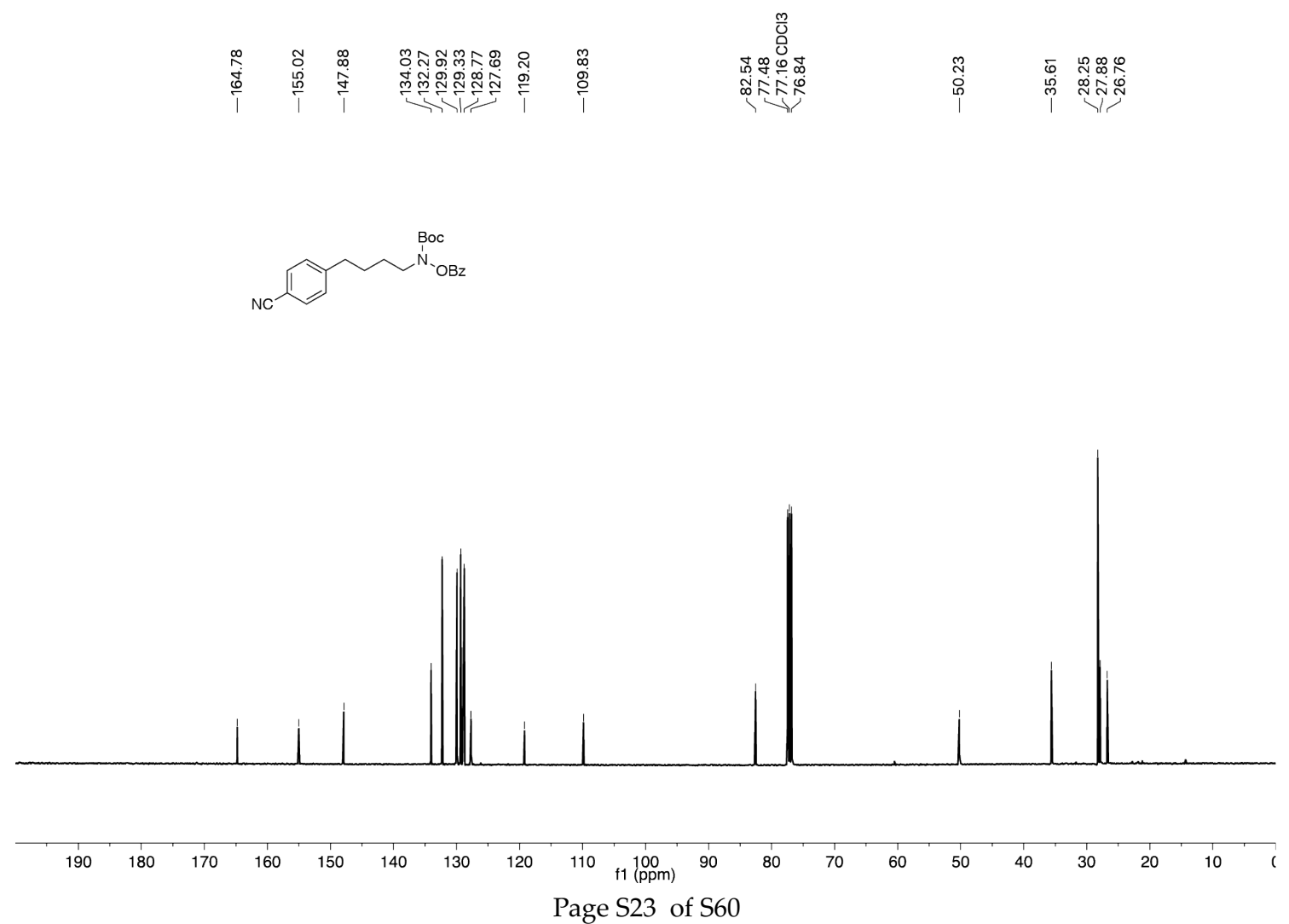

Page S23 of S60 
4-(4-((Benzoyloxy)amino)butyl)benzonitrile (1d):

${ }^{1} \mathbf{H}$ NMR (400 MHz, $\left.\mathrm{CDCl}_{3}\right)$
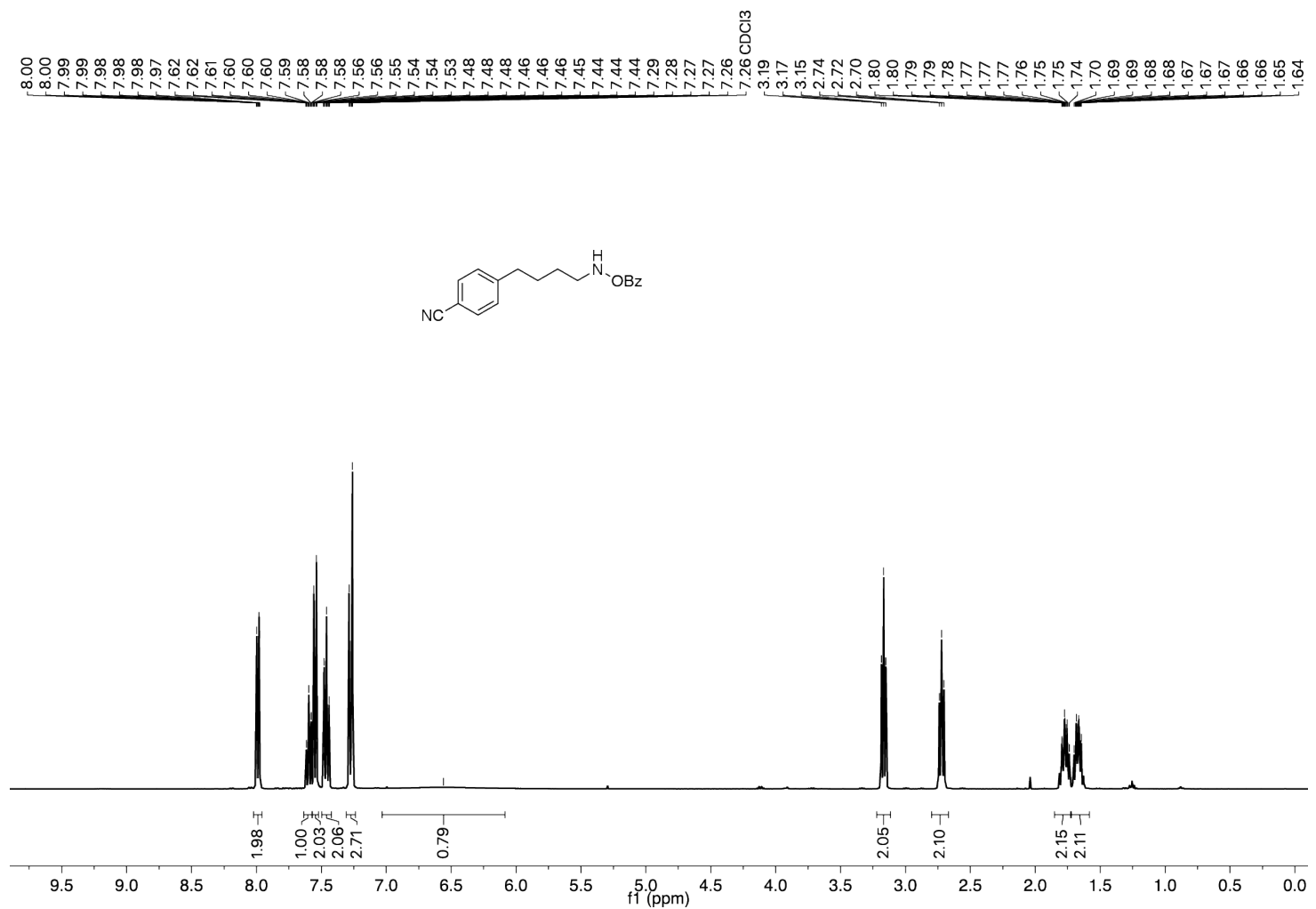

${ }^{13} \mathrm{C}$ NMR (100 MHz, $\left.\mathrm{CDCl}_{3}\right)$

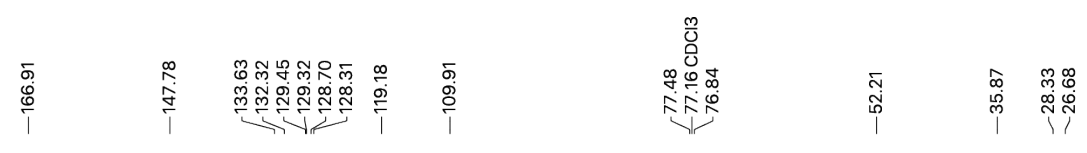
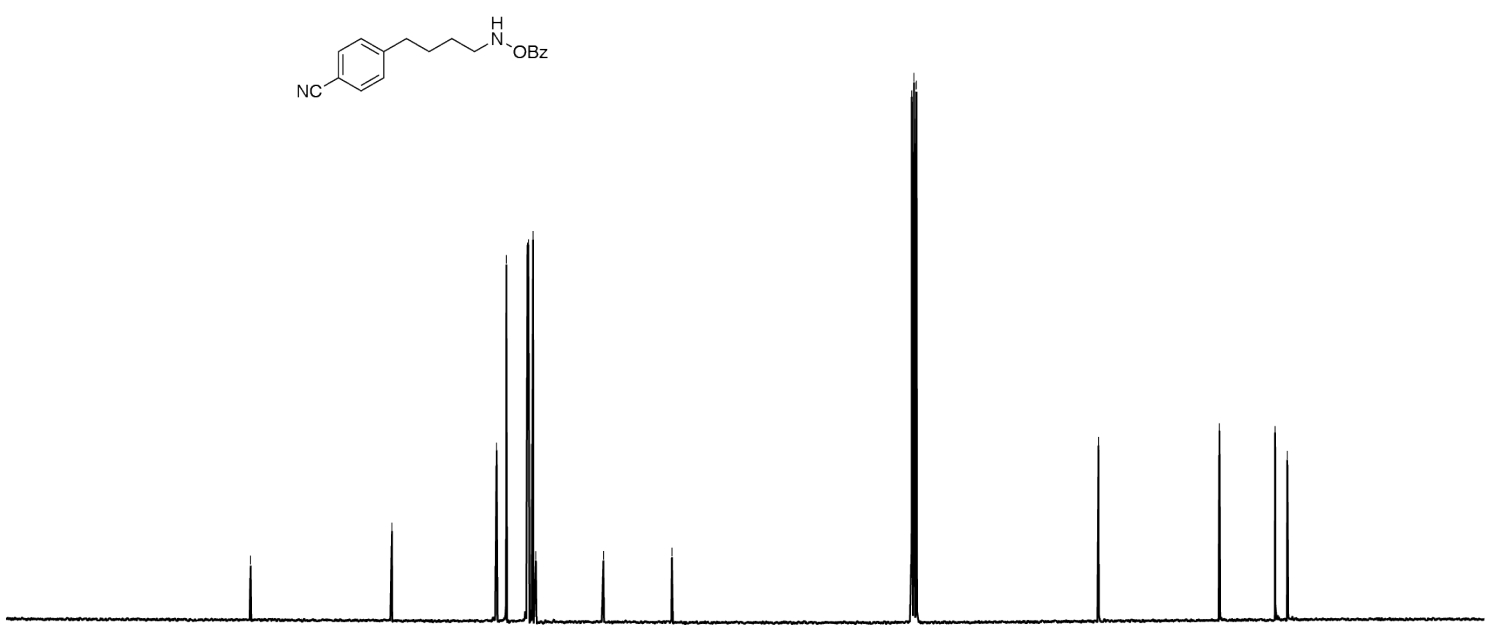

$\begin{array}{llllllll}190 & 180 & 170 & 160 & 150 & 140 & 130 & 120\end{array}$

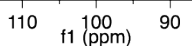

$80 \quad 70$

Page S24 of S60 
Methyl 4-(4-((benzoyloxy)(tert-butoxycarbonyl)amino)butyl)benzoate (Boc-1e):

${ }^{1} \mathbf{H}$ NMR (400 MHz, $\left.\mathrm{CDCl}_{3}\right)$
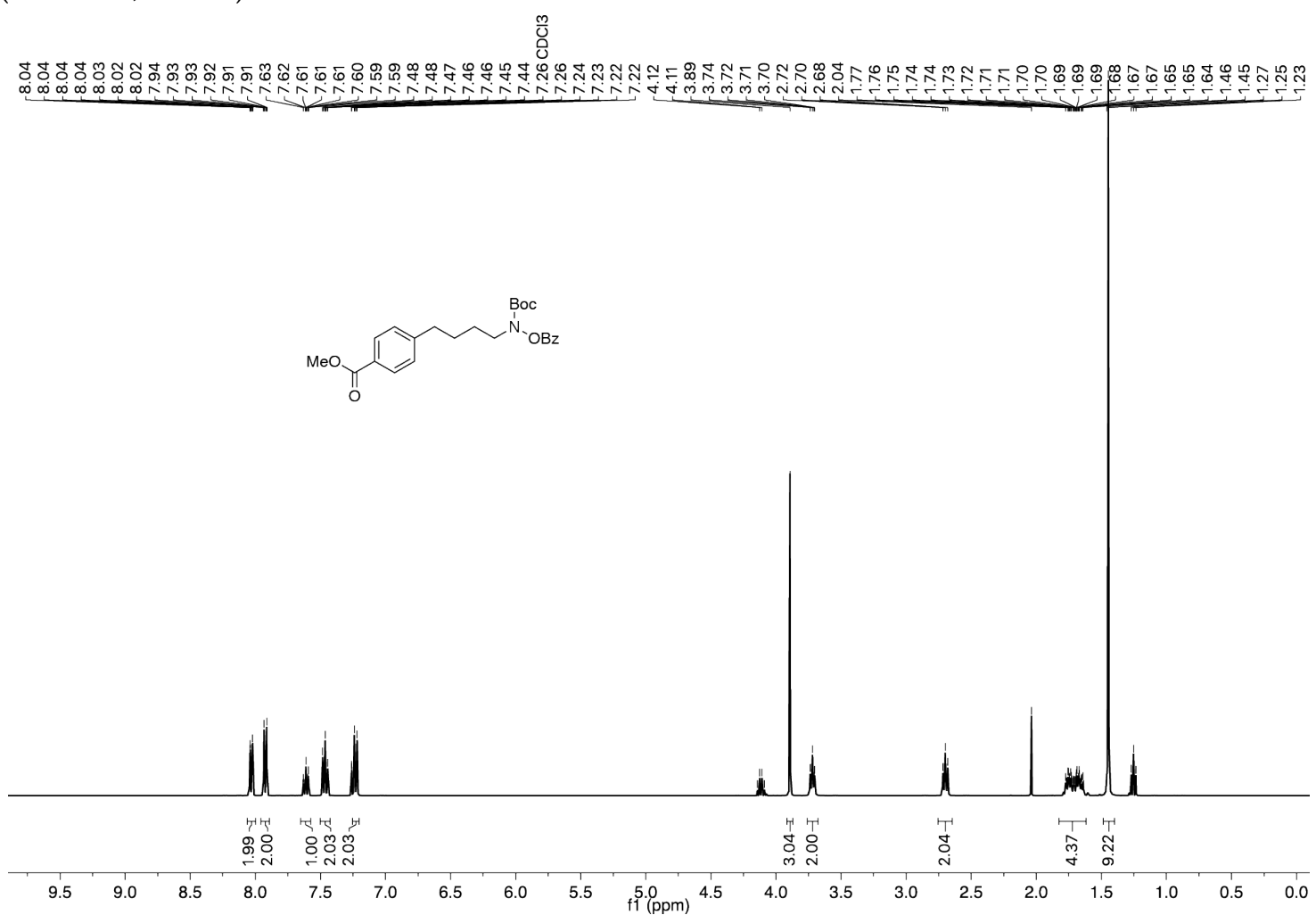

${ }^{13} \mathrm{C}$ NMR (100 MHz, $\left.\mathrm{CDCl}_{3}\right)$
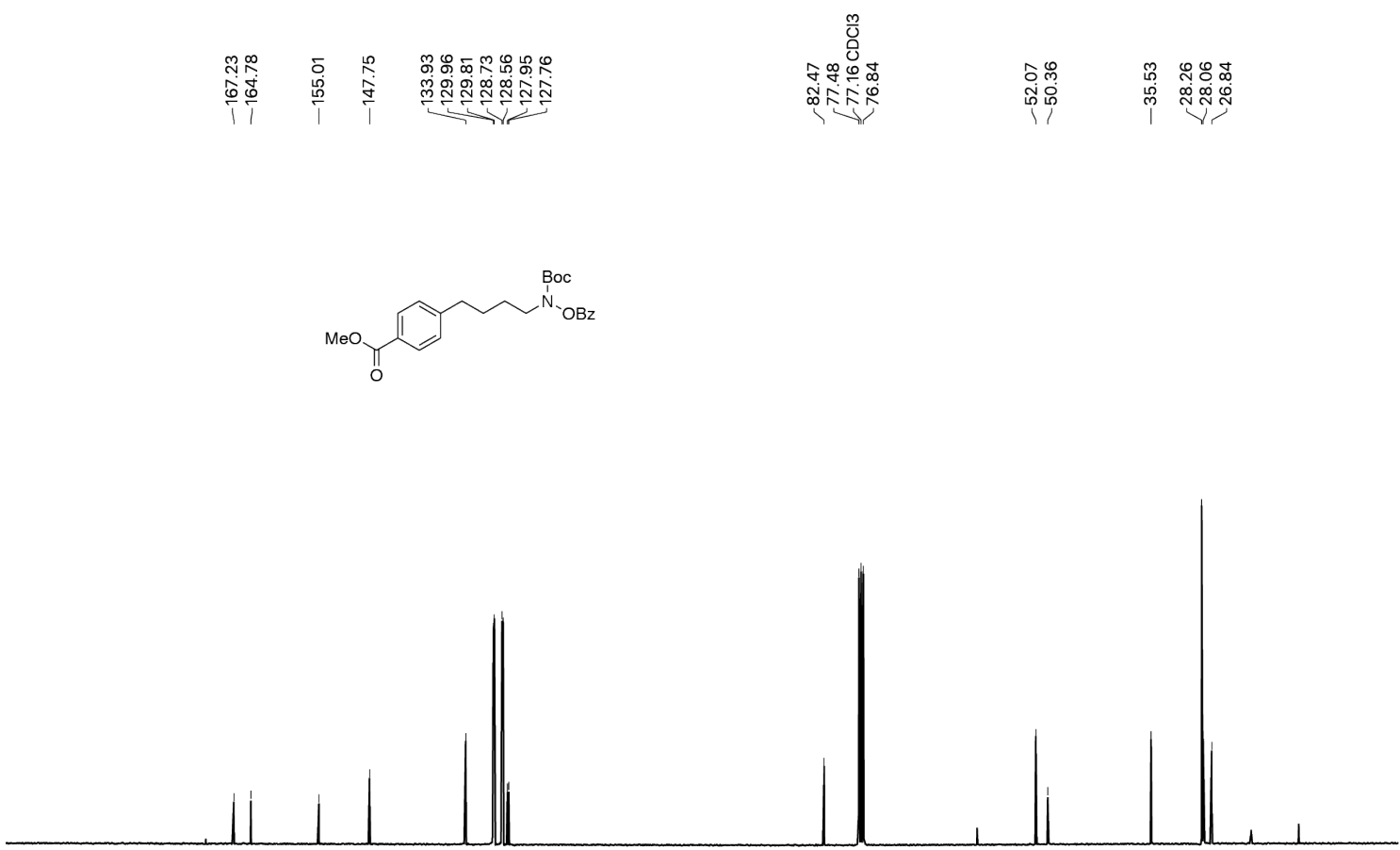

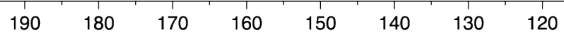

$110+1100+100$

Page S25 of S60 
Methyl 4-(4-((benzoyloxy)amino)butyl)benzoate (1e):

${ }^{1} \mathbf{H}$ NMR (400 MHz, $\left.\mathrm{CDCl}_{3}\right)$
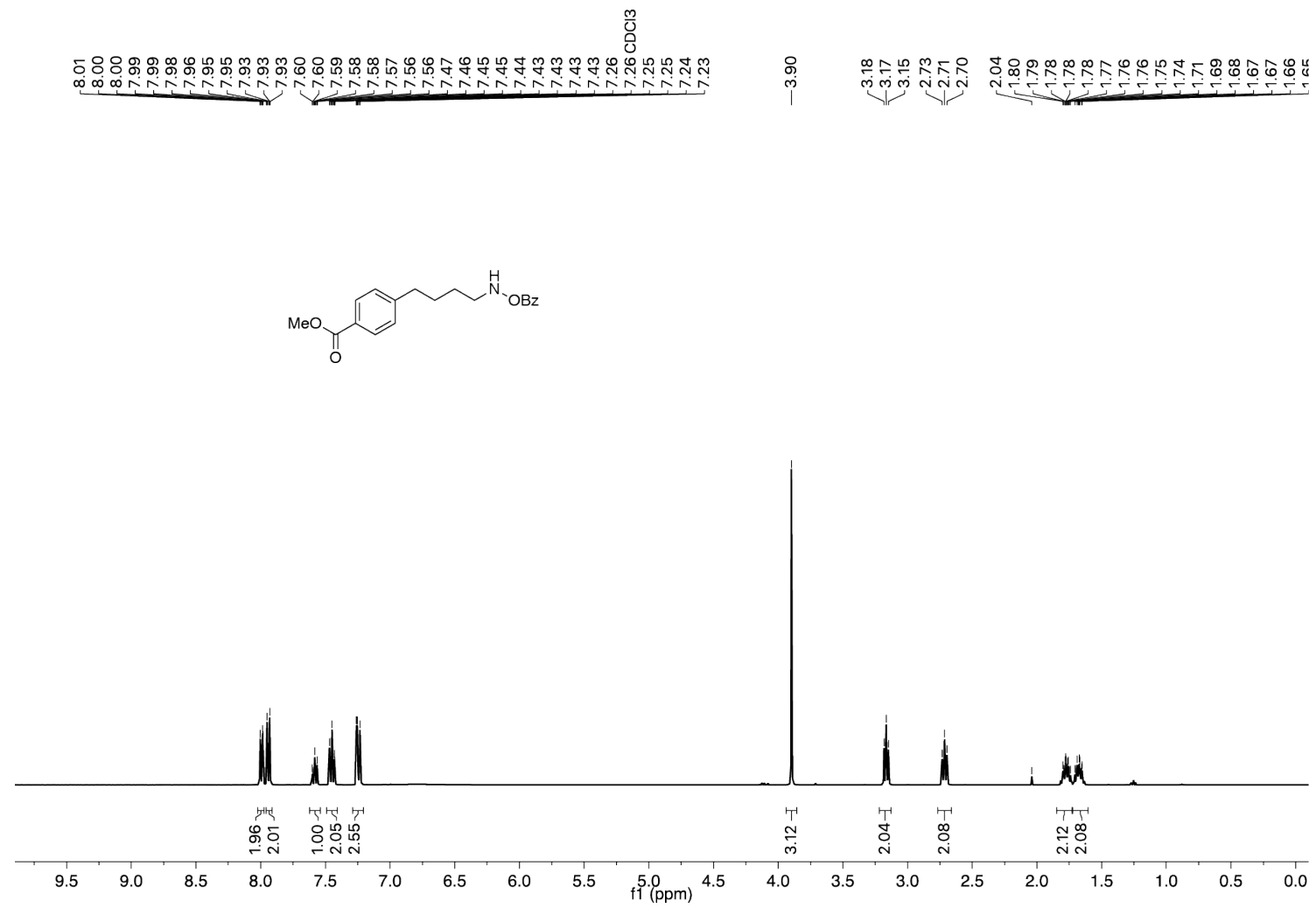

${ }^{13} \mathrm{C}$ NMR (100 MHz, $\left.\mathrm{CDCl}_{3}\right)$

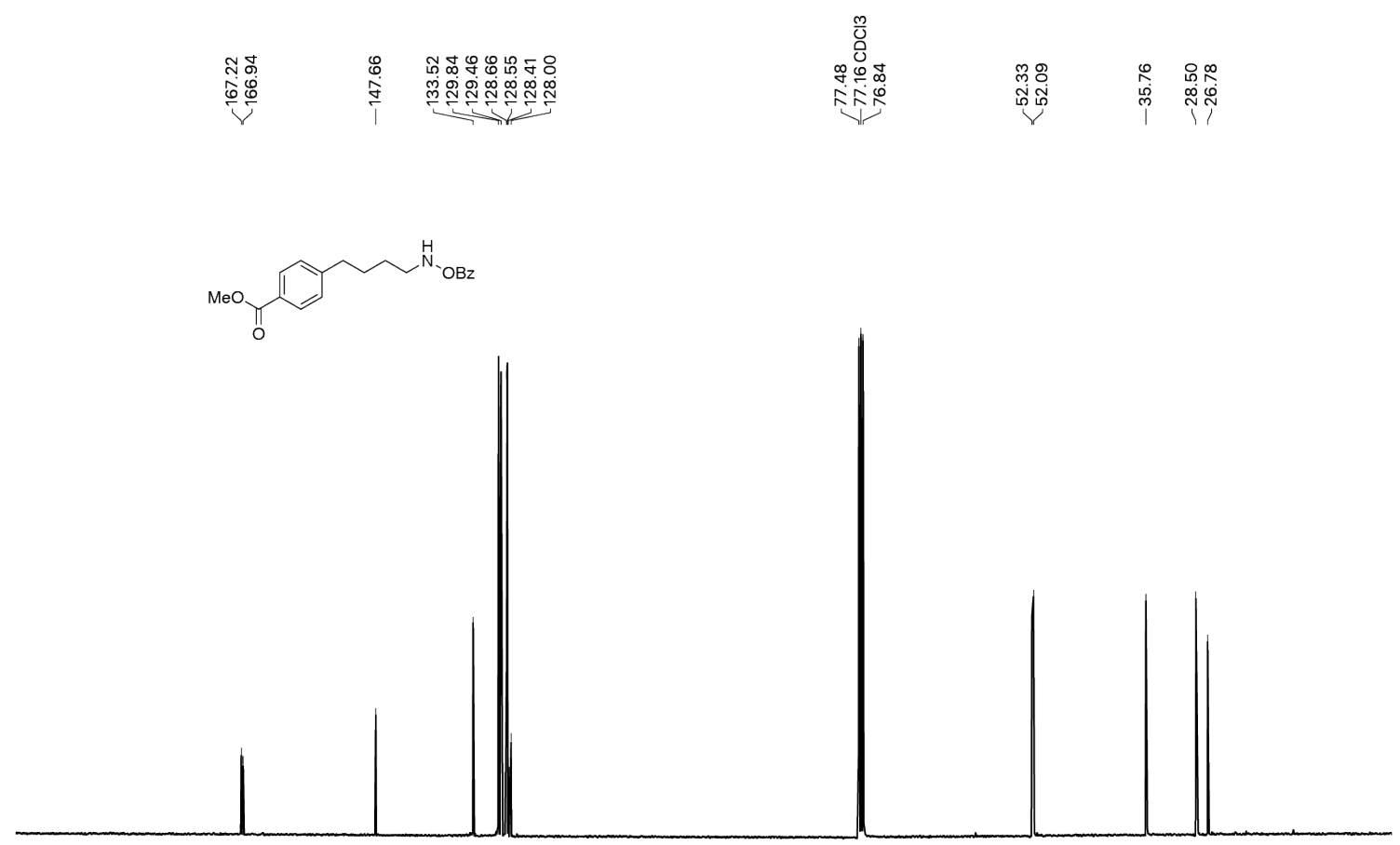

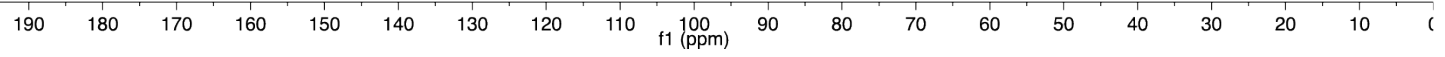

Page S26 of S60 
tert-Butyl (benzoyloxy)(4-(3-methoxyphenyl)butyl)carbamate (Boc-1f):

${ }^{1} \mathbf{H}$ NMR $\left(400 \mathrm{MHz}, \mathrm{CDCl}_{3}\right)$
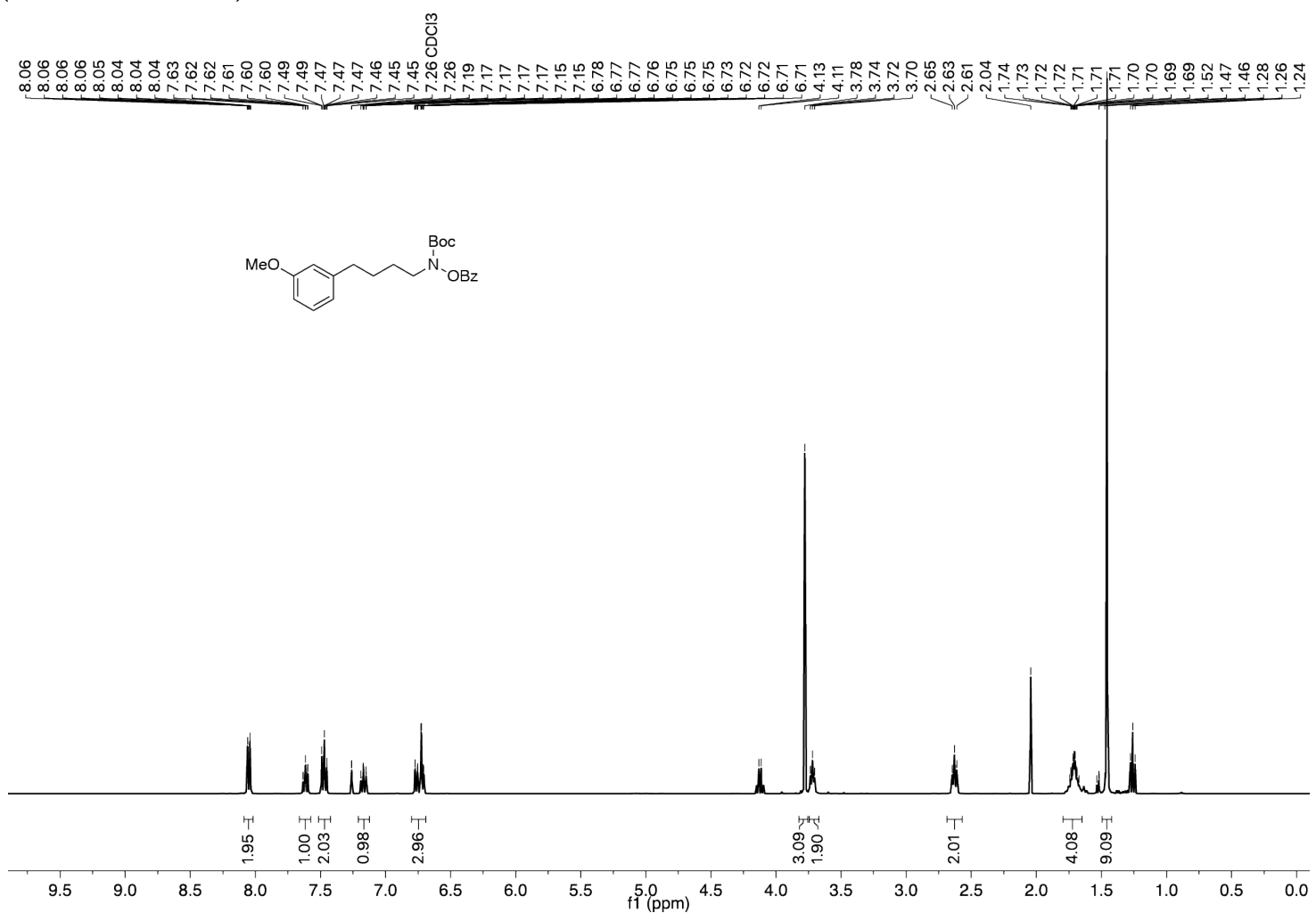

${ }^{13} \mathrm{C}$ NMR (100 MHz, $\left.\mathrm{CDCl}_{3}\right)$

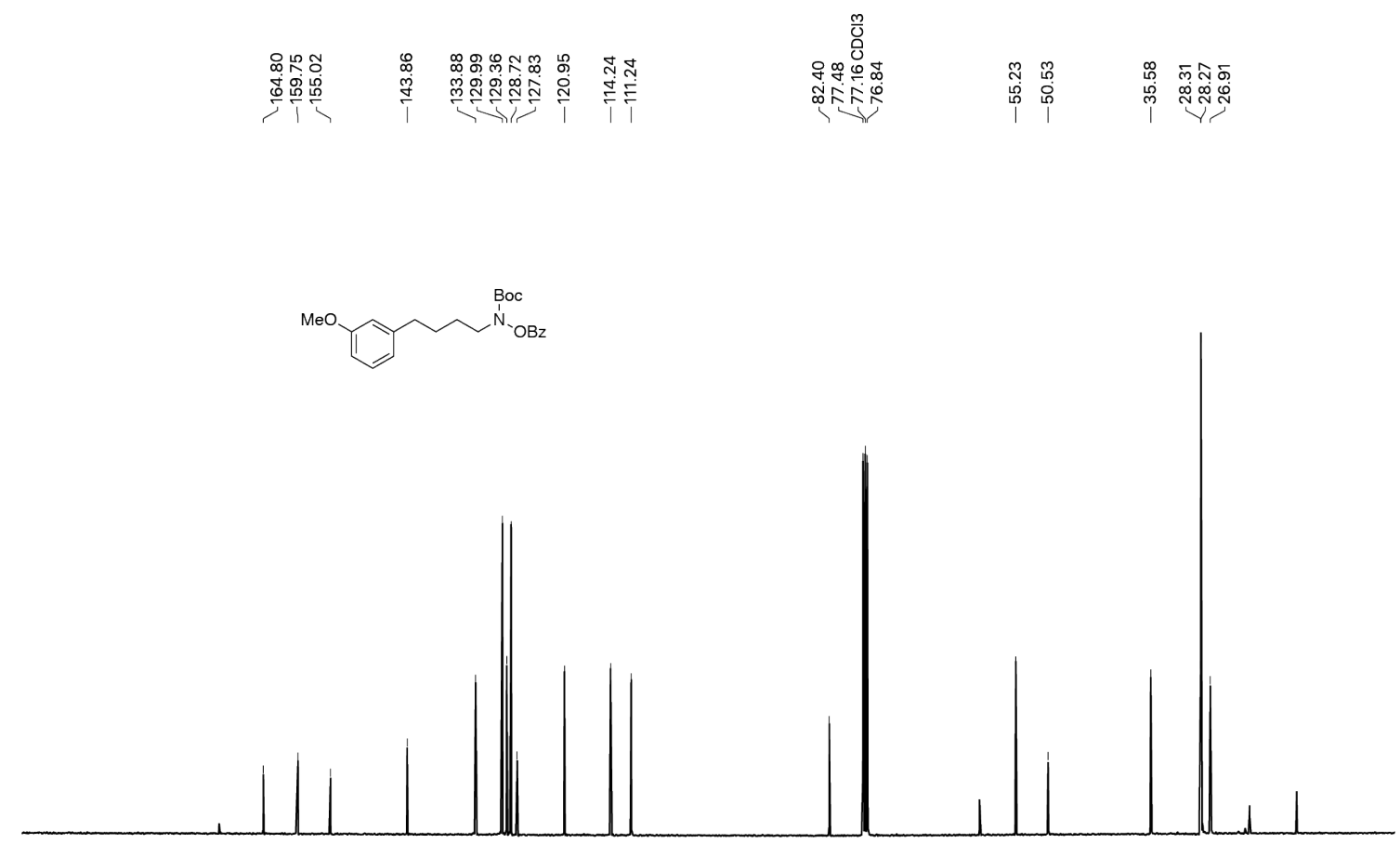


O-Benzoyl-N-(4-(3-methoxyphenyl)butyl)hydroxylamine (1f):

${ }^{1} \mathbf{H}$ NMR (400 MHz, $\left.\mathrm{CDCl}_{3}\right)$
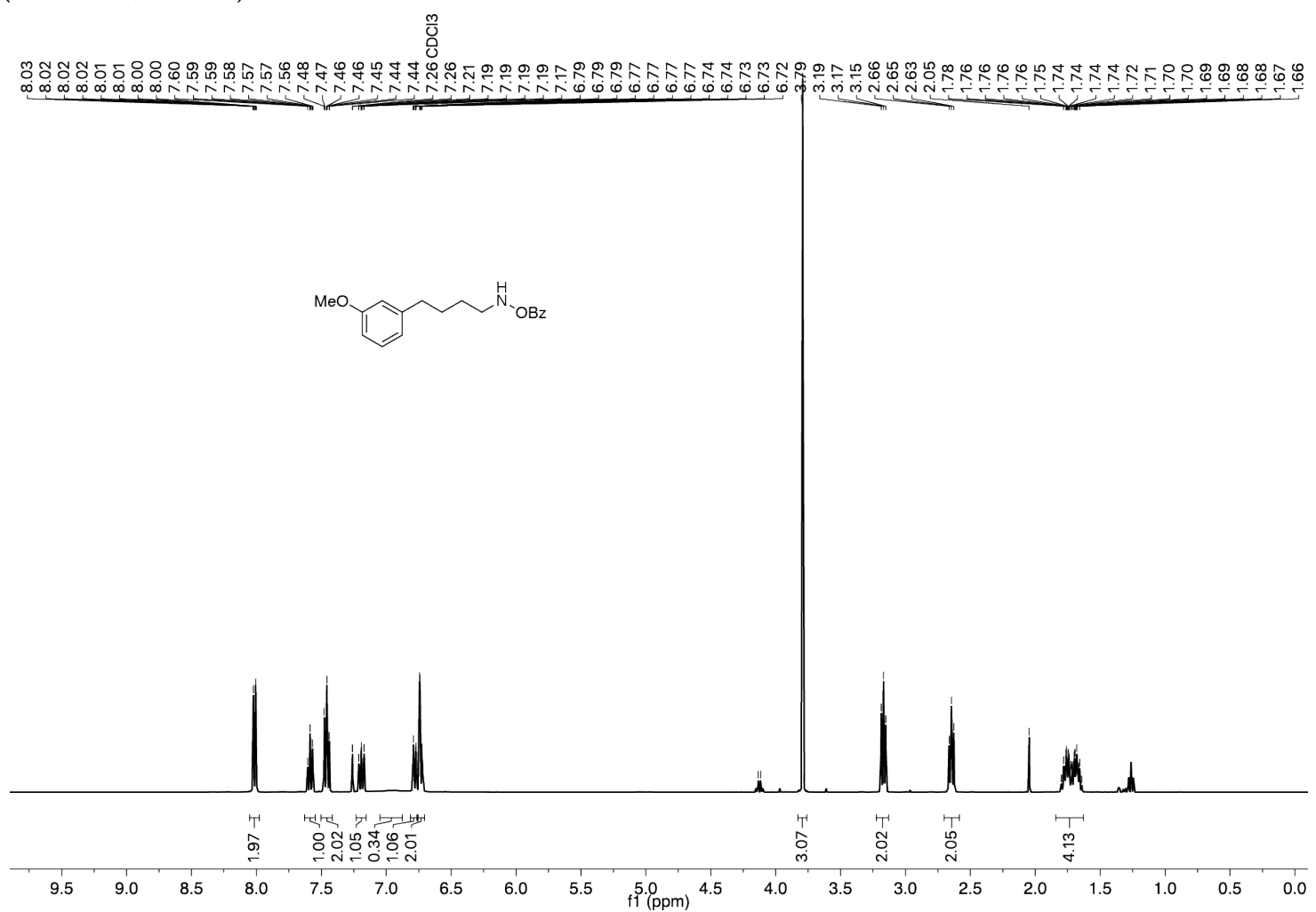

${ }^{13} \mathrm{C}$ NMR (100 MHz, $\left.\mathrm{CDCl}_{3}\right)$
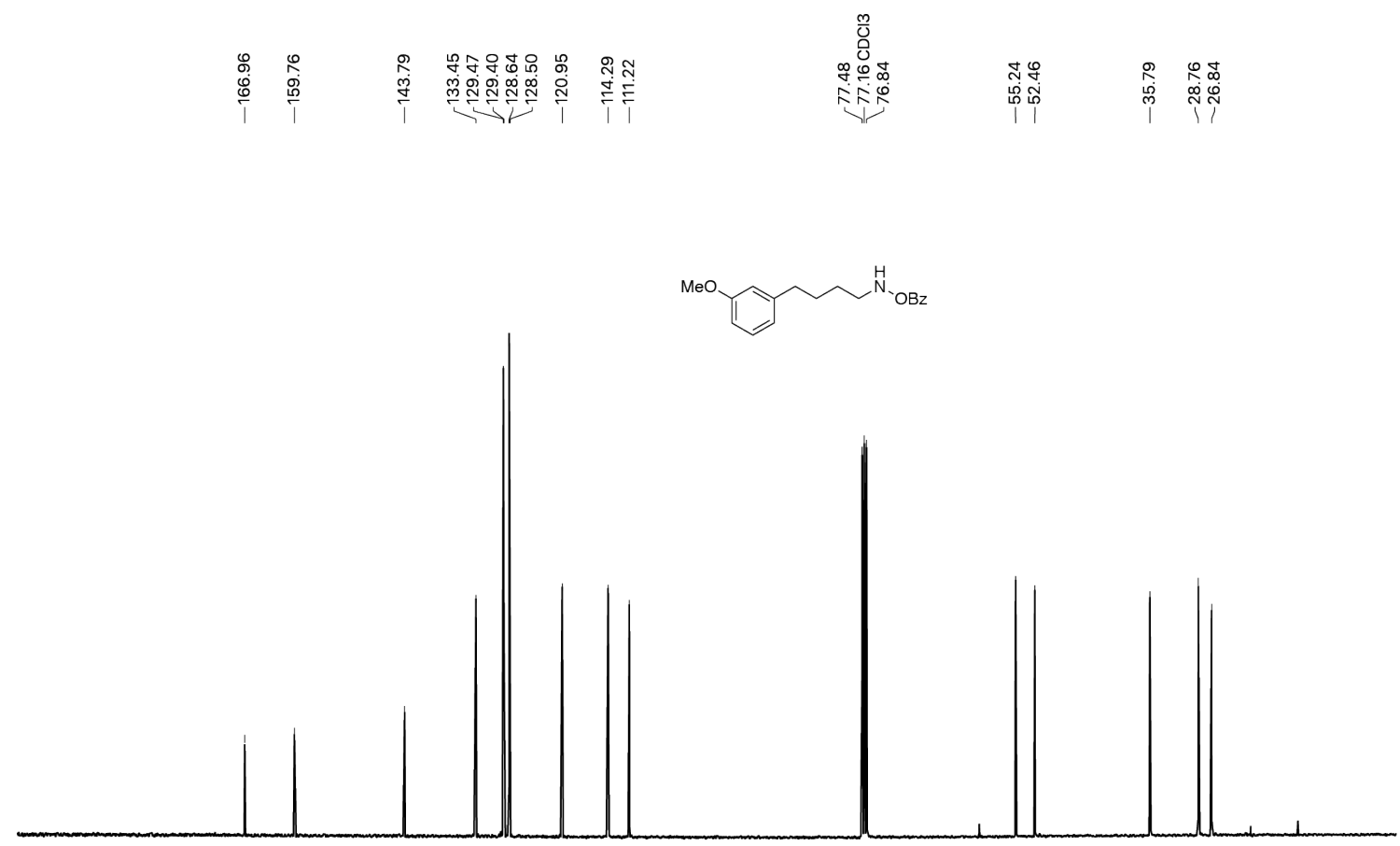

$\begin{array}{llllllll}190 & 180 & 170 & 160 & 150 & 140 & 130 & 120\end{array}$

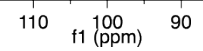

Page S28 of S60 
Methyl 2-(4-((benzoyloxy)(tert-butoxycarbonyl)amino)butyl)benzoate (Boc-1g):

${ }^{1} \mathbf{H}$ NMR $\left(400 \mathrm{MHz}, \mathrm{CDCl}_{3}\right)$
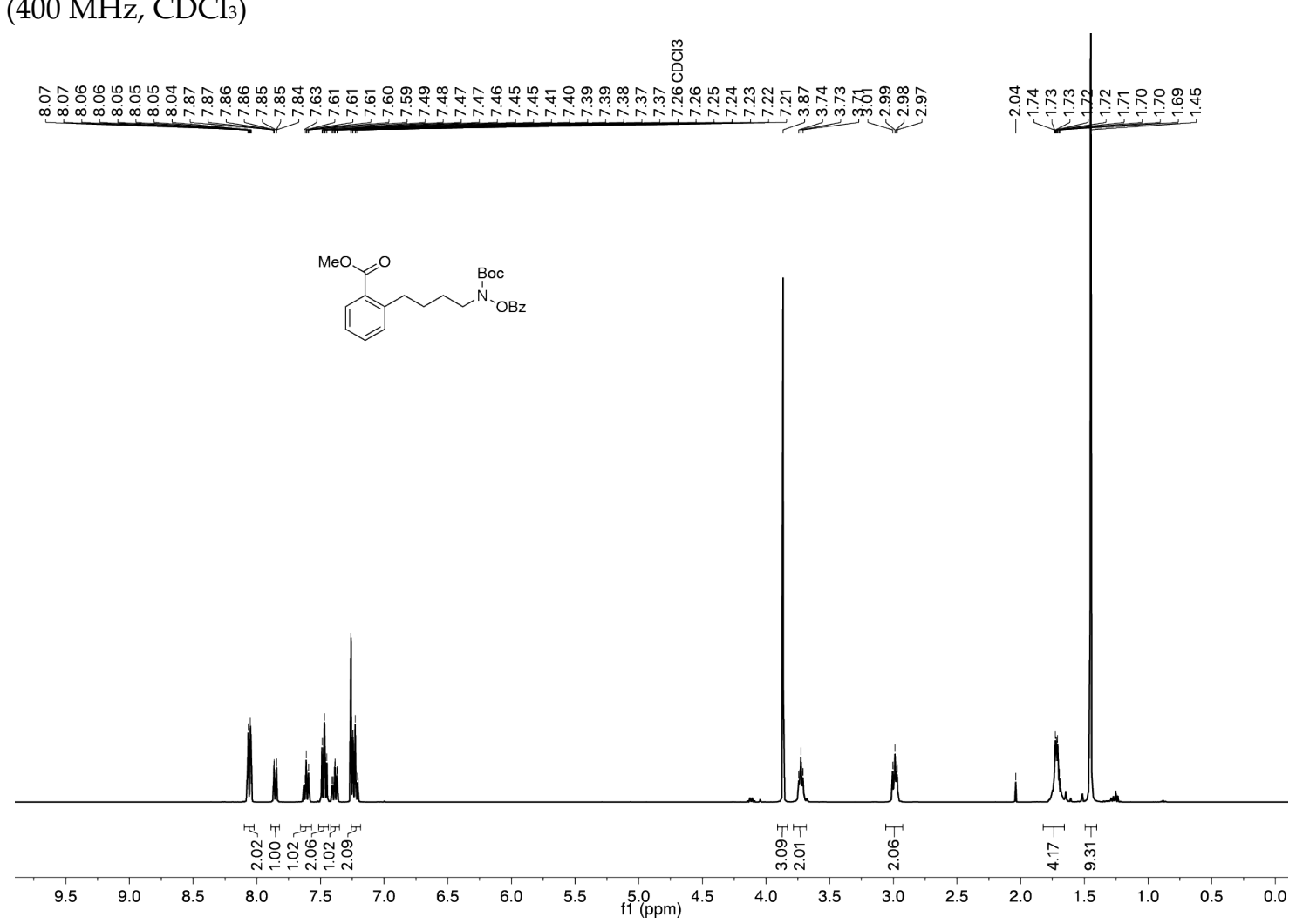

${ }^{13} \mathrm{C}$ NMR (100 MHz, $\left.\mathrm{CDCl}_{3}\right)$
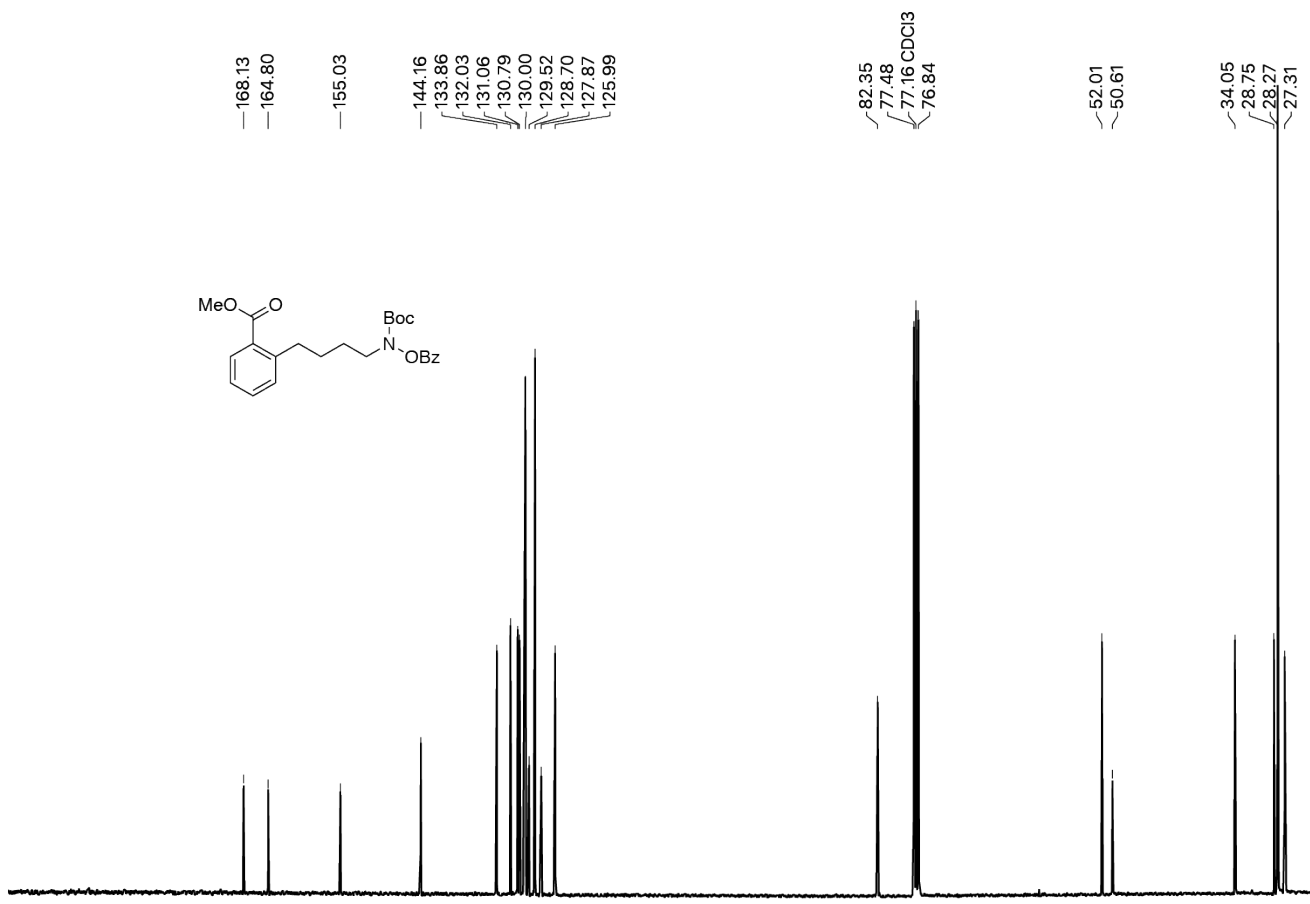

$\begin{array}{llllllll}190 & 180 & 170 & 160 & 150 & 140 & 130 & 120\end{array}$
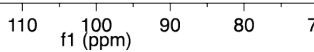

Page S29 of S60 
Methyl 2-(4-((benzoyloxy)amino)butyl)benzoate (1g):

${ }^{1} \mathbf{H}$ NMR (400 MHz, $\left.\mathrm{CDCl}_{3}\right)$

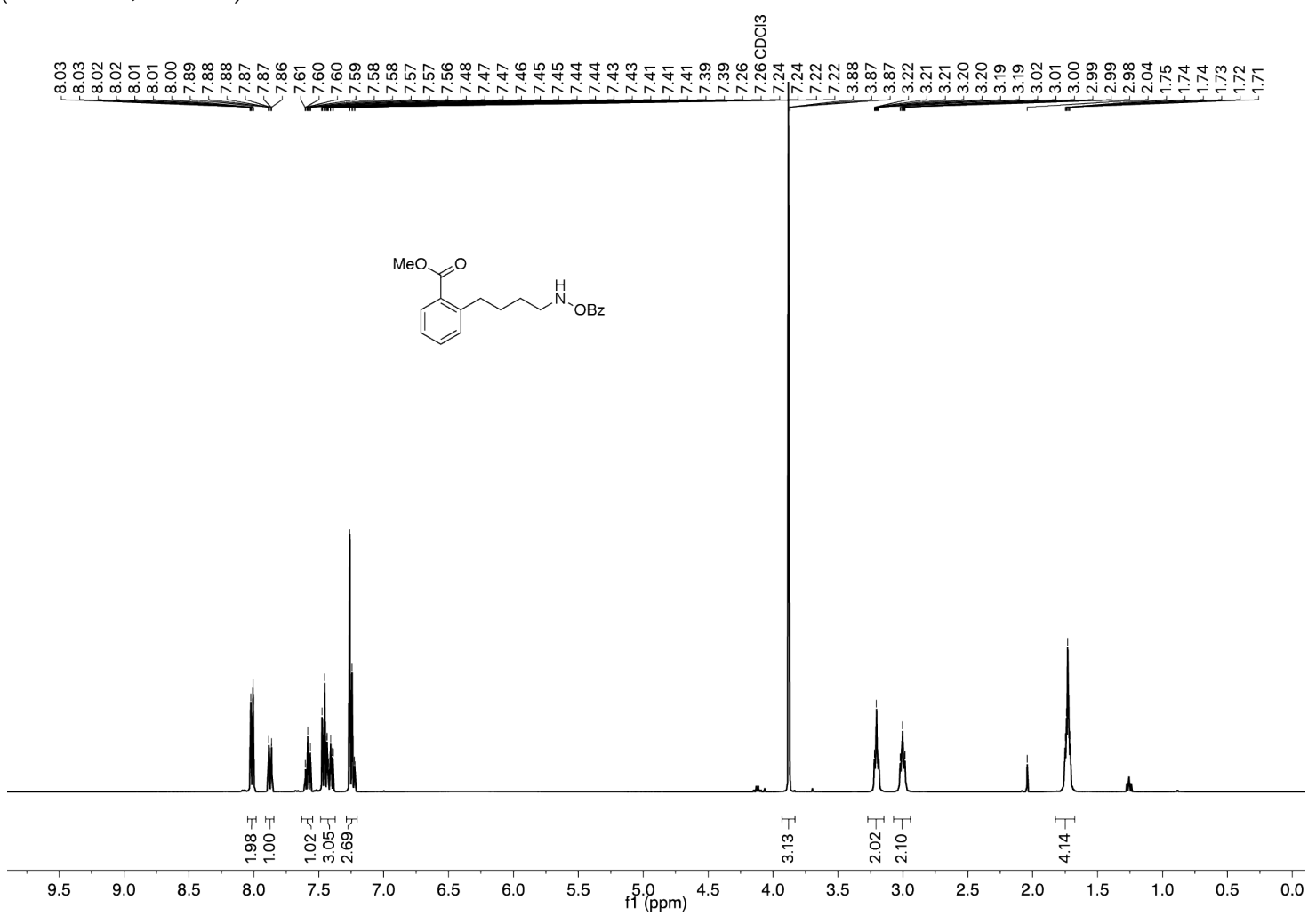

${ }^{13} \mathrm{C}$ NMR (100 MHz, $\left.\mathrm{CDCl}_{3}\right)$

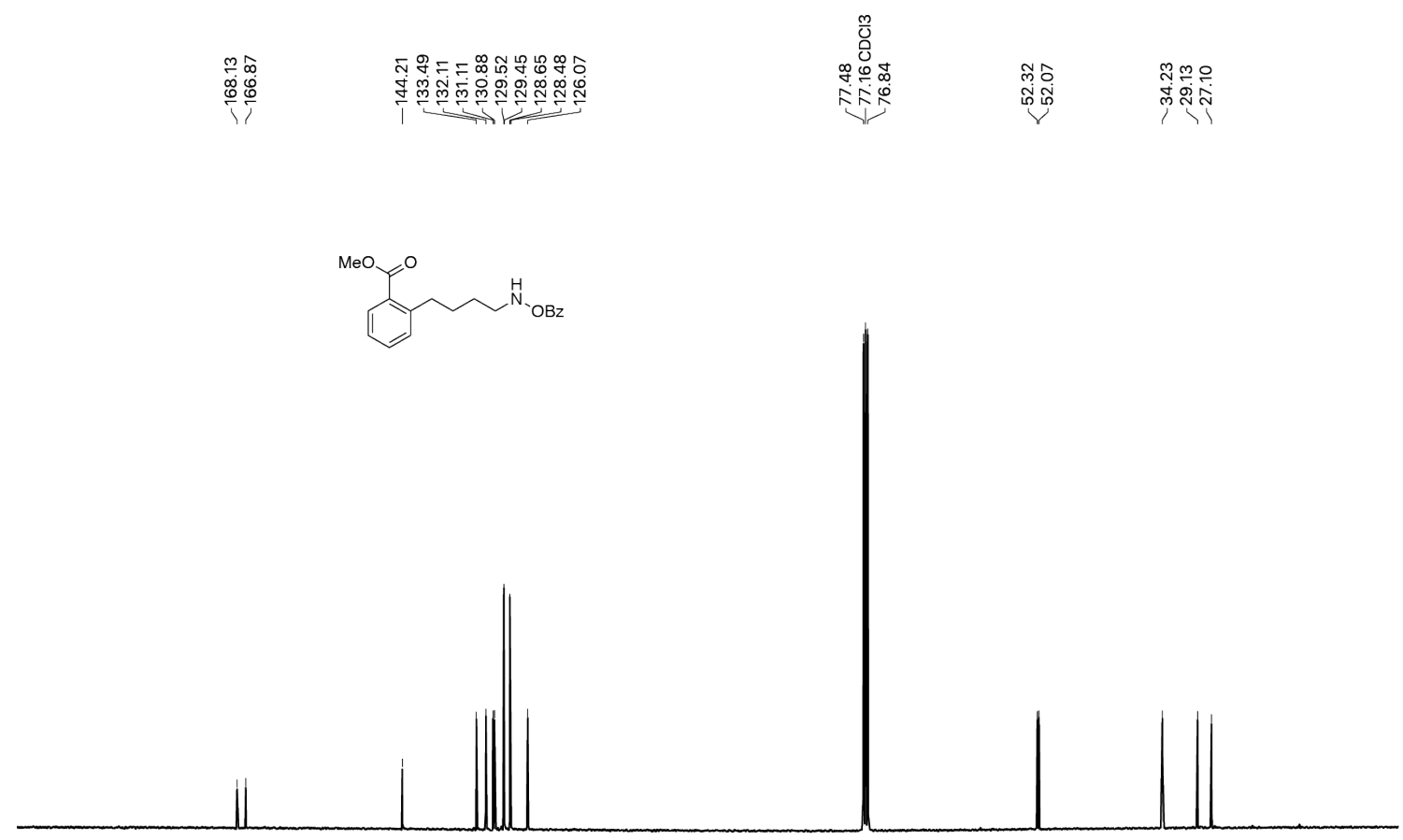

$\begin{array}{llllllll}190 & 180 & 170 & 160 & 150 & 140 & 130 & 120\end{array}$

$110+110000$

$80 \quad 70$

Page S30 of S60 
O-Benzoyl-N-(4-(pyridin-2-yl)butyl)hydroxylamine (1h):

${ }^{1} \mathbf{H}$ NMR $\left(400 \mathrm{MHz}, \mathrm{CDCl}_{3}\right)$
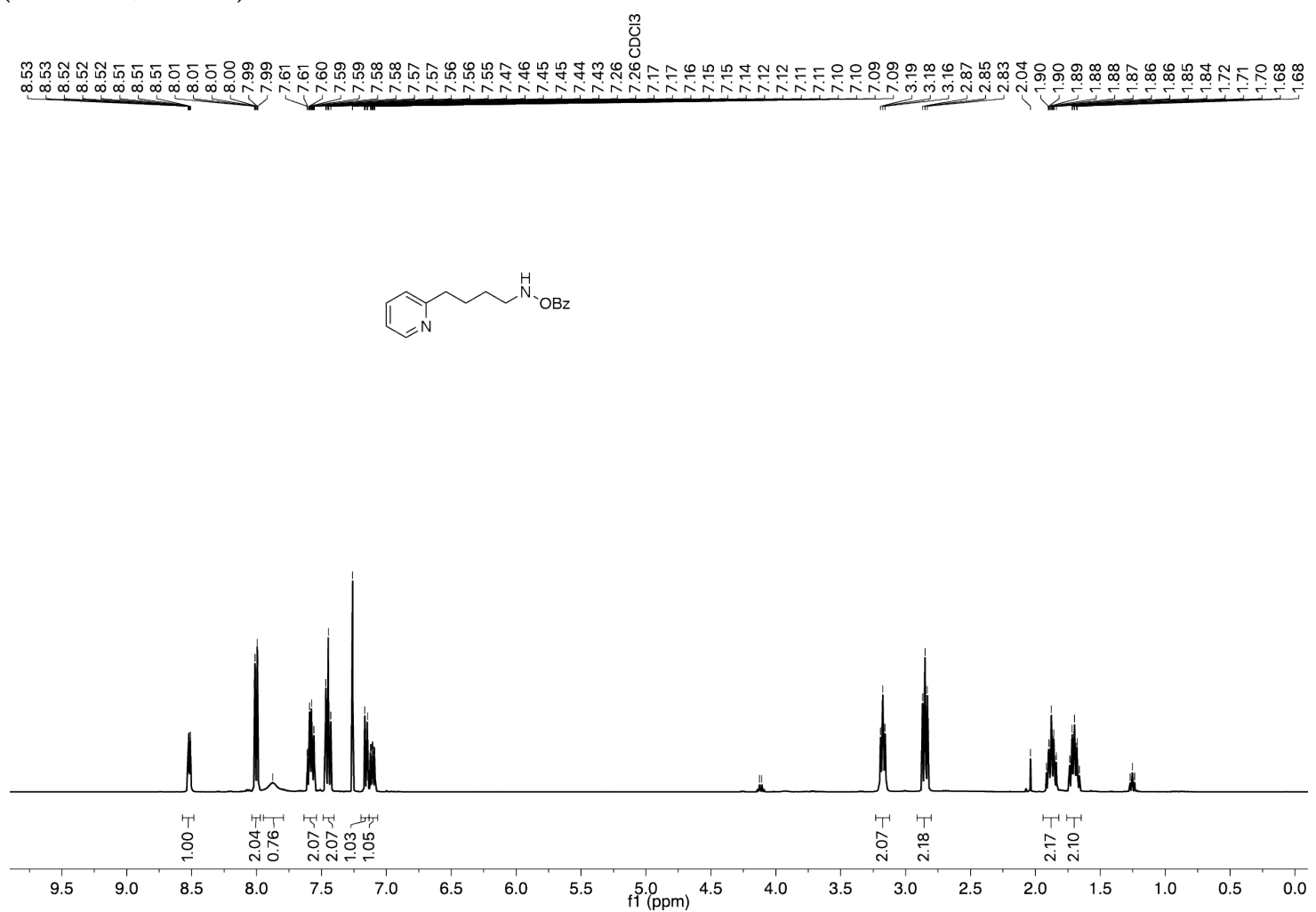

${ }^{13} \mathrm{C}$ NMR (100 MHz, $\left.\mathrm{CDCl}_{3}\right)$

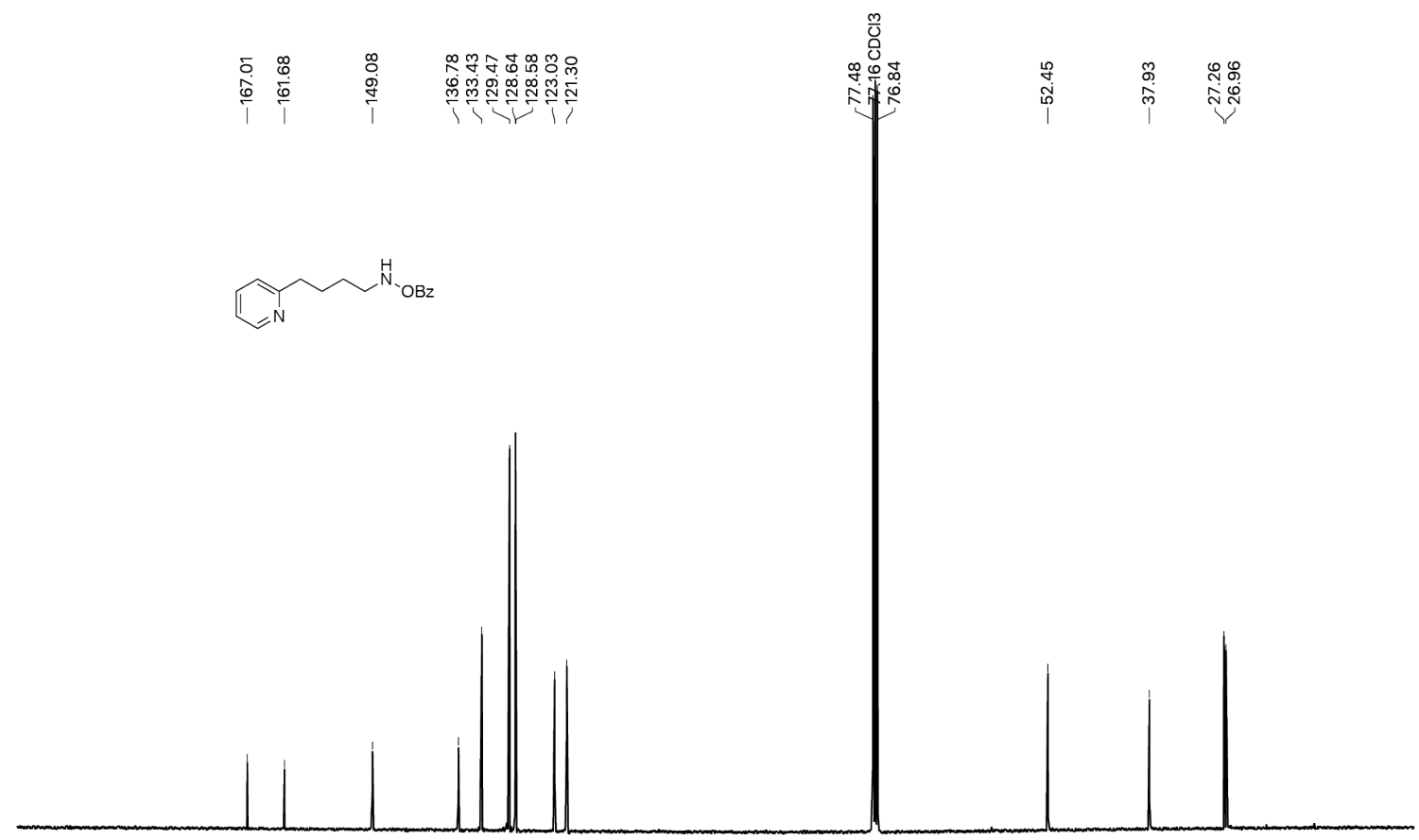

$\begin{array}{llllllll}190 & 180 & 170 & 160 & 150 & 140 & 130 & 120\end{array}$

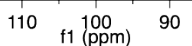

$80 \quad 70$

Page S31 of S60 
O-Benzoyl-N-(5-methylhexan-2-yl)hydroxylamine (1i):

${ }^{1} \mathbf{H}$ NMR $\left(400 \mathrm{MHz}, \mathrm{CDCl}_{3}\right)$
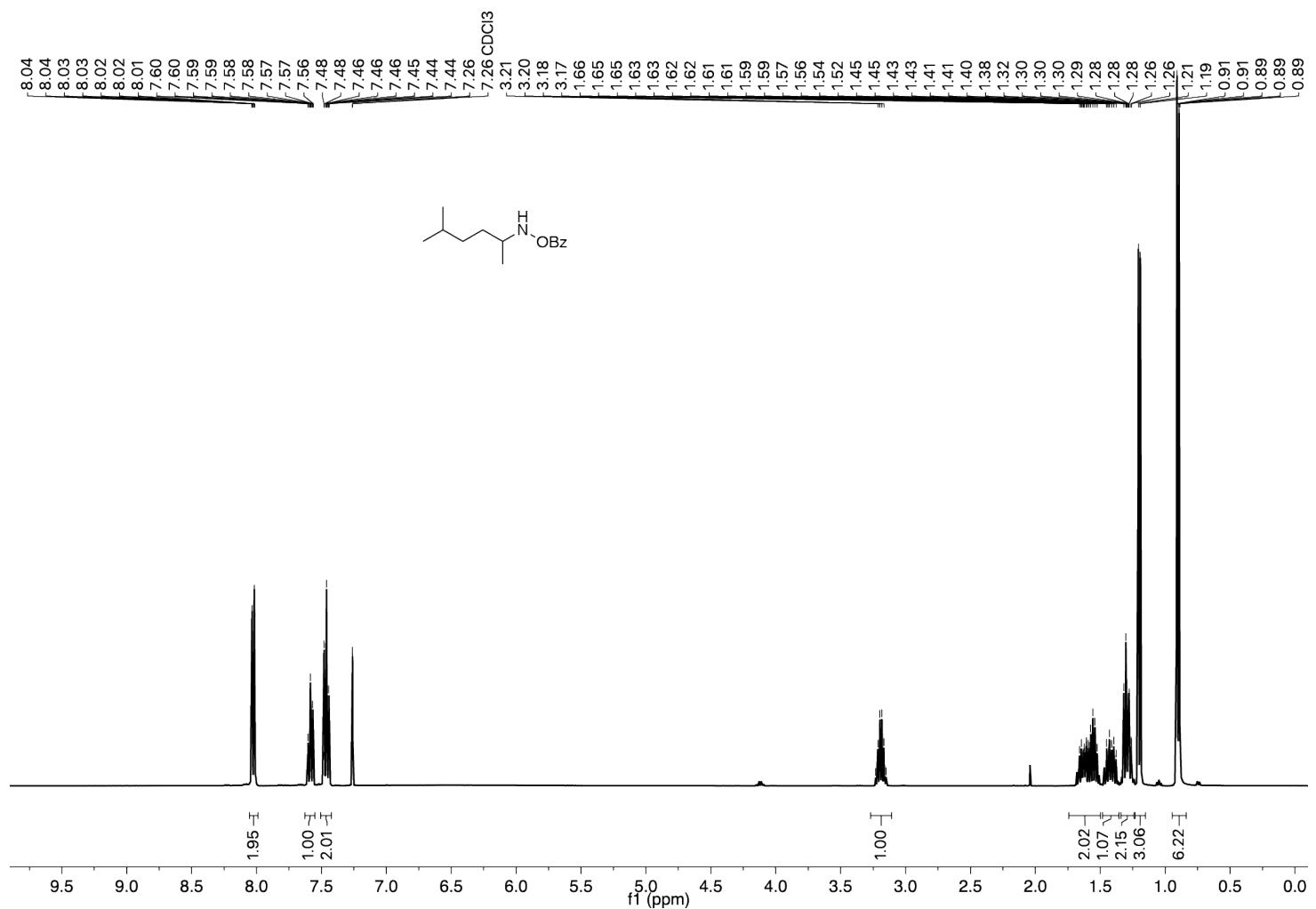

${ }^{13} \mathrm{C}$ NMR (100 MHz, $\left.\mathrm{CDCl}_{3}\right)$

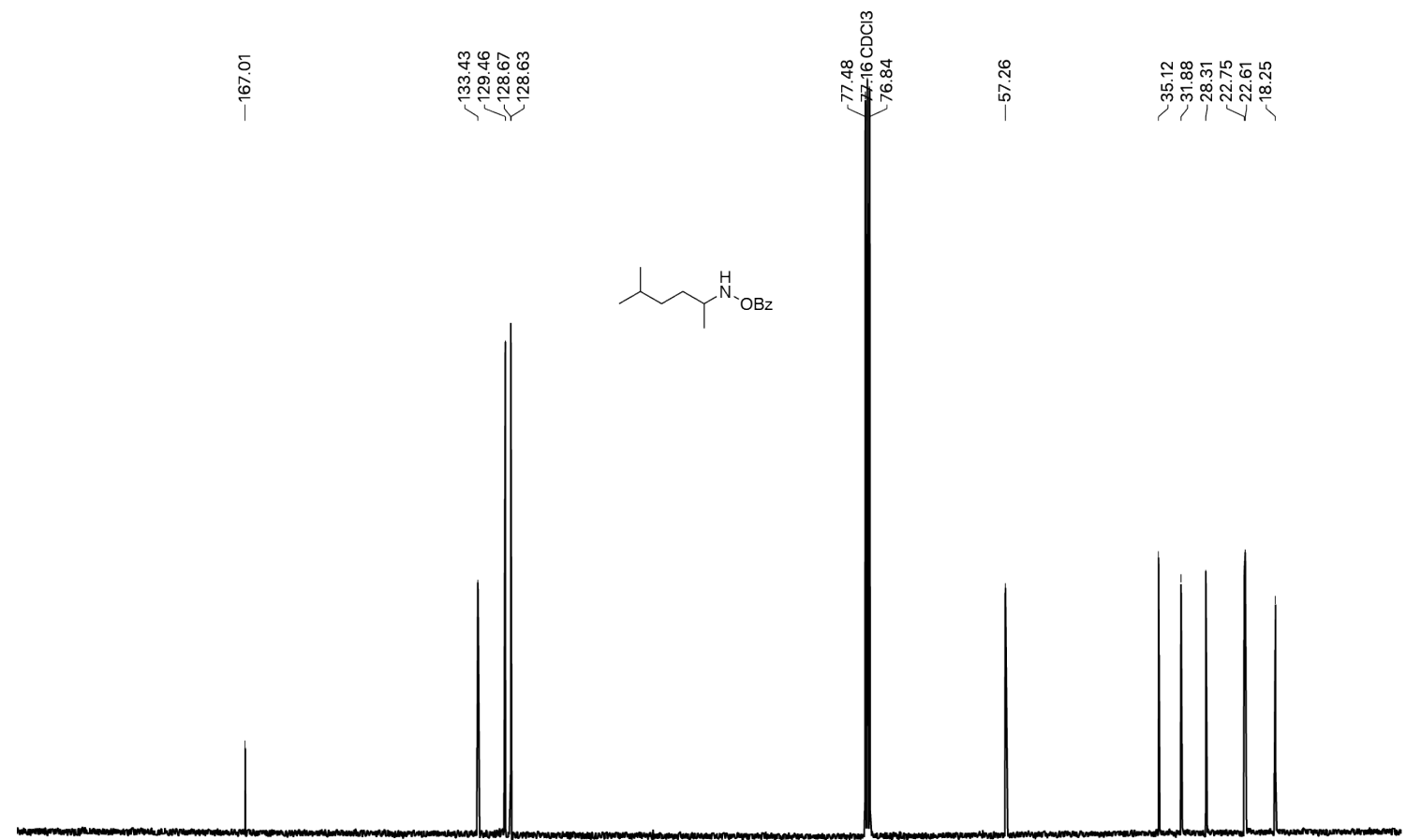

$\begin{array}{llllllll}190 & 180 & 170 & 160 & 150 & 140 & 130 & 120\end{array}$

$110+11000$

Page S32 of S60 


\section{$O$-Benzoyl- $N$-heptylhydroxylamine (1j):}

${ }^{1} \mathbf{H}$ NMR (400 MHz, $\left.\mathrm{CDCl}_{3}\right)$
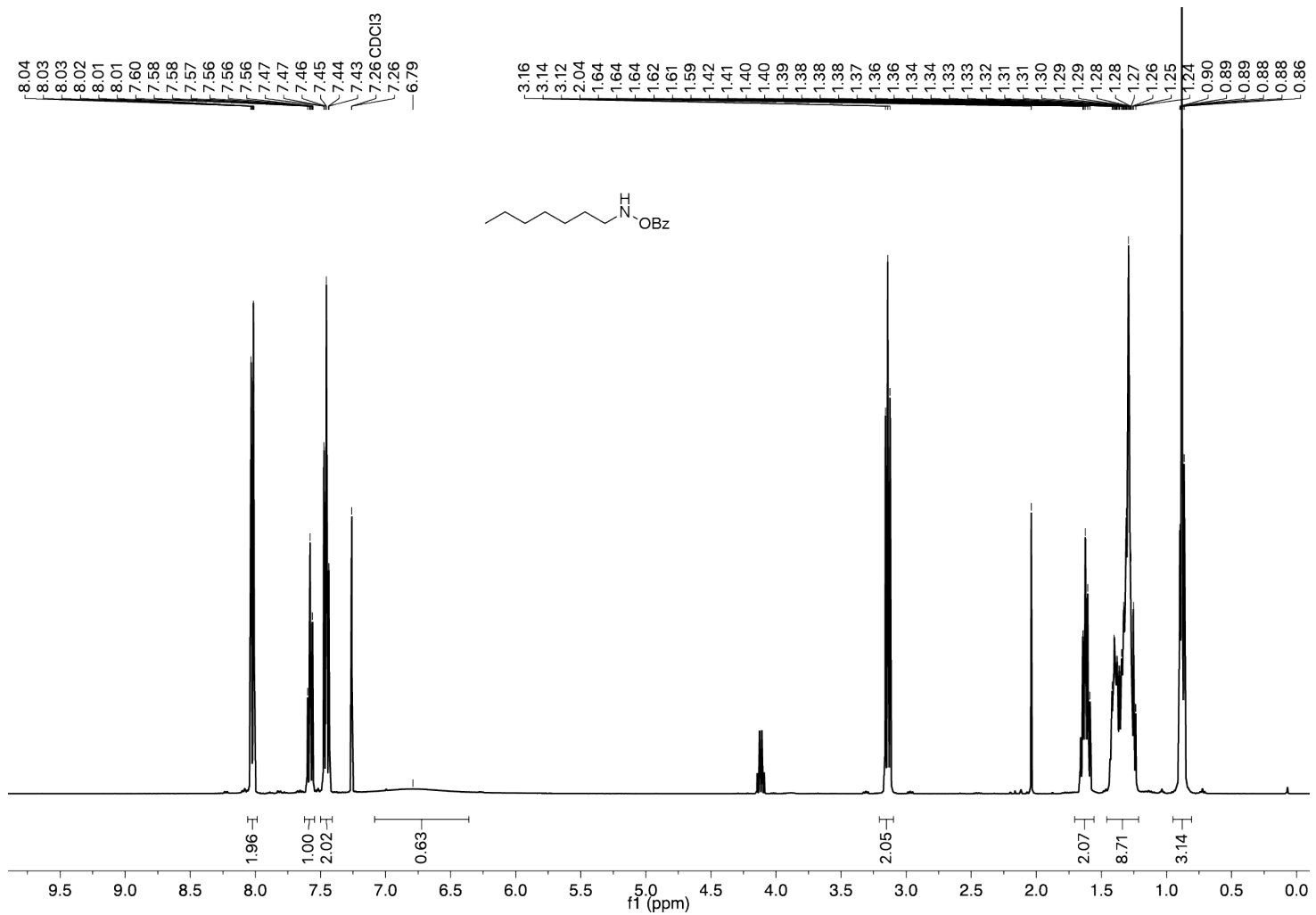

${ }^{13} \mathrm{C}$ NMR (100 MHz, $\left.\mathrm{CDCl}_{3}\right)$

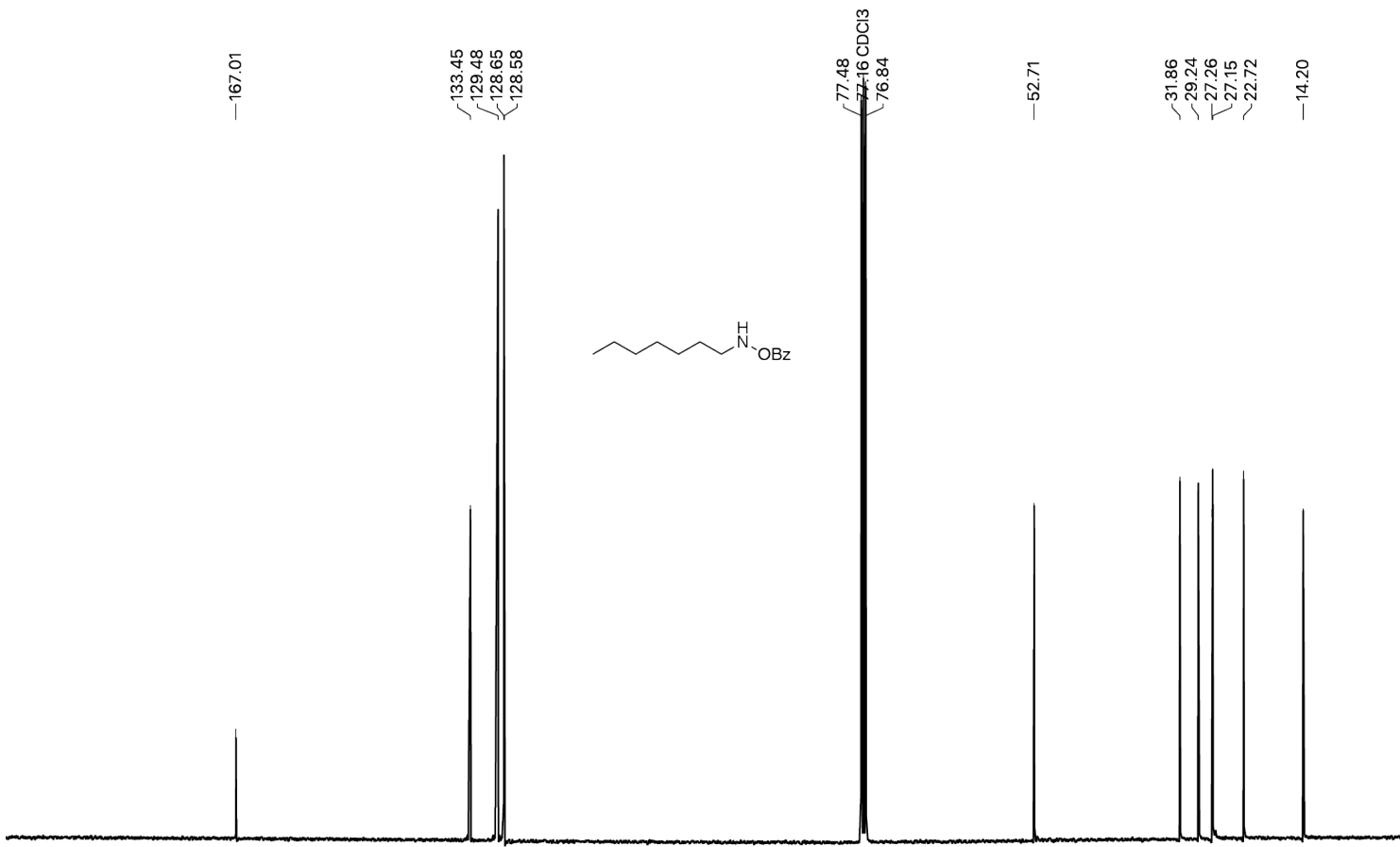

$\begin{array}{llllllll}190 & 180 & 170 & 160 & 150 & 140 & 130 & 120\end{array}$

$110+1 \begin{array}{cc}100 \\ \text { (ppm) }\end{array}$

Page S33 of S60 
tert-Butyl (benzoyloxy)(5-phenylpentyl)carbamate (Boc-1k):

${ }^{1} \mathbf{H}$ NMR $\left(400 \mathrm{MHz}, \mathrm{CDCl}_{3}\right)$
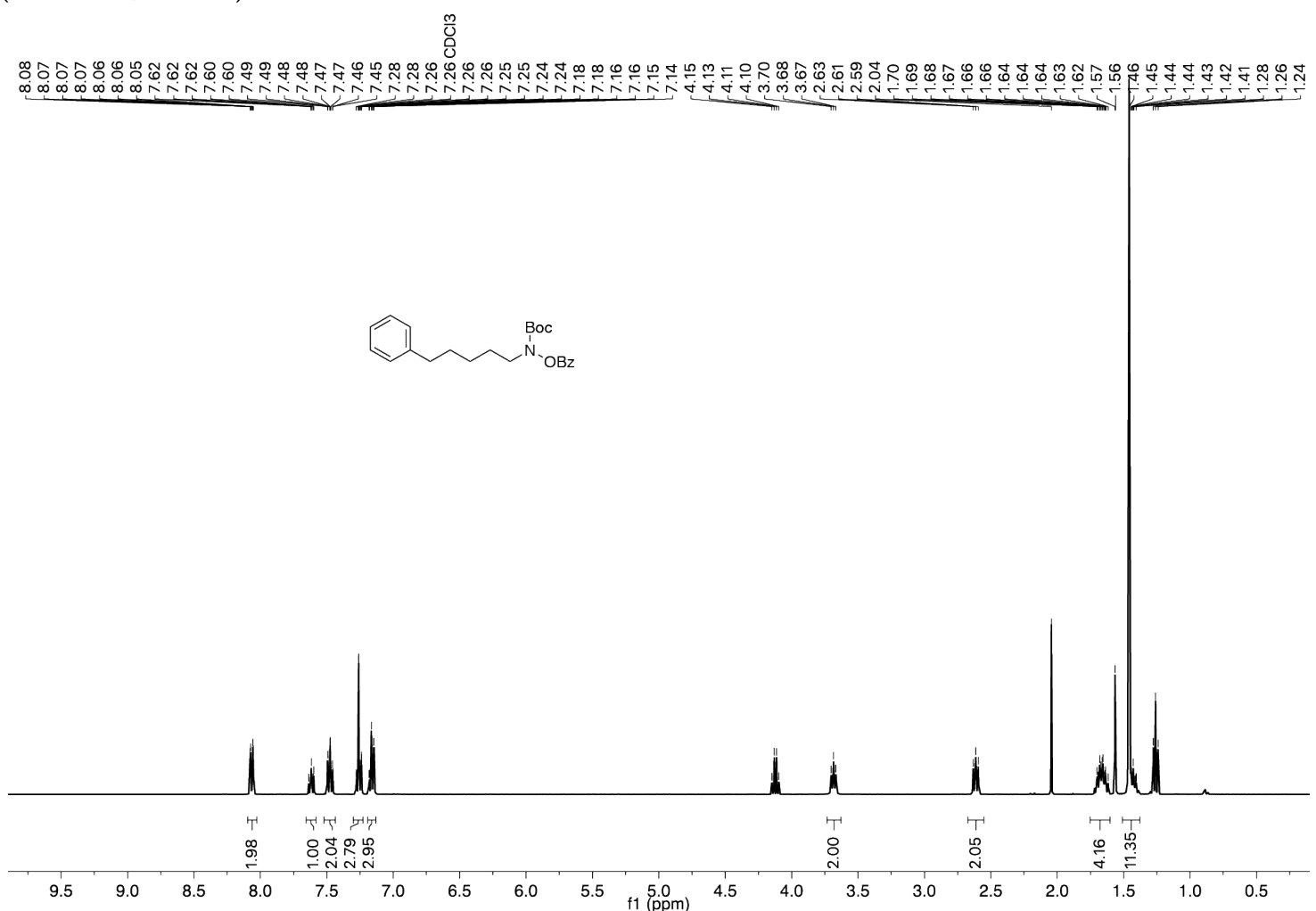

${ }^{13} \mathrm{C}$ NMR $\left(100 \mathrm{MHz}, \mathrm{CDCl}_{3}\right)$

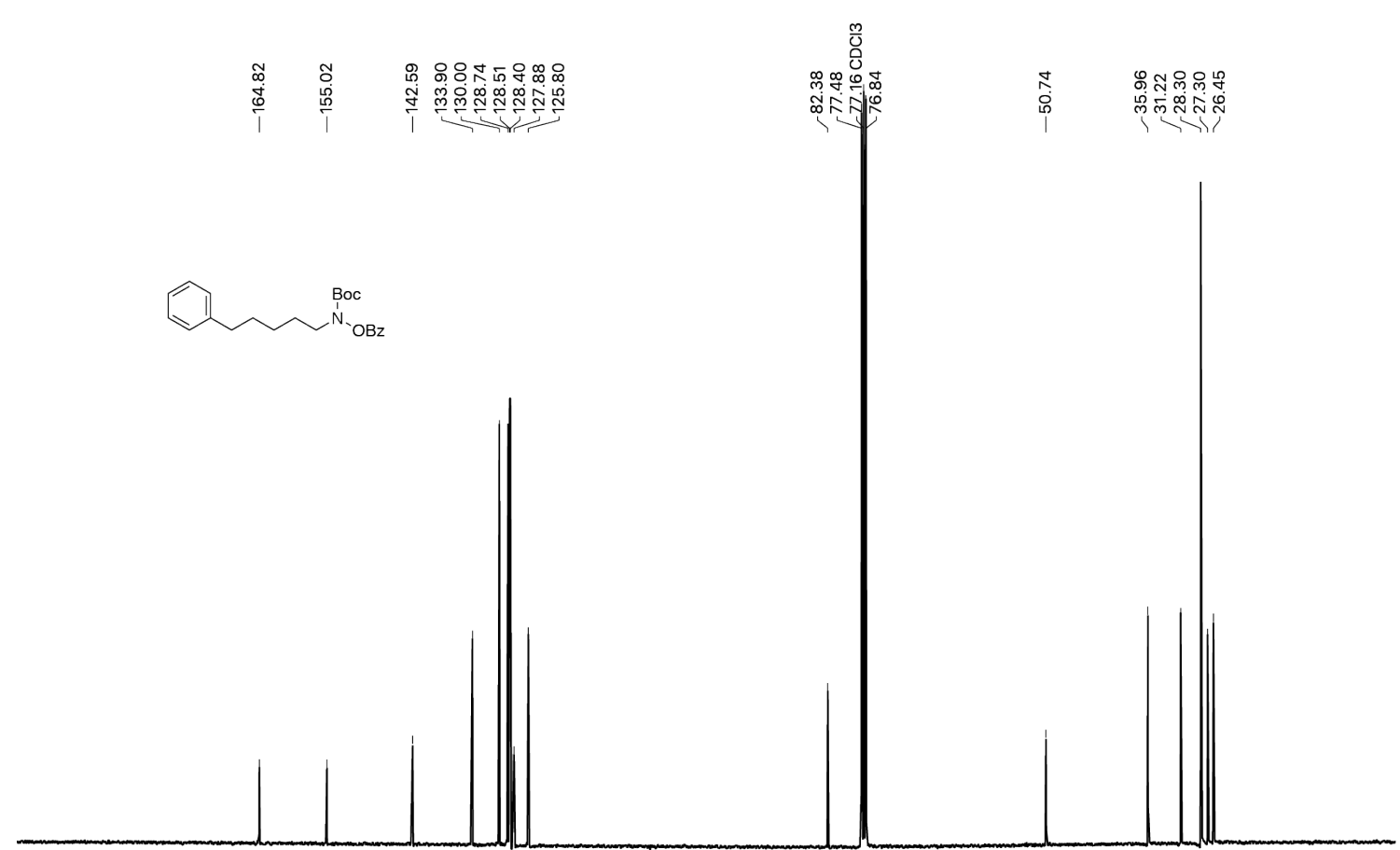

$\begin{array}{llllllll}190 & 180 & 170 & 160 & 150 & 140 & 130 & 120\end{array}$

$110+11000$

Page S34 of S60 
O-Benzoyl- $N$-(5-phenylpentyl)hydroxylamine (1k):

${ }^{1} \mathbf{H}$ NMR (400 MHz, $\left.\mathrm{CDCl}_{3}\right)$
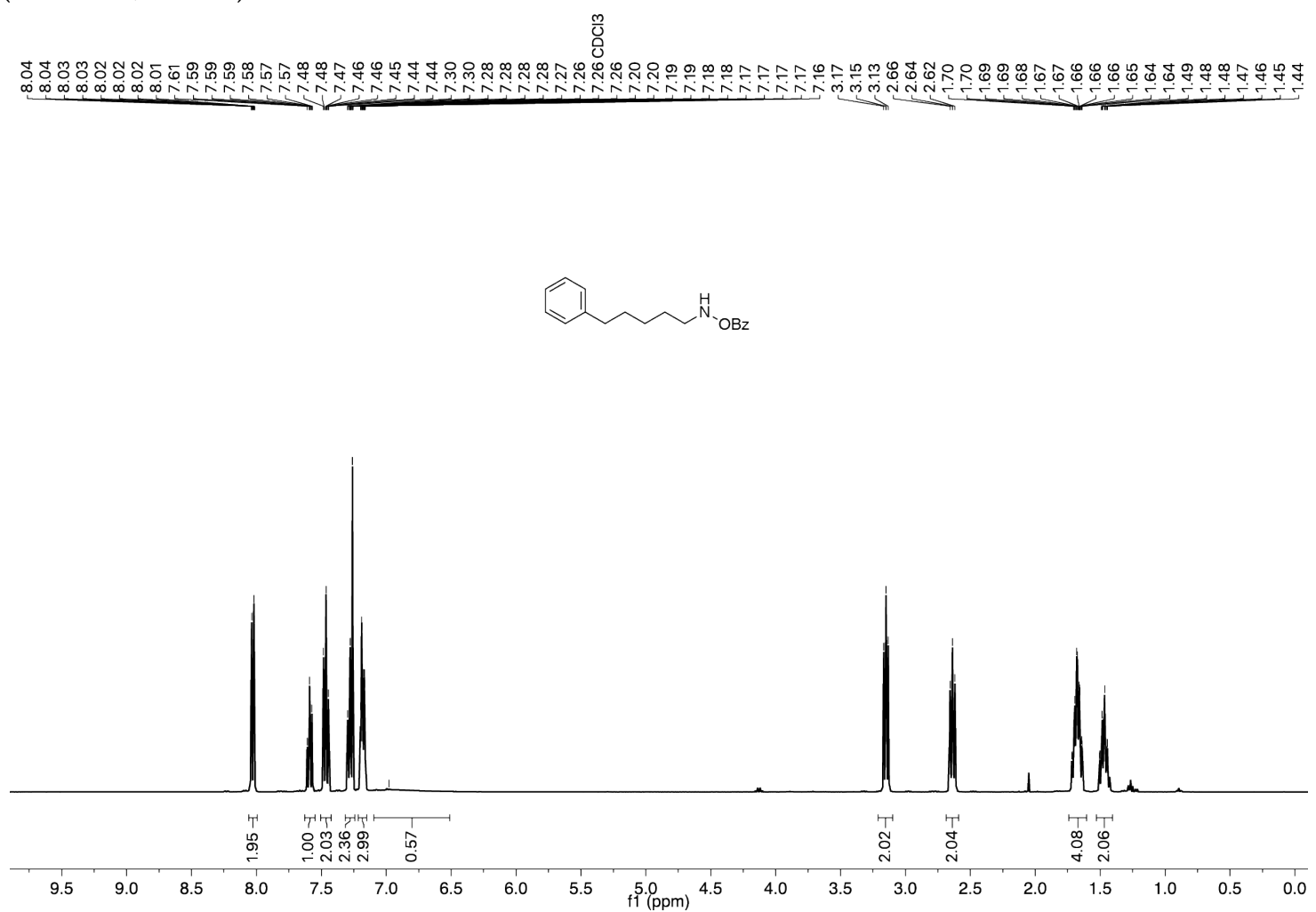

${ }^{13} \mathrm{C}$ NMR (100 MHz, $\left.\mathrm{CDCl}_{3}\right)$

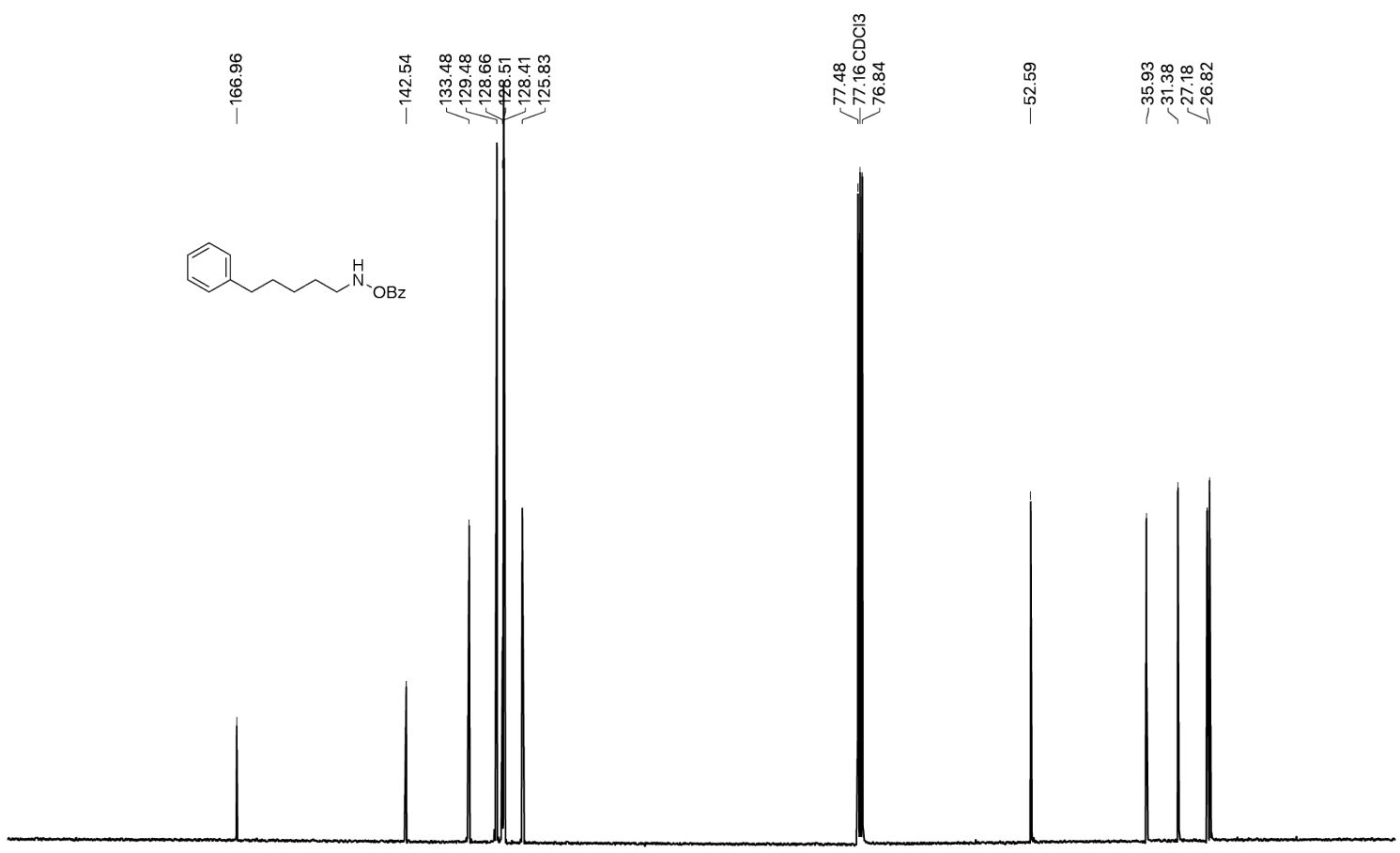

$\begin{array}{llllllll}190 & 180 & 170 & 160 & 150 & 140 & 130 & 120\end{array}$

$110+11000$

8070

Page S35 of S60 
$O$-Benzoyl-N-(((1R,4aS,10aR)-7-isopropyl-1,4a-dimethyl-1,2,3,4,4a,9,10,10a-octahydrophenanthren-1yl)methyl)hydroxylamine (1m):

${ }^{1} \mathbf{H}$ NMR $\left(400 \mathrm{MHz}, \mathrm{CDCl}_{3}\right)$
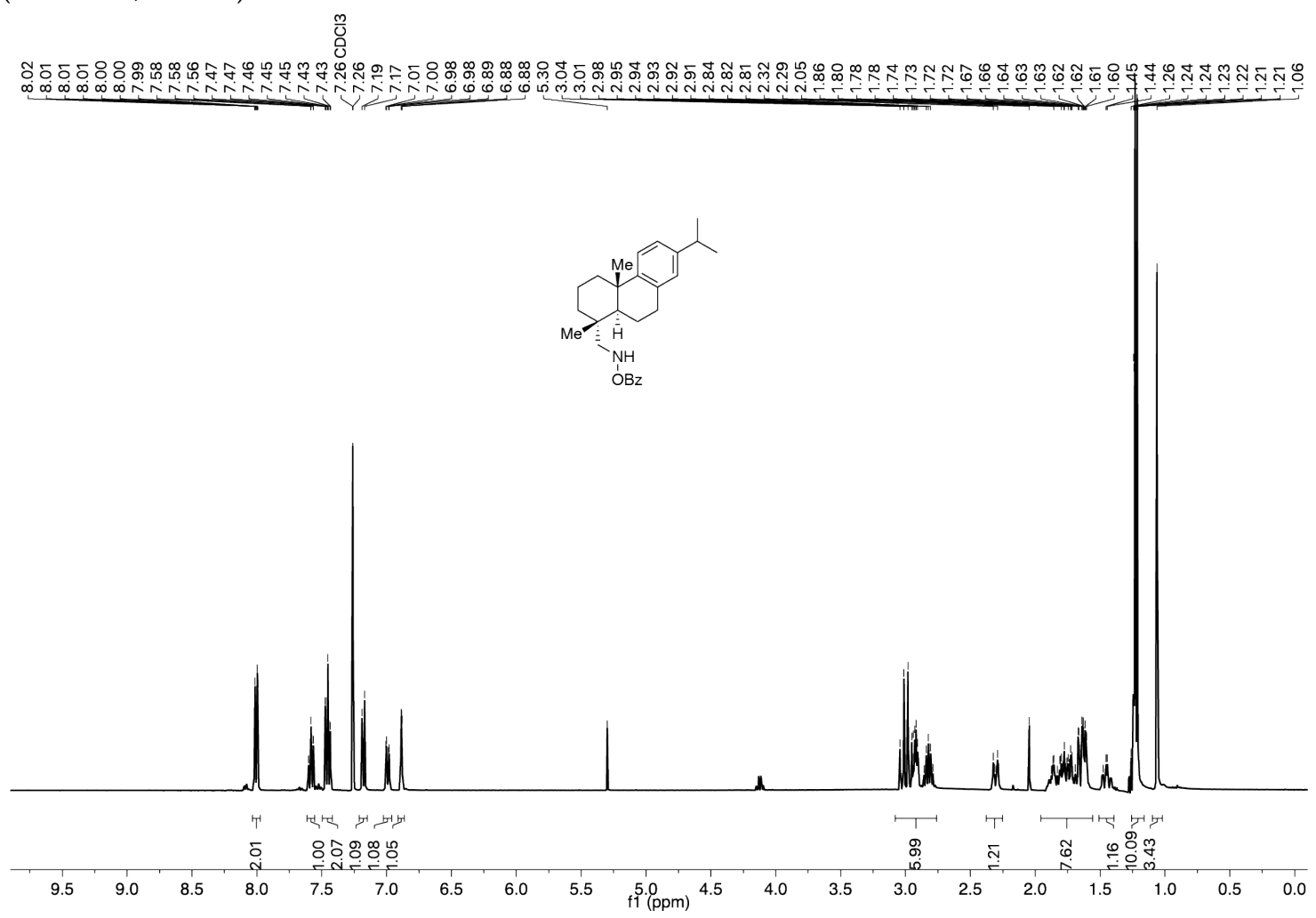

${ }^{13} \mathrm{C}$ NMR $\left(100 \mathrm{MHz}, \mathrm{CDCl}_{3}\right)$
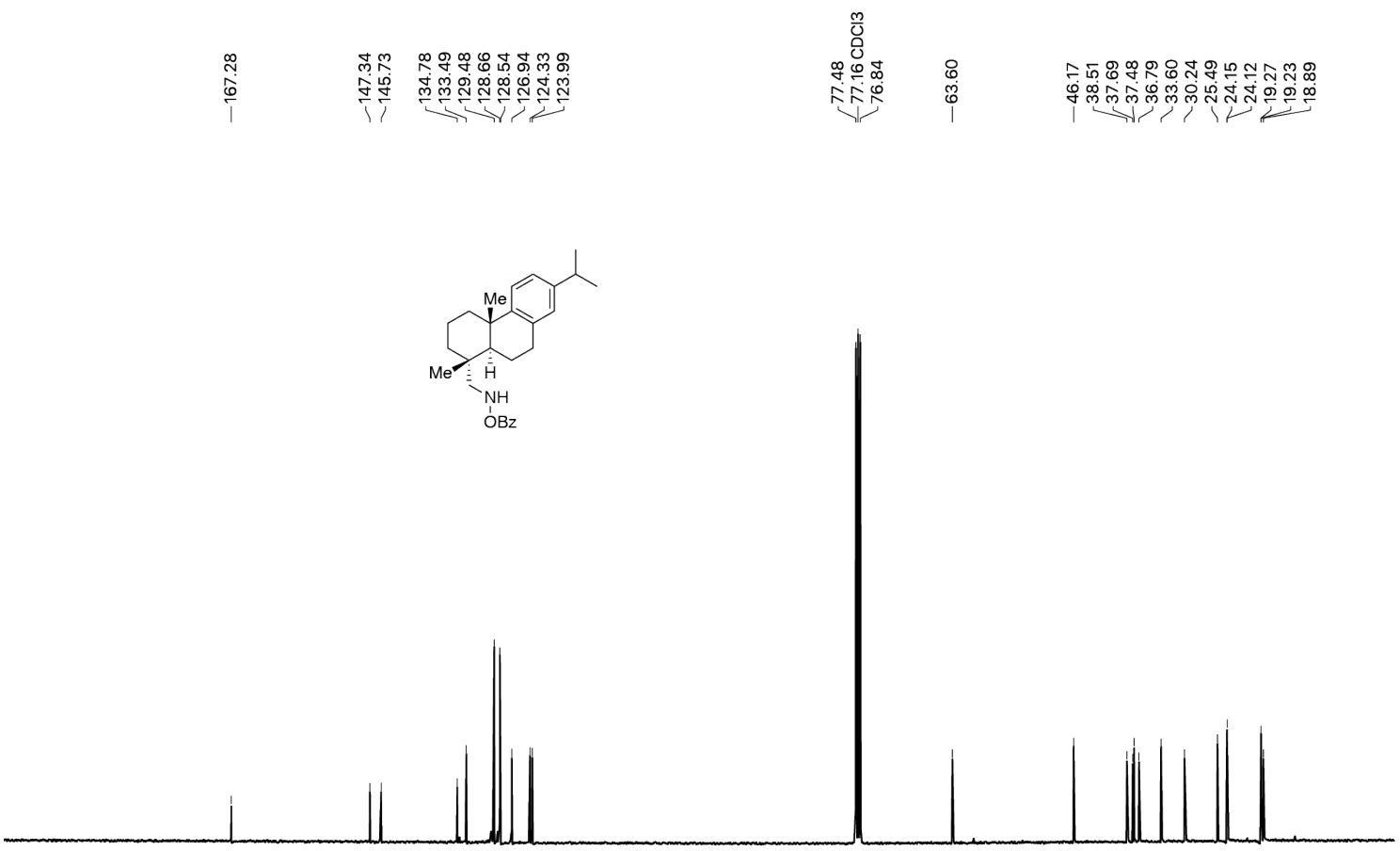

$\begin{array}{llllllll}190 & 180 & 170 & 160 & 150 & 140 & 130 & 120\end{array}$ Page S36 of S60 
tert-Butyl (benzoyloxy)(4-(thiophen-3-yl)butyl)carbamate (Boc-S1):

${ }^{1} \mathbf{H}$ NMR (400 MHz, $\left.\mathrm{CDCl}_{3}\right)$
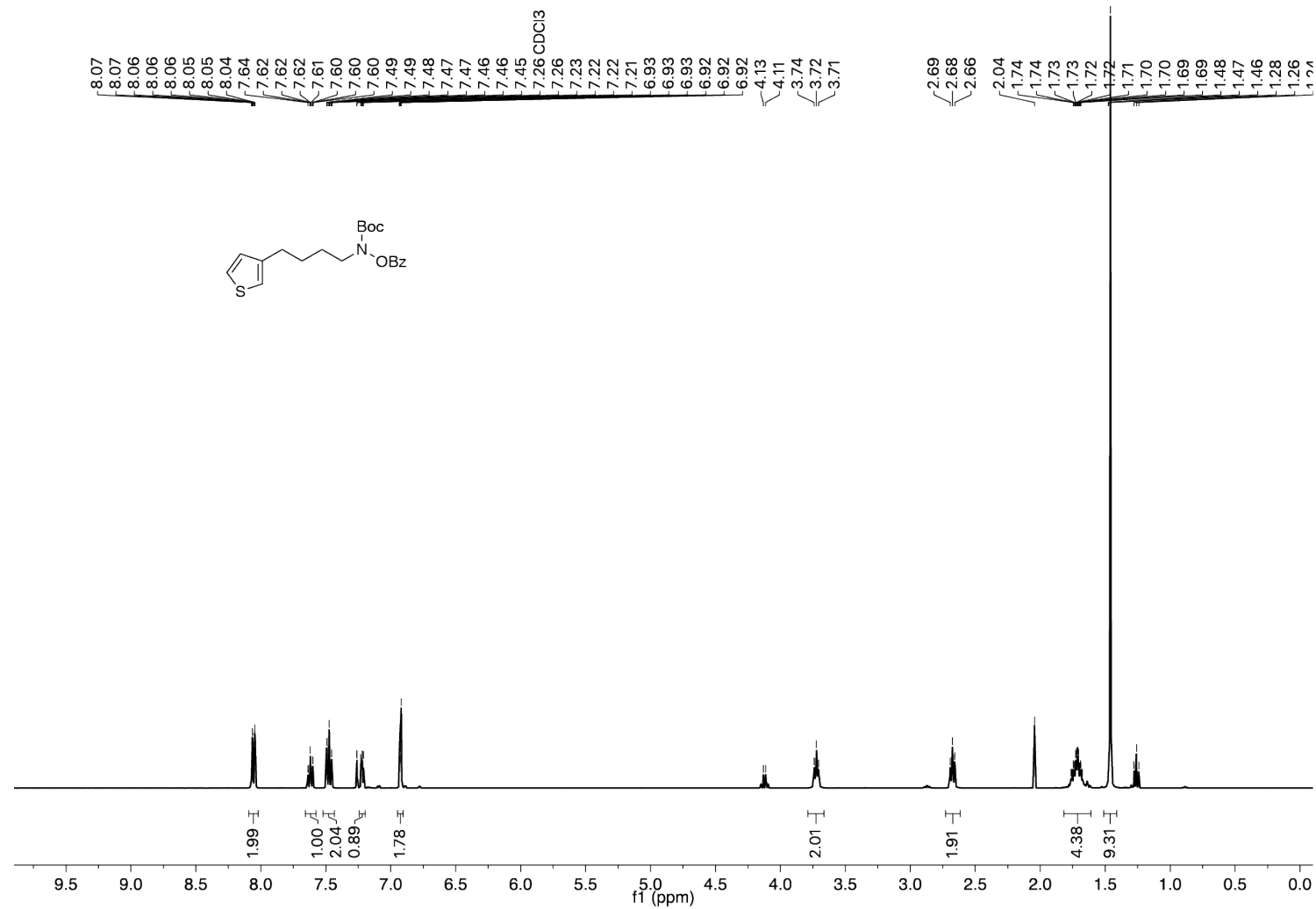

${ }^{13} \mathrm{C}$ NMR $\left(100 \mathrm{MHz}, \mathrm{CDCl}_{3}\right)$
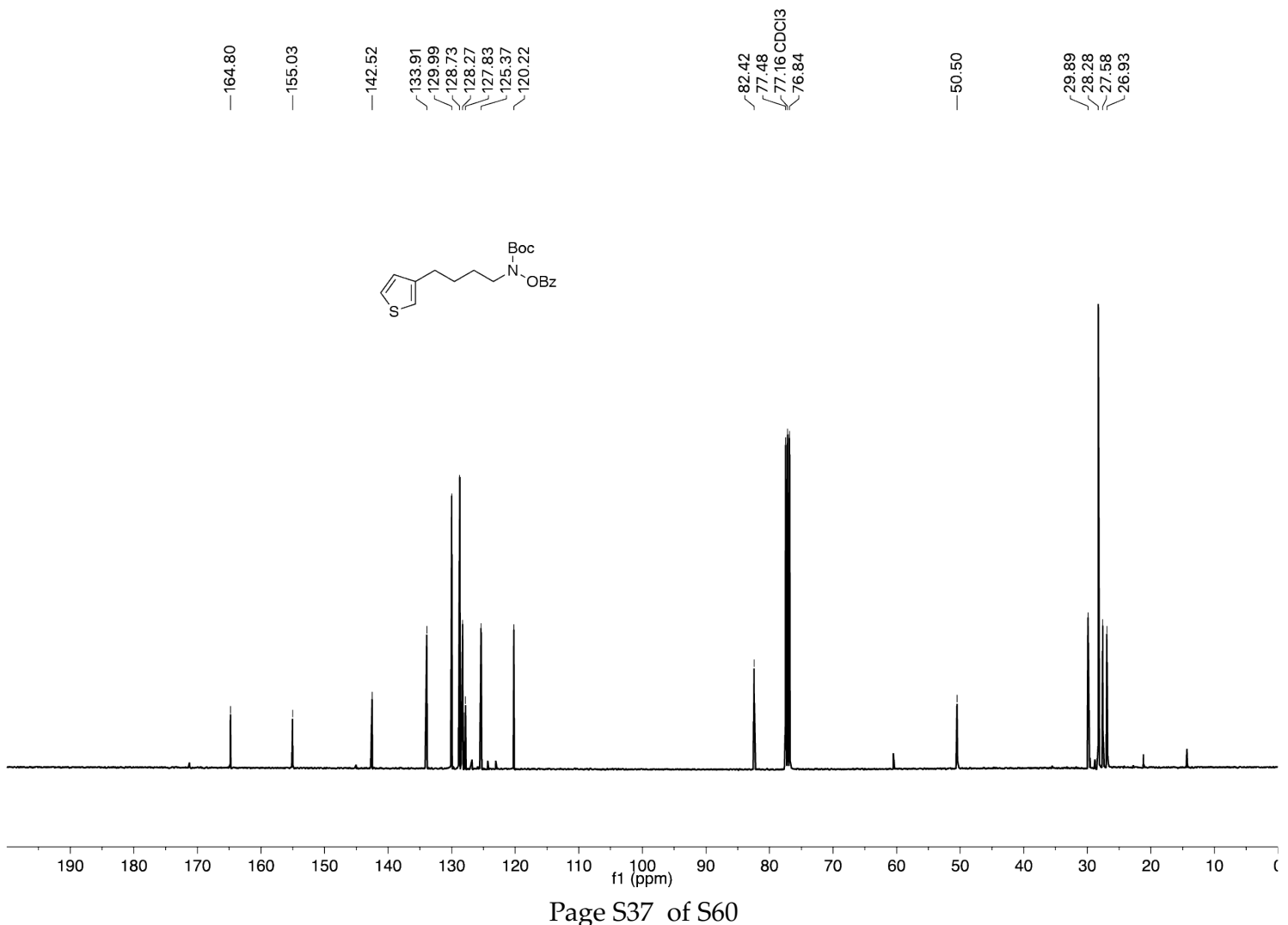

Page S37 of S60 
O-Benzoyl-N-(4-(thiophen-3-yl)butyl)hydroxylamine (S1):

${ }^{1} \mathbf{H}$ NMR (400 MHz, $\left.\mathrm{CDCl}_{3}\right)$
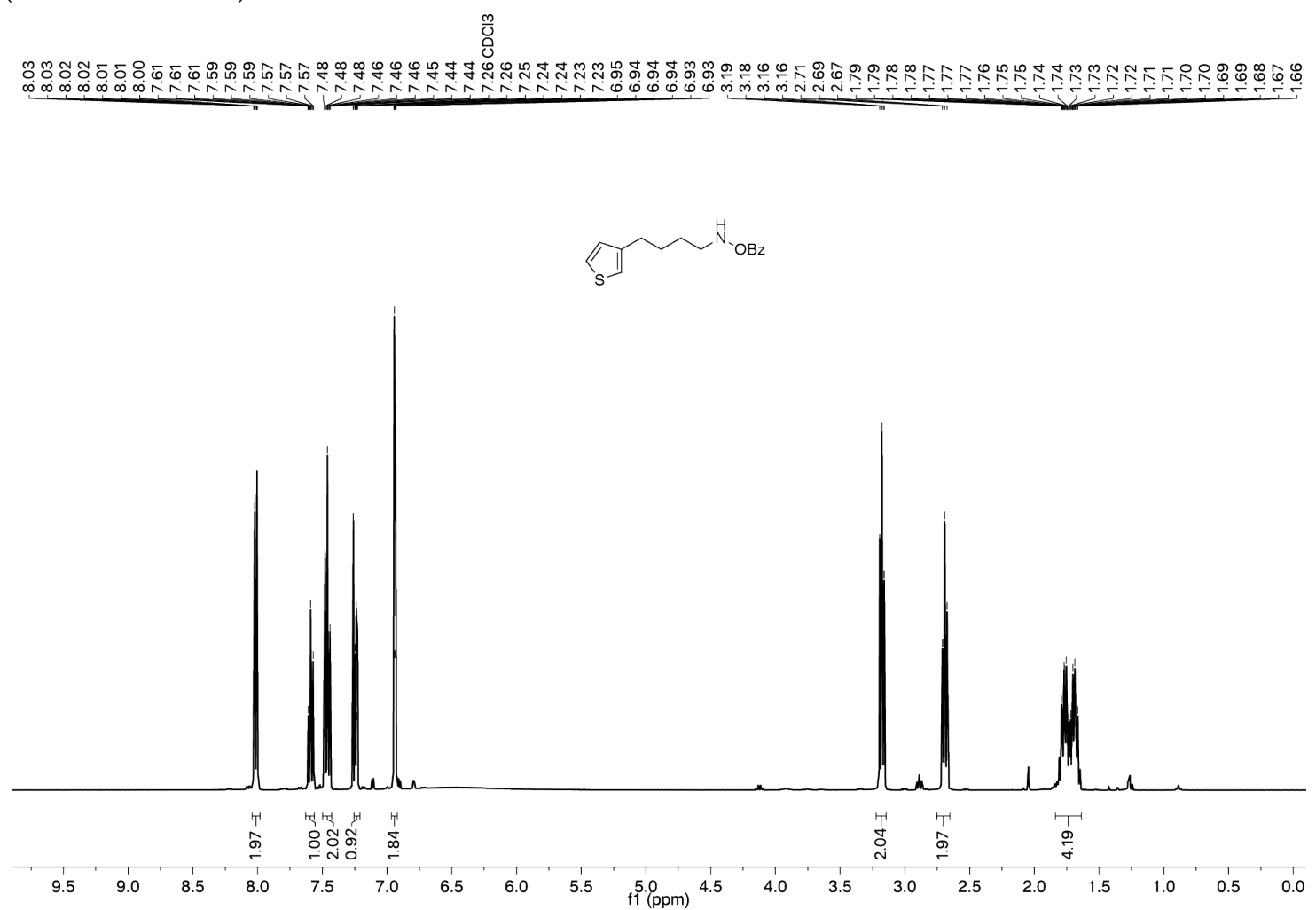

${ }^{13} \mathrm{C}$ NMR (100 MHz, $\left.\mathrm{CDCl}_{3}\right)$
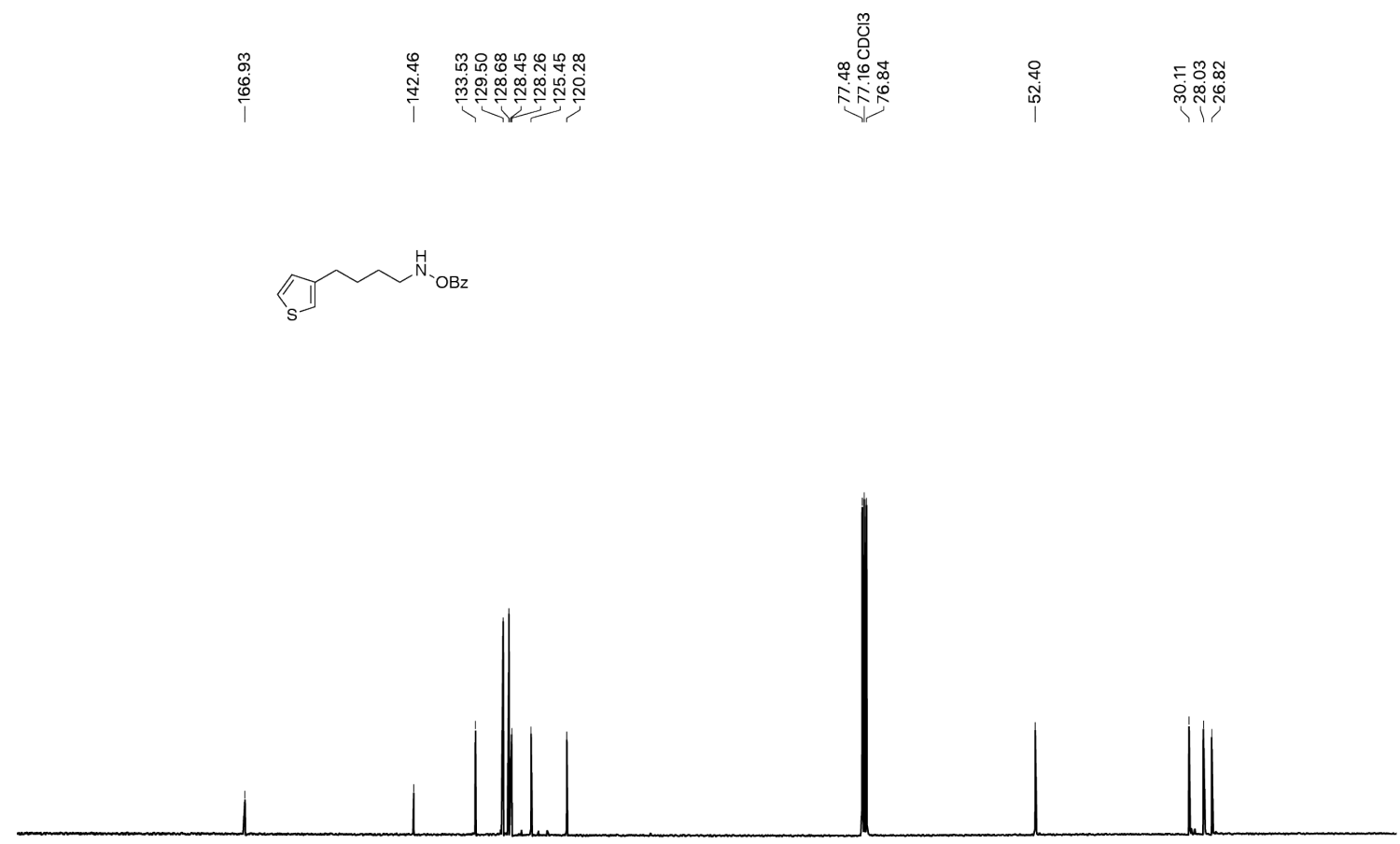

$\begin{array}{llllllll}190 & 180 & 170 & 160 & 150 & 140 & 130 & 120\end{array}$

$110+11000$

$80 \quad 70$

Page S38 of S60 
O-Benzoyl-N-(4-(pyridin-4-yl)butyl)hydroxylamine (S2):

${ }^{1} \mathbf{H}$ NMR (400 MHz, $\mathrm{CDCl}_{3}$ )
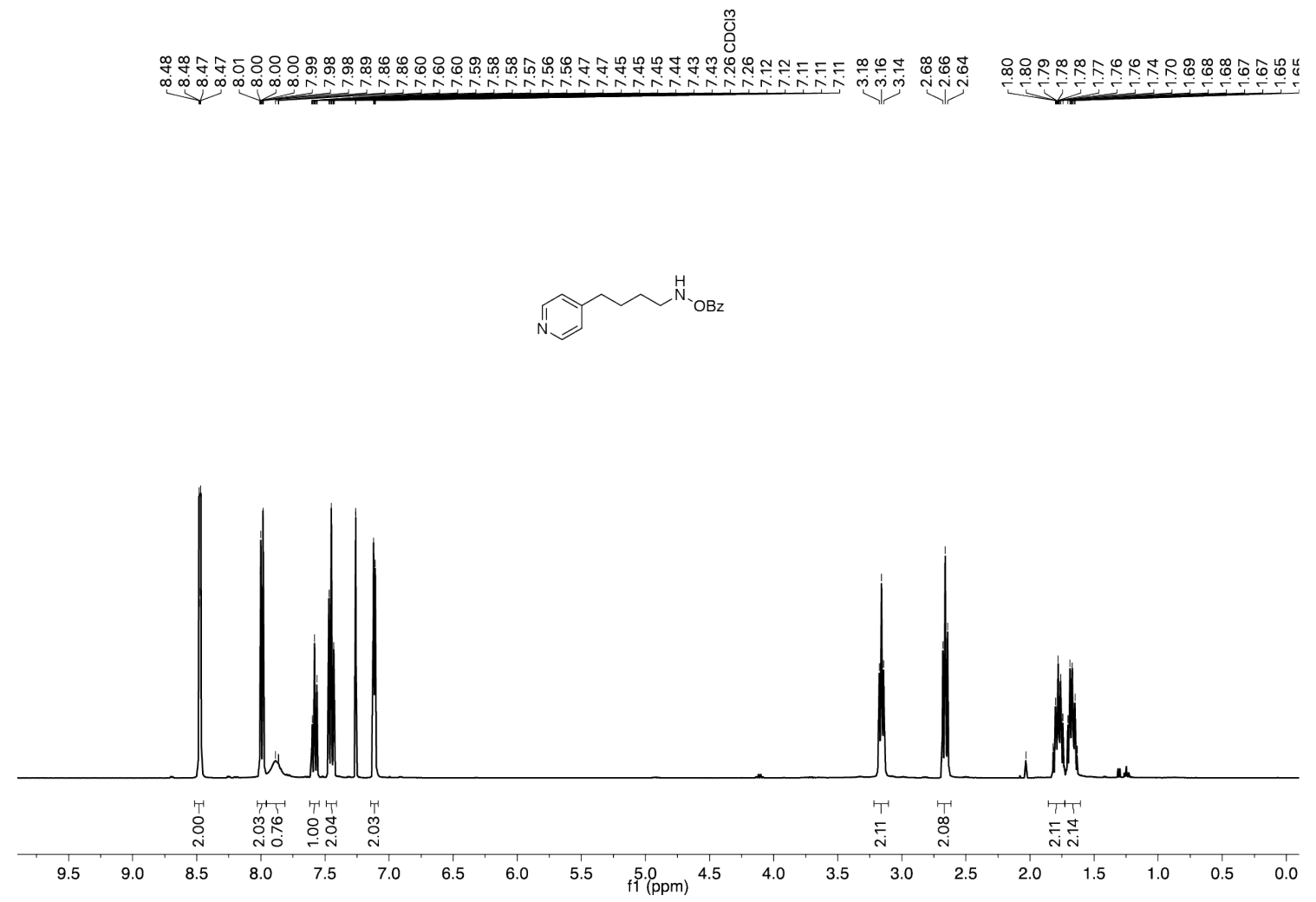

${ }^{13} \mathrm{C}$ NMR (100 MHz, $\left.\mathrm{CDCl}_{3}\right)$

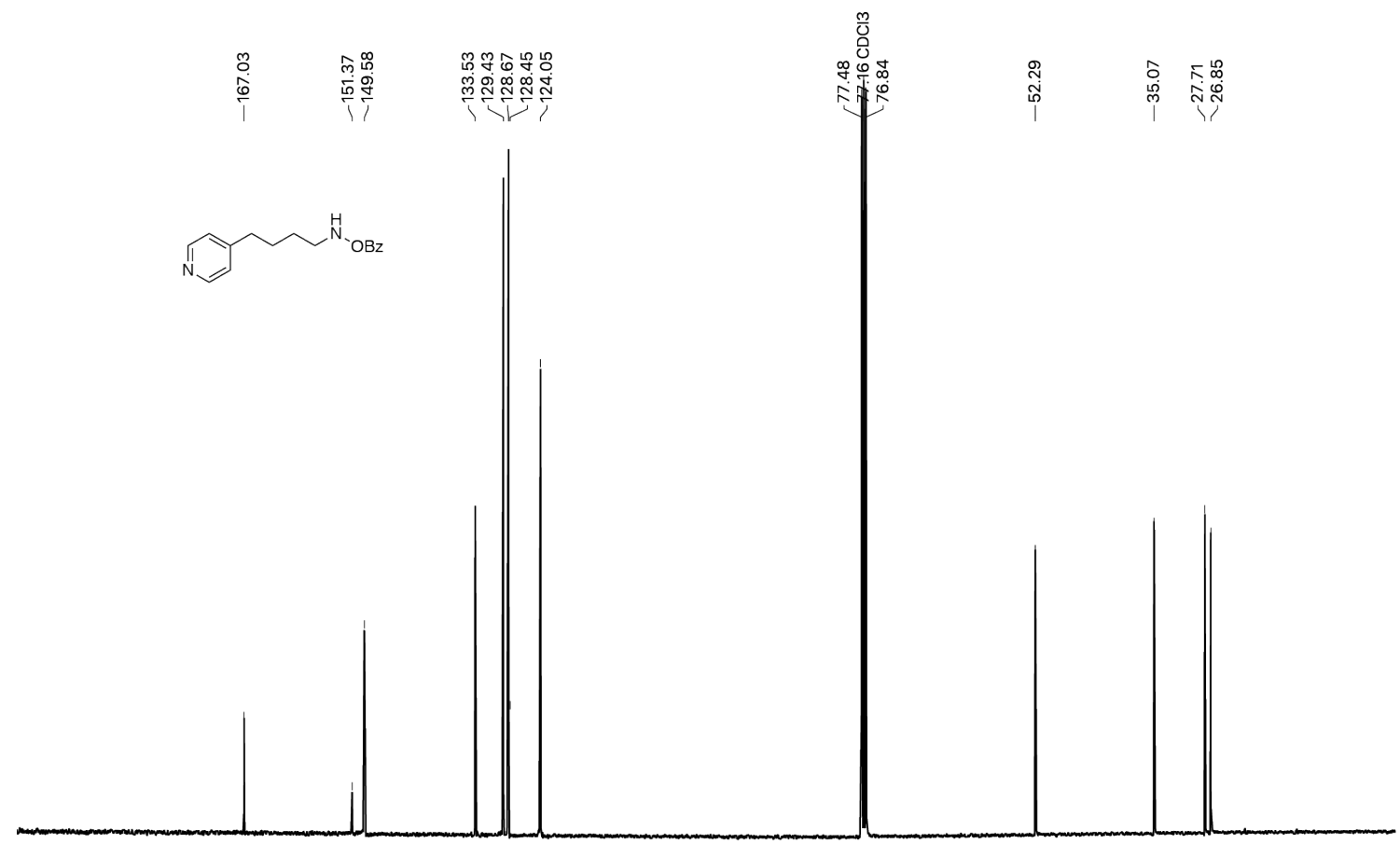

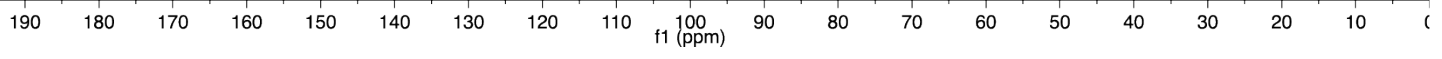

Page S39 of S60 
$N$-Boc 2-phenylpyrrolidine (Boc-2a):

${ }^{1} \mathbf{H}$ NMR (400 MHz, $\mathrm{CDCl}_{3}$, rotamer ratio 30:70)
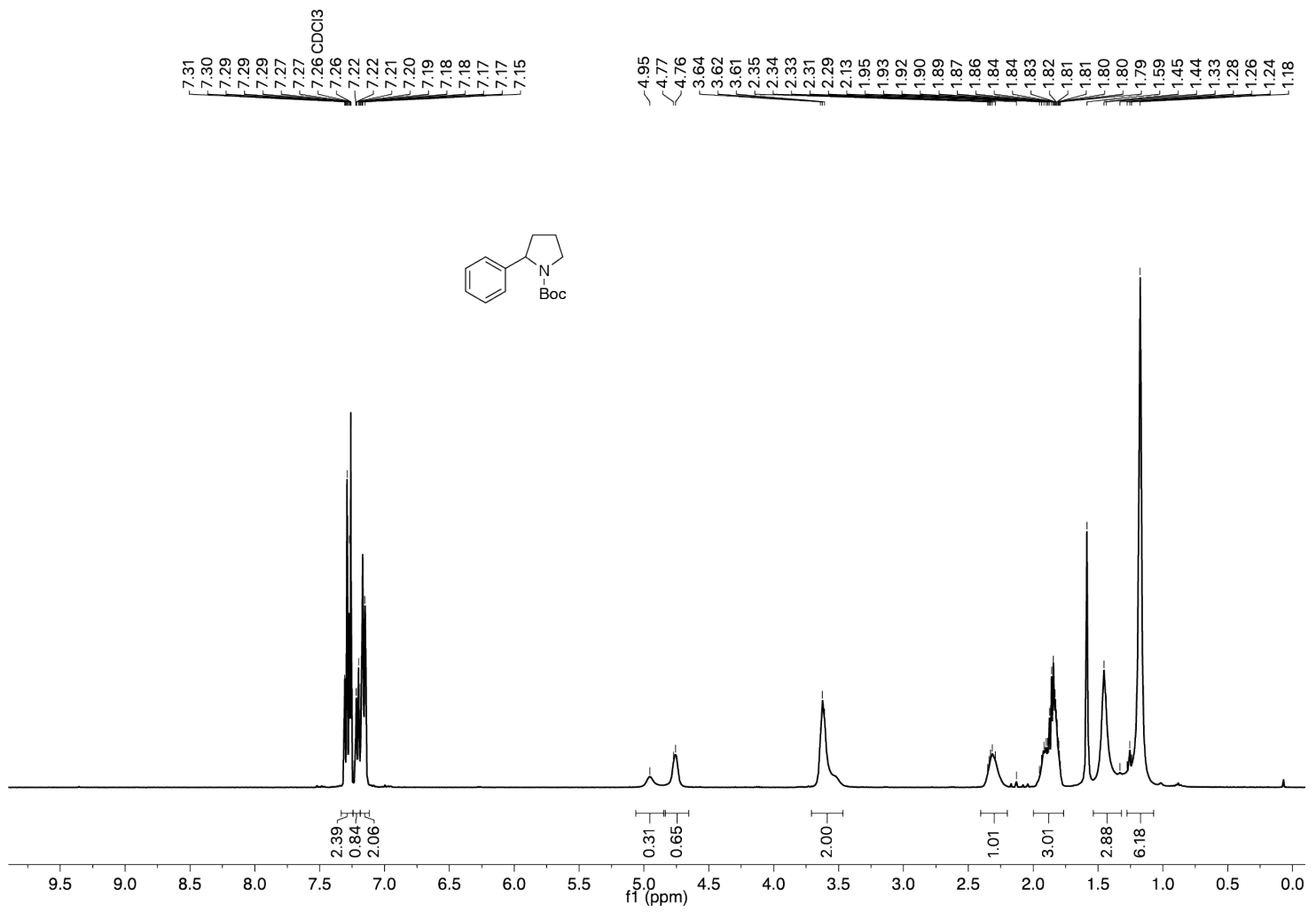
$N$-Boc 2-( $p$-tolyl)pyrrolidine (Boc-2b):

${ }^{1} \mathbf{H}$ NMR (400 MHz, $\mathrm{CDCl}_{3}$, rotamer ratio 30:70)
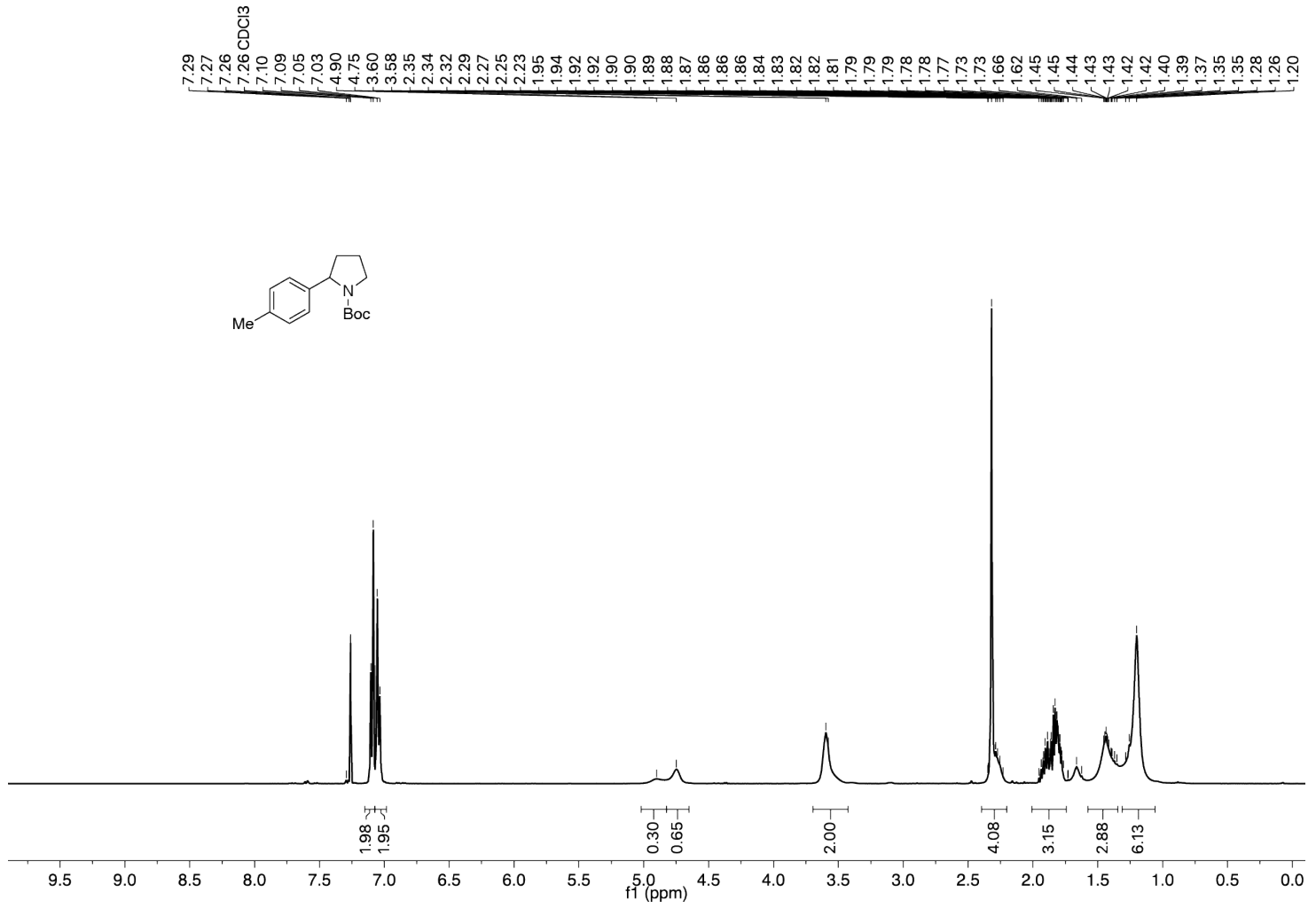
N-Boc 2-(4-methoxyphenyl)pyrrolidine (Boc-2c):

${ }^{1} \mathbf{H}$ NMR (400 MHz, $\mathrm{CDCl}_{3}$, rotamer ratio 30:70)

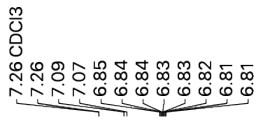

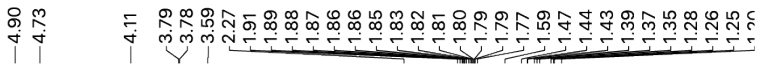
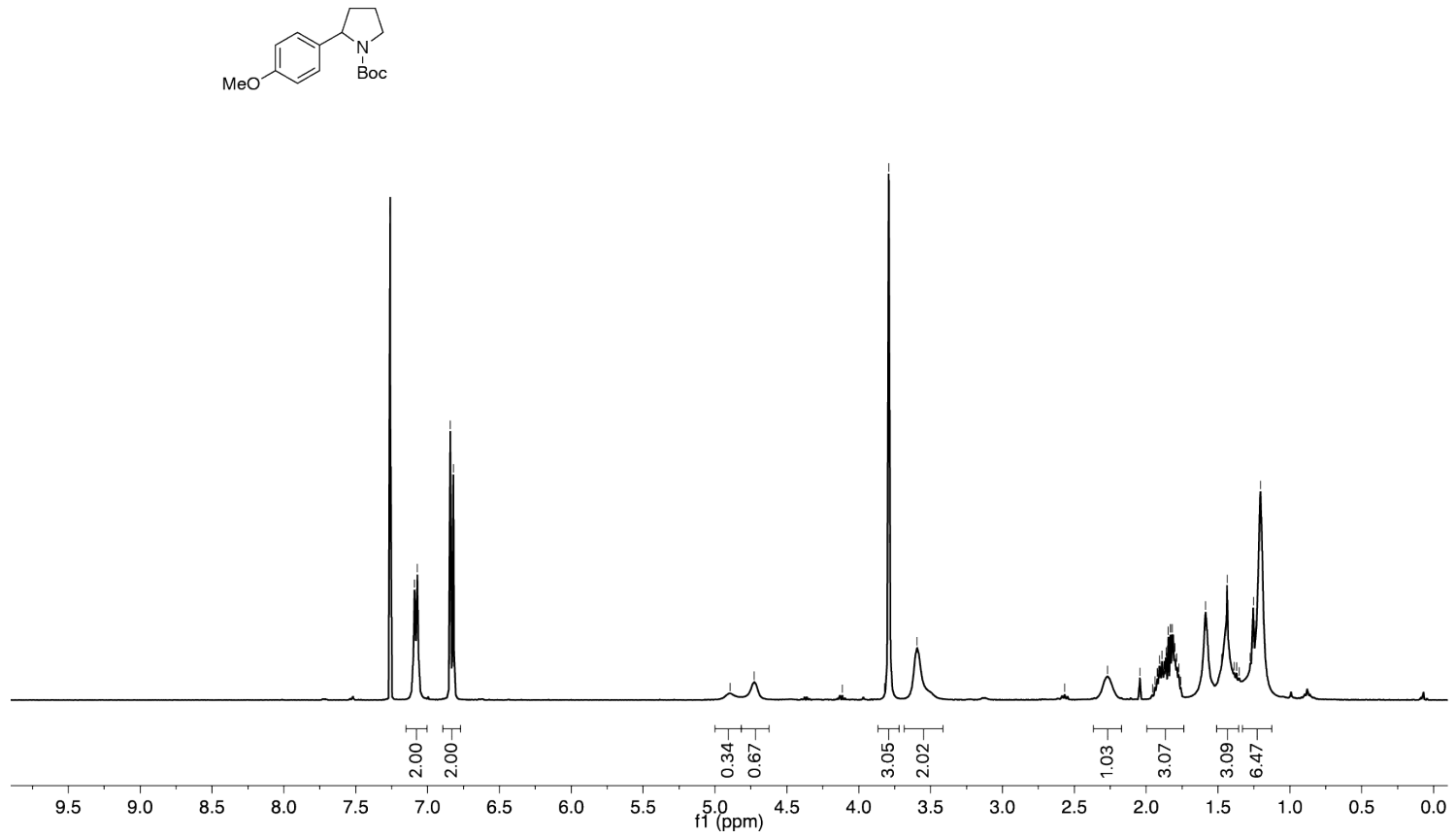
N-Boc 4-(pyrrolidin-2-yl)benzonitrile (Boc-2d):

${ }^{1} \mathbf{H}$ NMR (400 MHz, $\mathrm{CDCl}_{3}$, rotamer ratio 30:70)
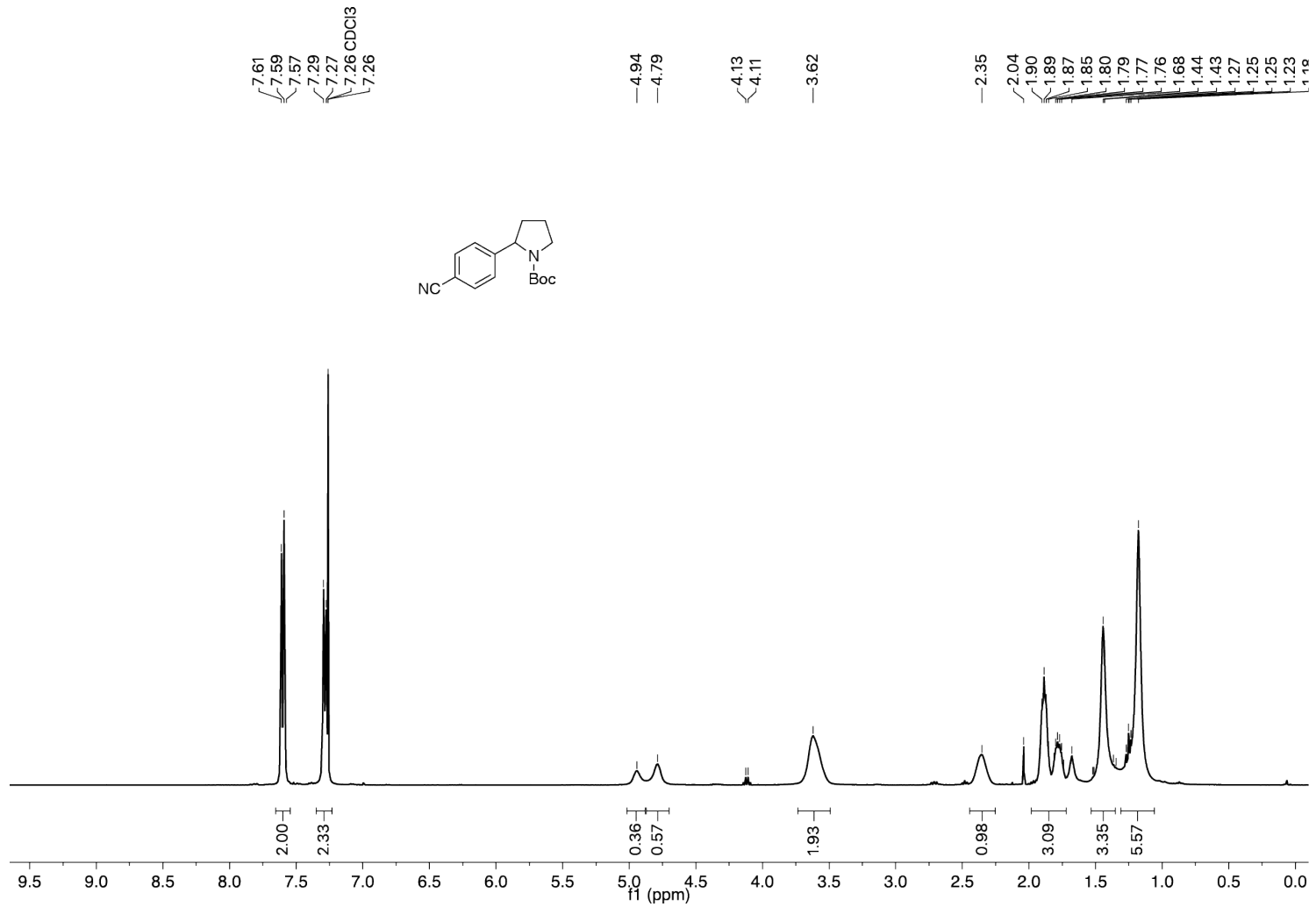
$\mathrm{N}$-Boc methyl 4-(pyrrolidin-2-yl)benzoate (Boc-2e):

${ }^{1} \mathbf{H}$ NMR (400 MHz, $\mathrm{CDCl}_{3}$, rotamer ratio 30:70)
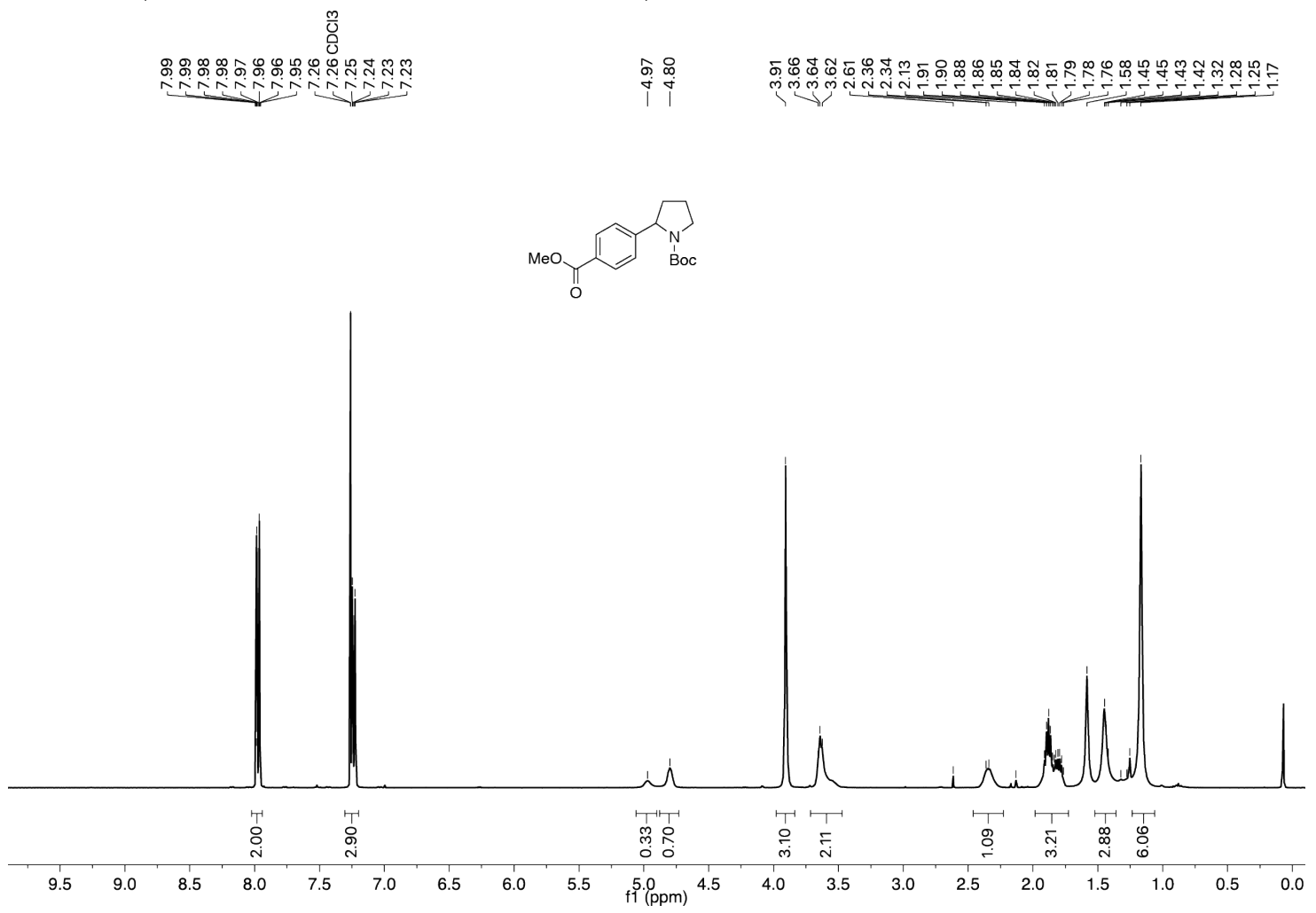
N-Boc 2-(3-methoxyphenyl)pyrrolidine (Boc-2f):

${ }^{1} \mathbf{H}$ NMR (400 MHz, $\mathrm{CDCl}_{3}$, rotamer ratio 30:70)

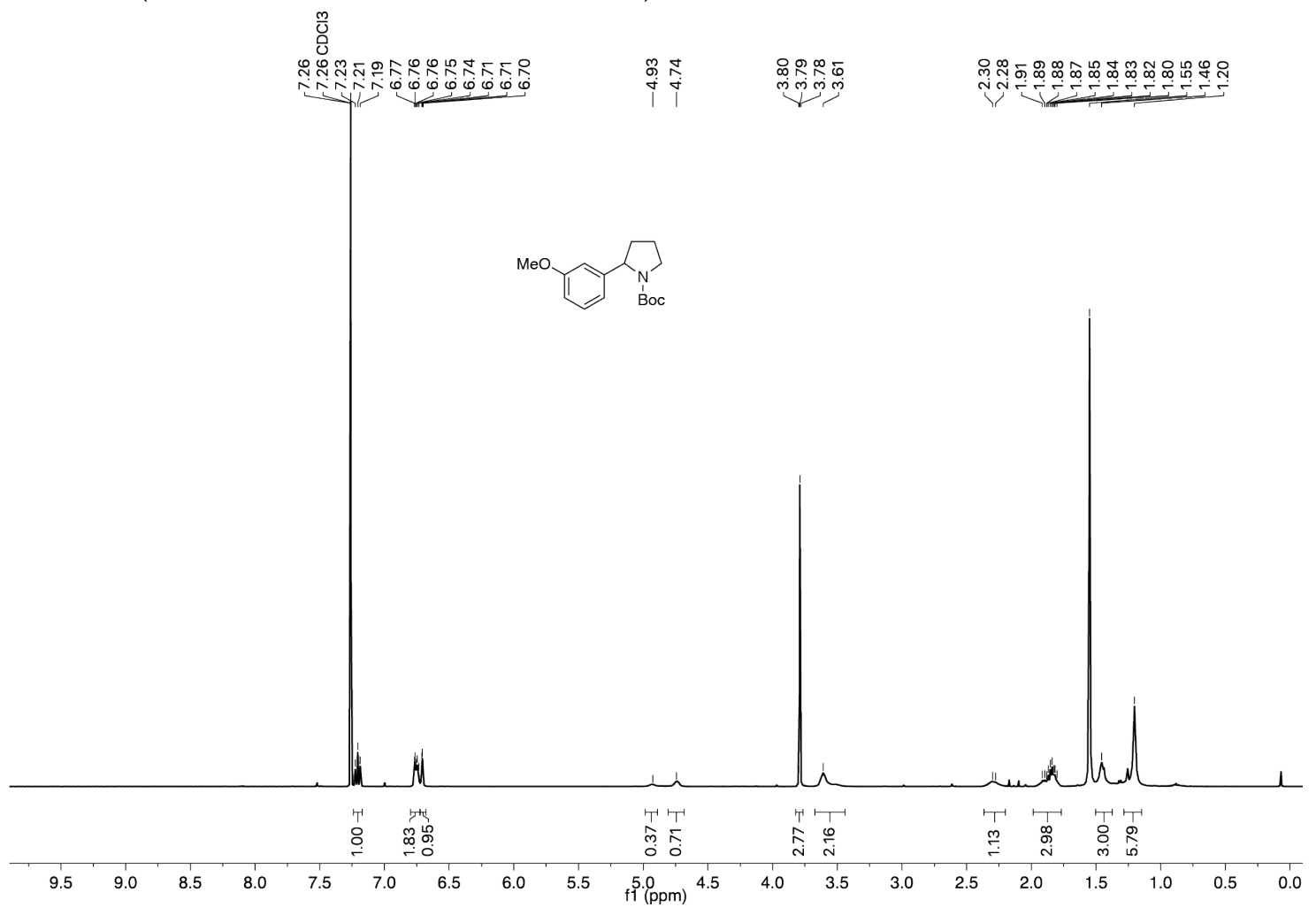


$\mathrm{N}$-Boc methyl 2-(pyrrolidin-2-yl)benzoate (Boc-2g):

${ }^{1} \mathbf{H}$ NMR (400 MHz, $\mathrm{CDCl}_{3}$, rotamer ratio 30:70)

O․

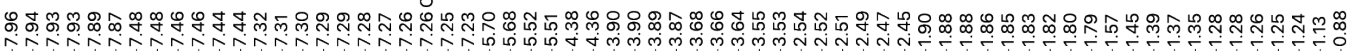

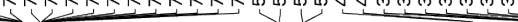

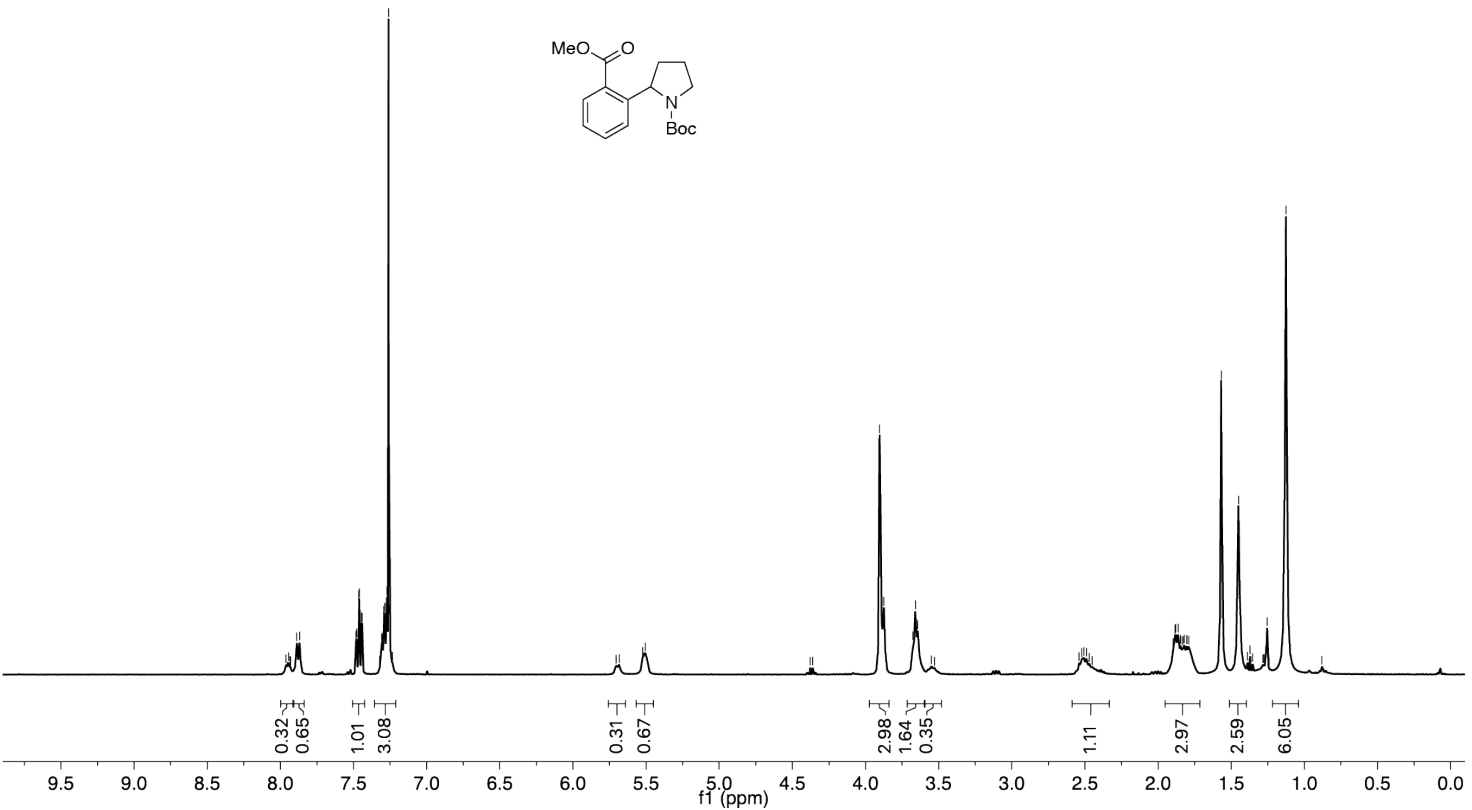


$N$-Boc 2,2,5-trimethylpyrrolidine (Boc-2i):

${ }^{1} \mathbf{H}$ NMR $\left(400 \mathrm{MHz}, \mathrm{CDCl}_{3}\right)$

思

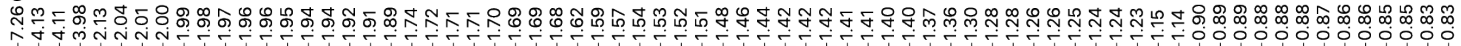

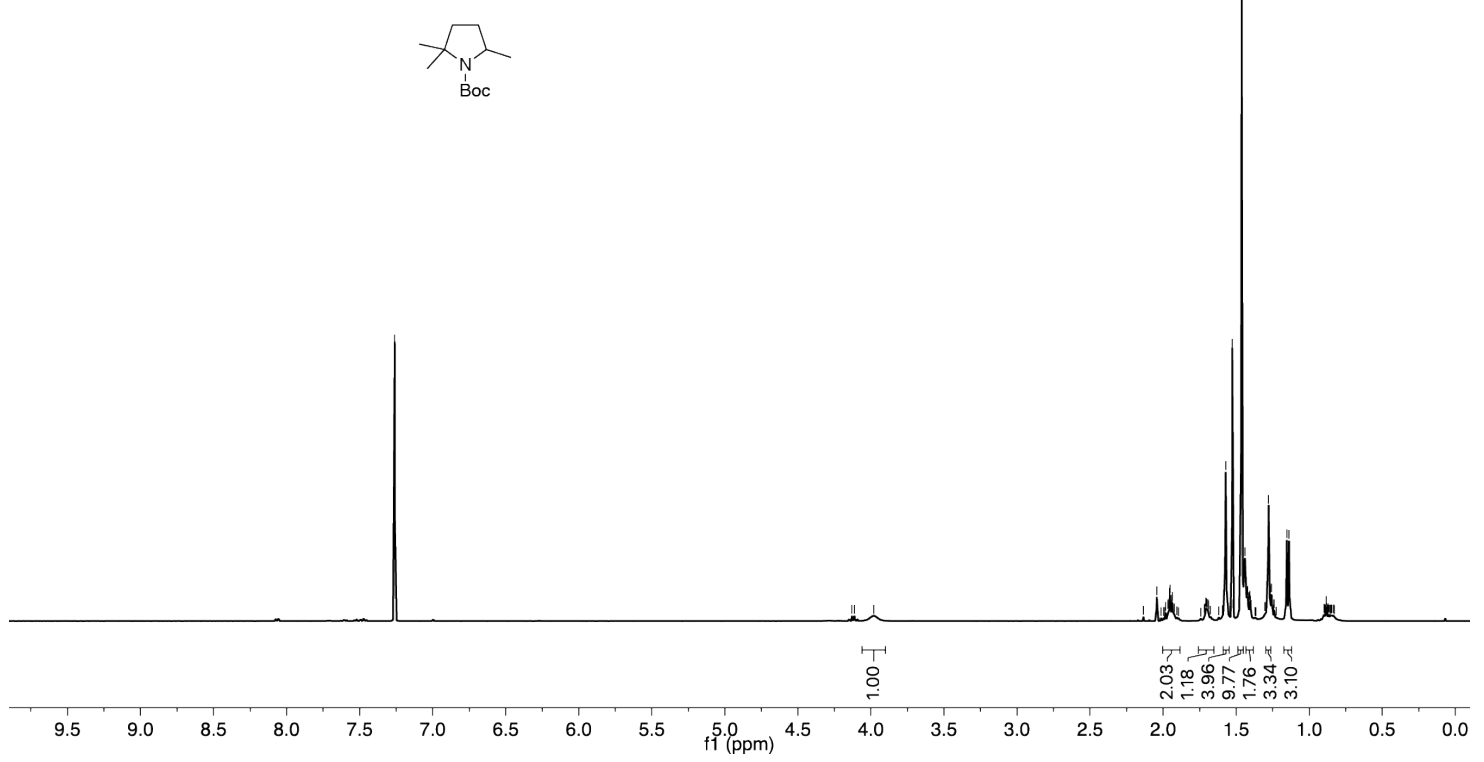

${ }^{13} \mathrm{C}$ NMR $\left(100 \mathrm{MHz}, \mathrm{CDCl}_{3}\right)$

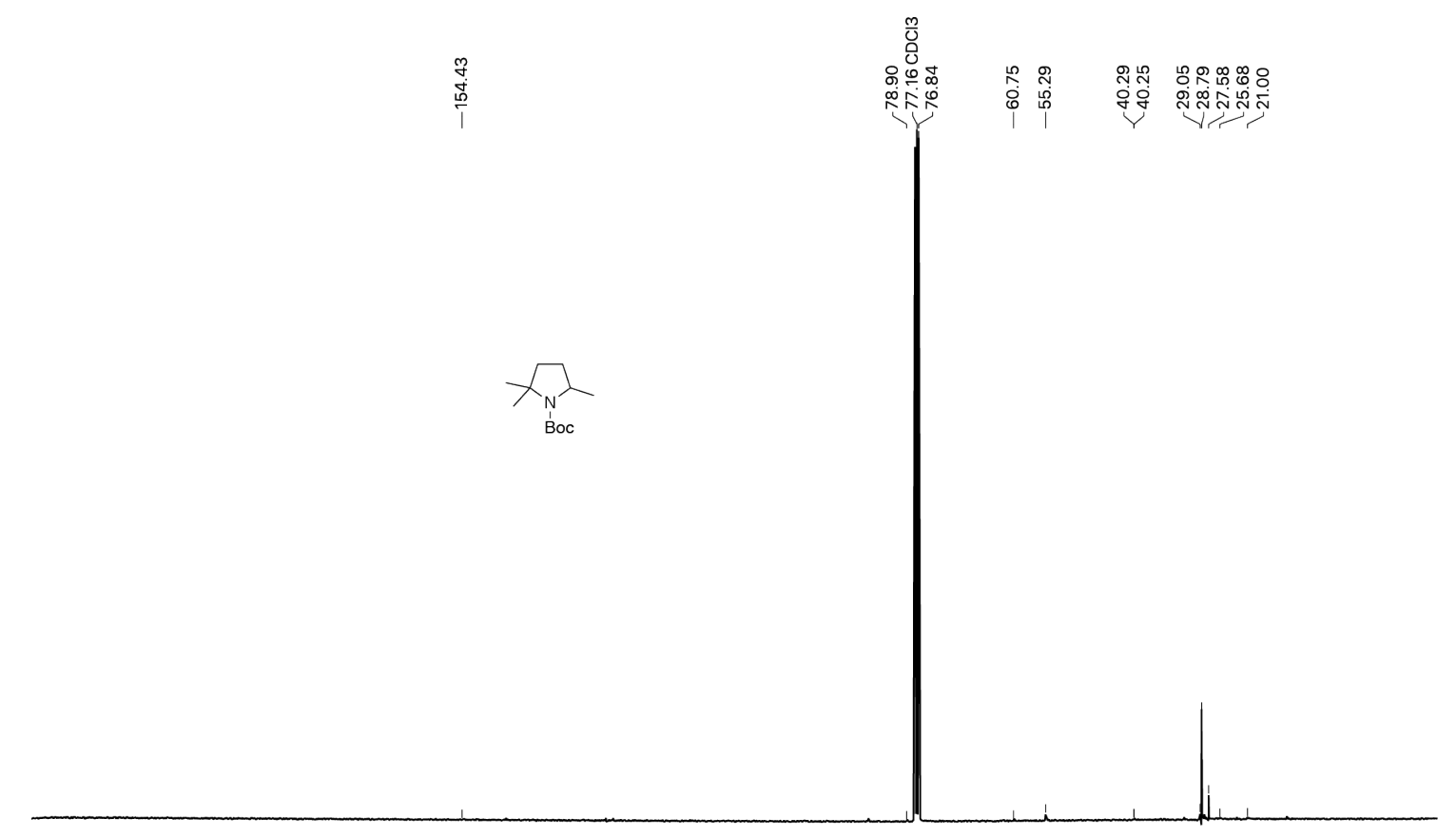

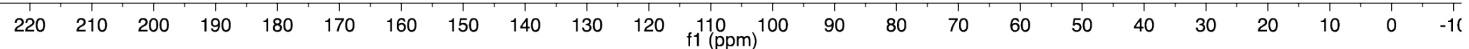


$\mathrm{N}$-Boc 2-propylpyrrolidine (Boc-2j):

${ }^{1} \mathbf{H}$ NMR $\left(400 \mathrm{MHz}, \mathrm{CDCl}_{3}\right)$
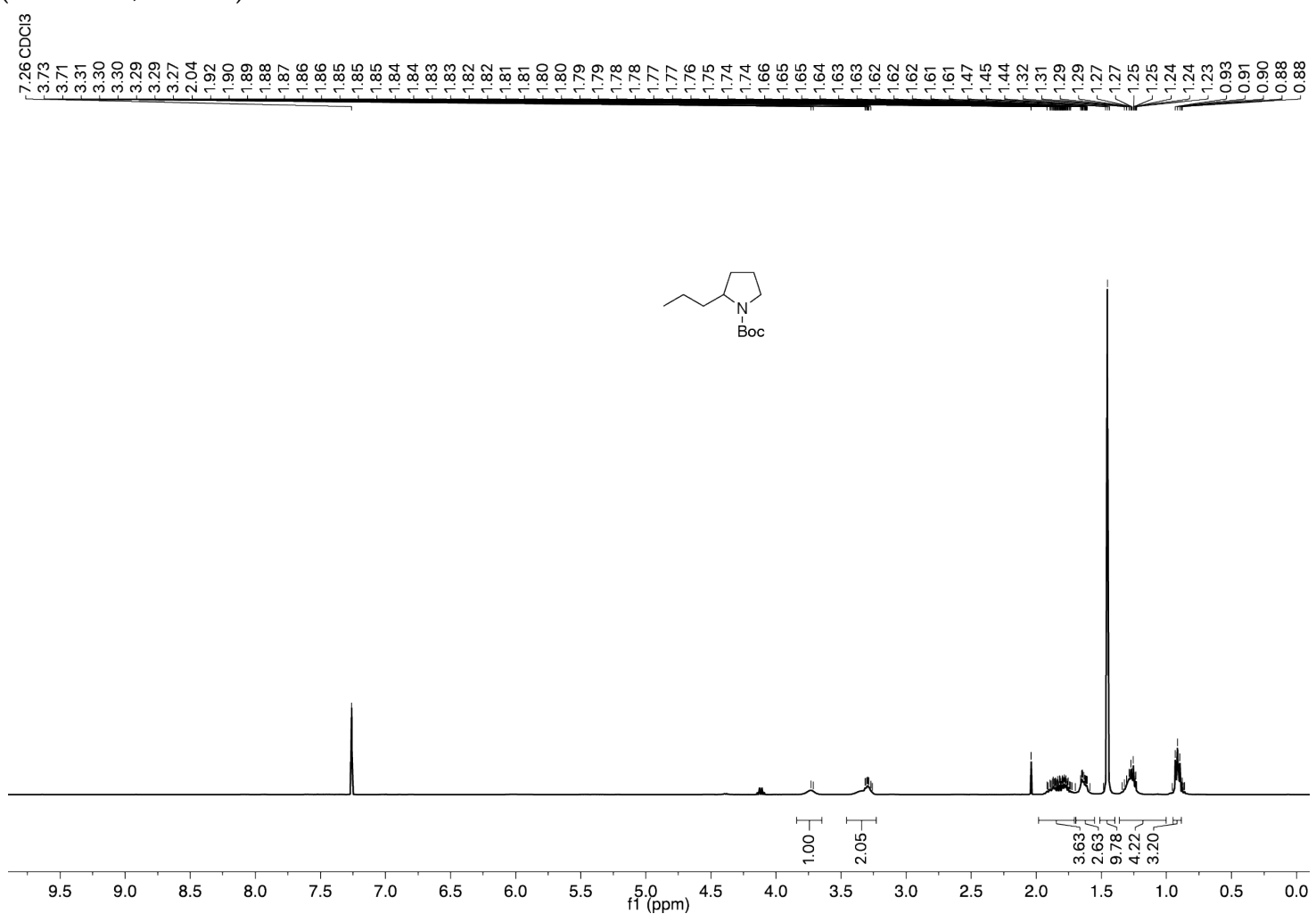

${ }^{13} \mathrm{C}$ NMR (100 MHz, $\left.\mathrm{CDCl}_{3}\right)$

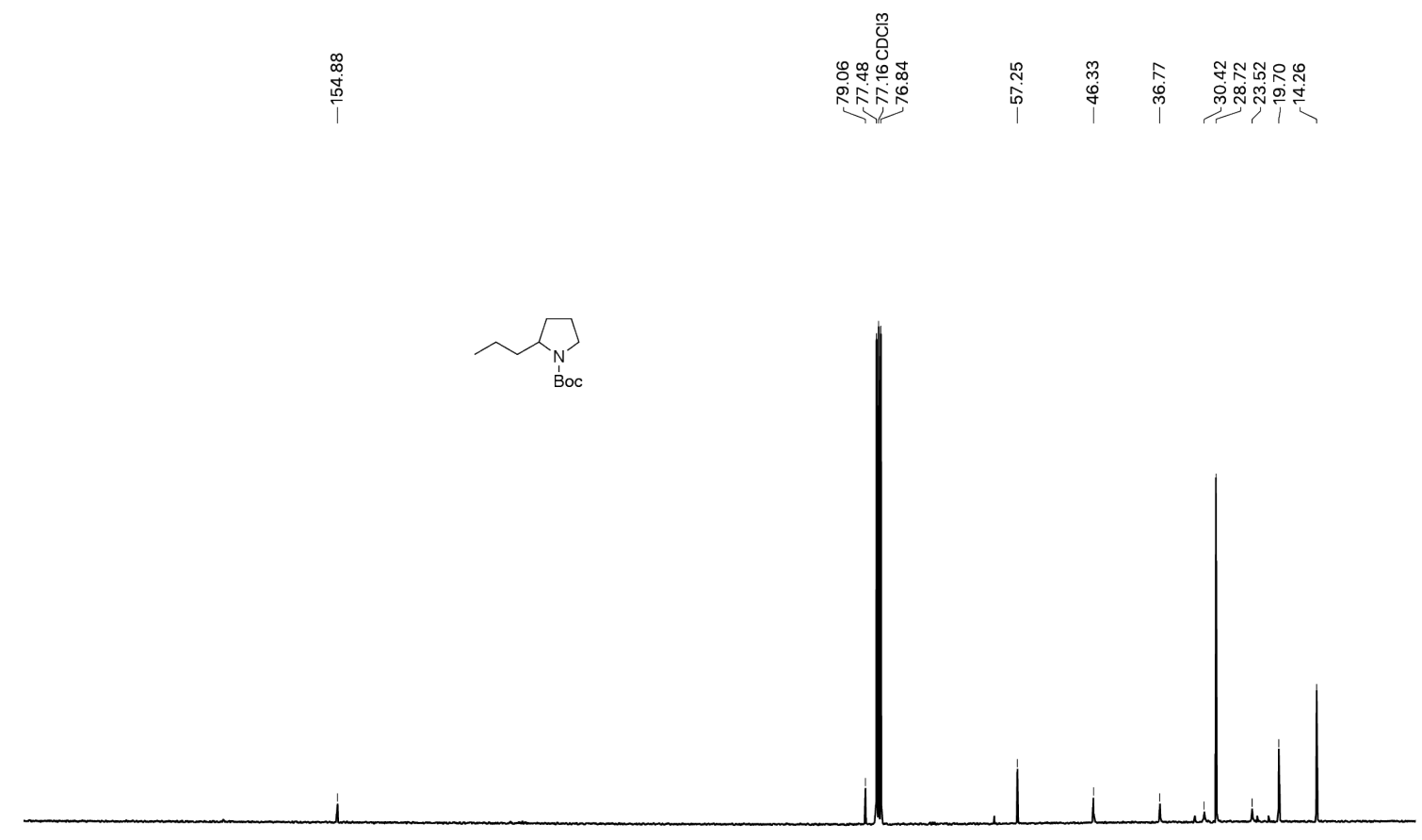

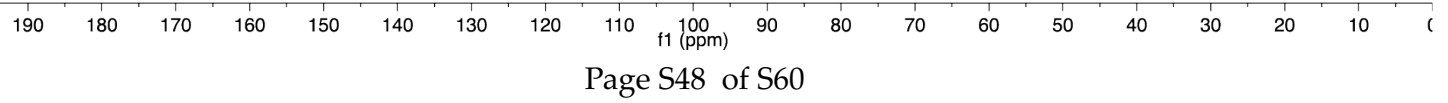


N-Boc 2-benzylpyrrolidine (Boc-2k):

${ }^{1} \mathbf{H}$ NMR (400 MHz, $\mathrm{CDCl}_{3}$ )
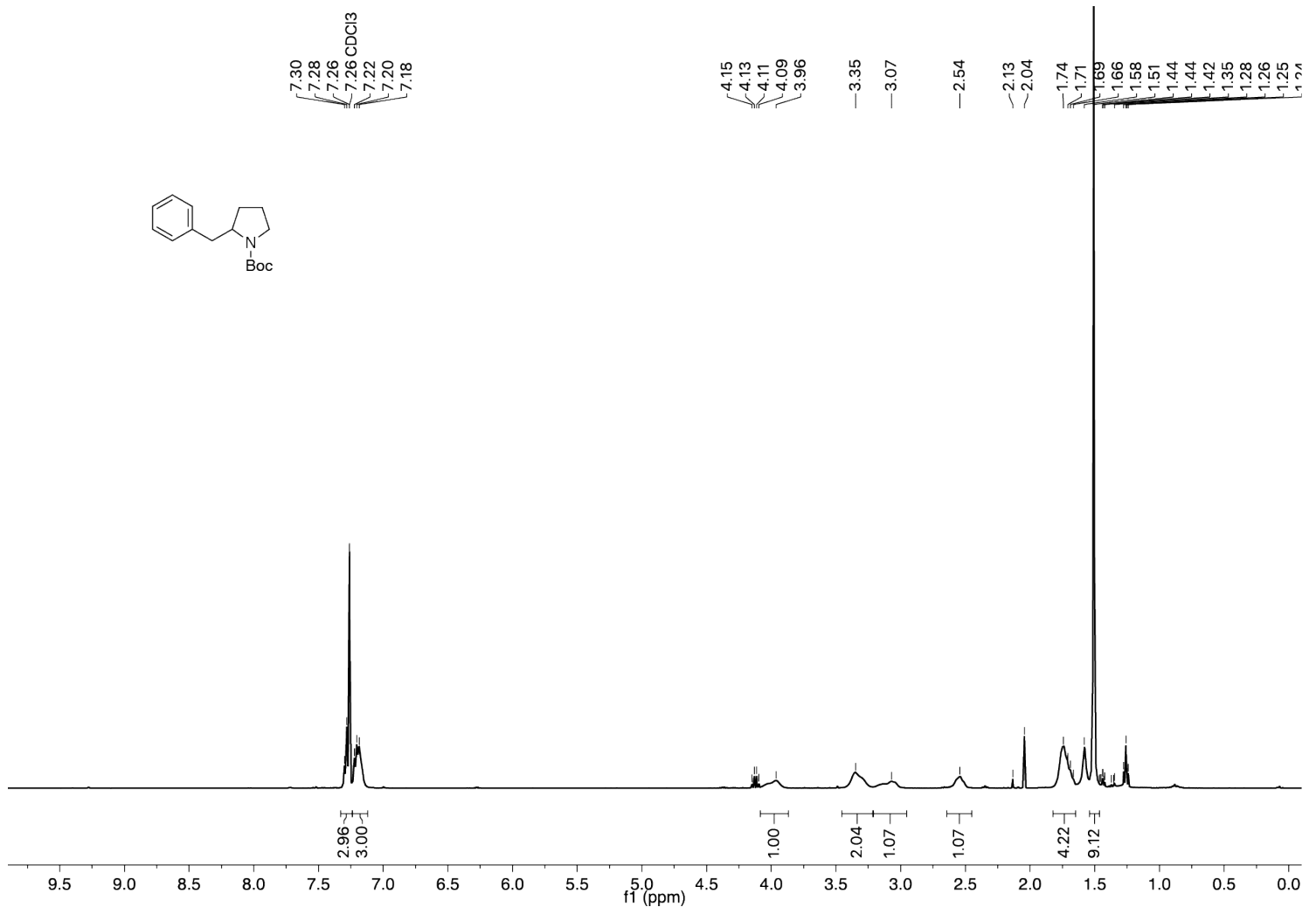
$N$-Boc (3aR, 3a $\left.{ }^{1} R, 5 a S, 10 b S\right)$-8-isopropyl-3a,10b-dimethyl-1,2,3,3a, 3a ${ }^{1}, 4,5,5 a, 6,10 b$-decahydrodibenzo[ $\left.c d, f\right]$ indole (Boc-2m):

${ }^{1} \mathbf{H}$ NMR $\left(400 \mathrm{MHz}, \mathrm{CDCl}_{3}\right.$, rotamer ratio 35:65)
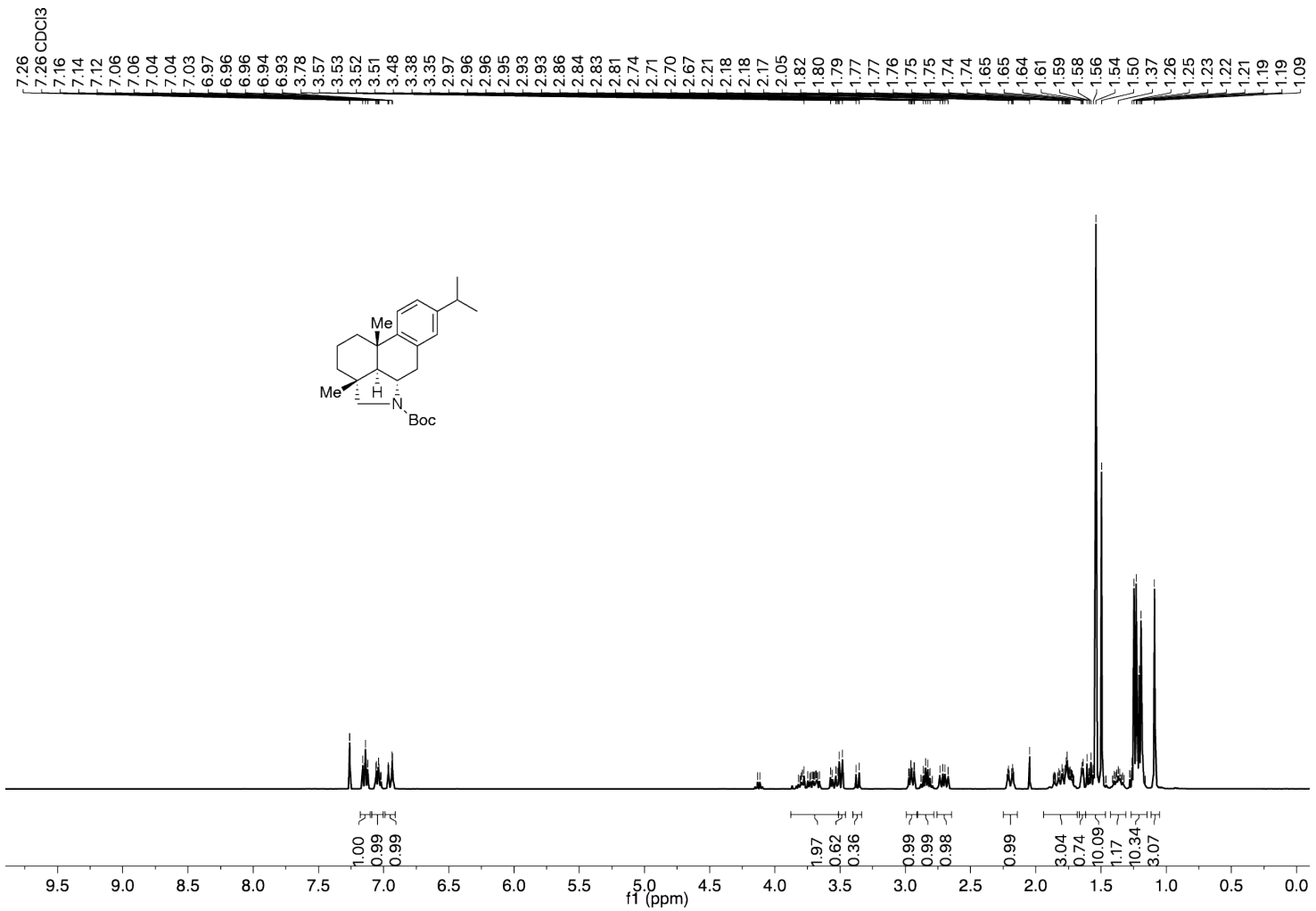

$\operatorname{COSY}\left(400 \mathrm{MHz}, \mathrm{CDCl}_{3}\right.$, rotamer ratio 35:65)

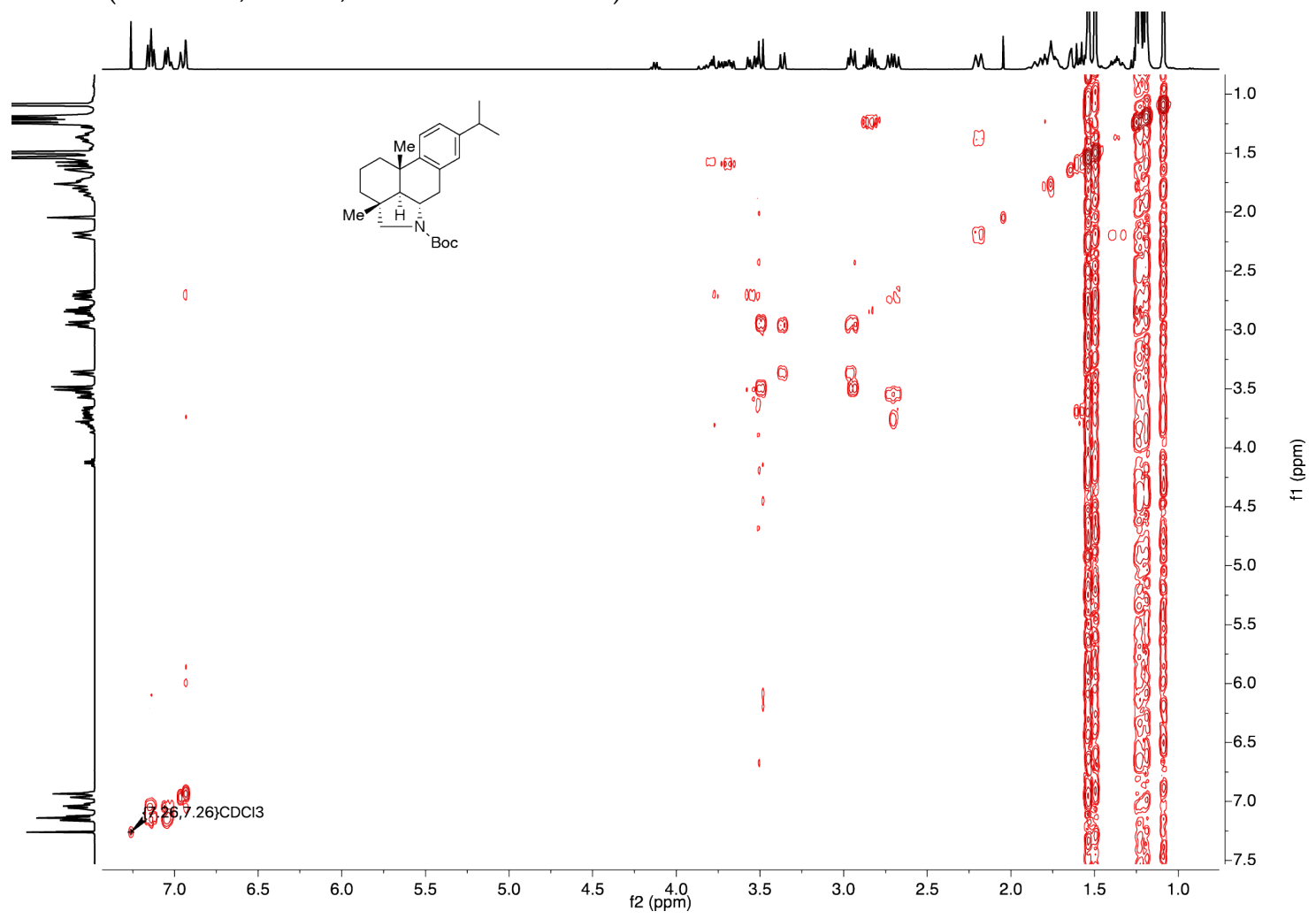

Page S50 of S60 
HSQC (400 MHz, $\mathrm{CDCl}_{3}$, rotamer ratio 35:65)

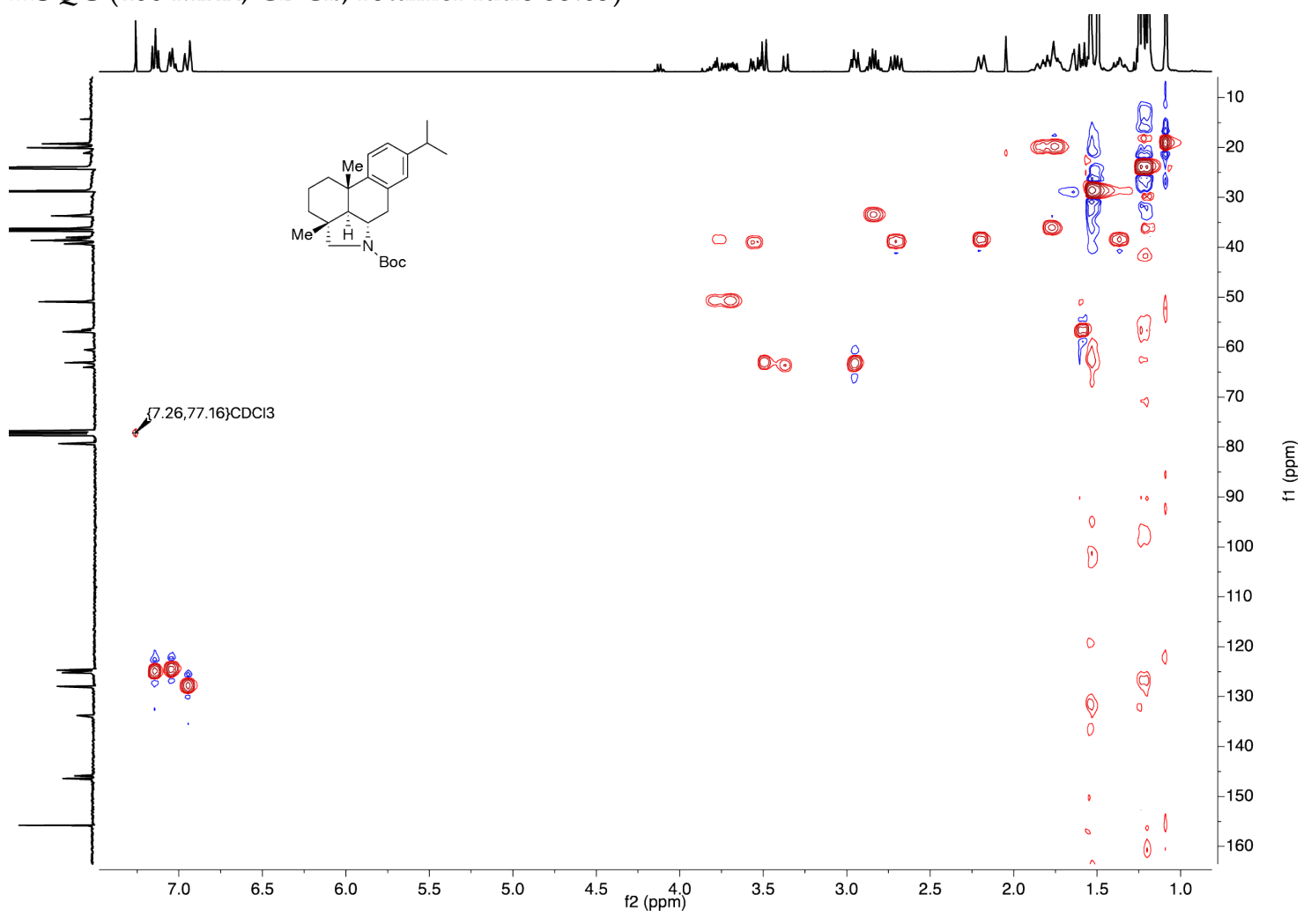


tert-Butyl (benzoyloxy)(3-( $p$-tolyl)propyl)carbamate (Boc-3):

${ }^{1} \mathbf{H}$ NMR $\left(400 \mathrm{MHz}, \mathrm{CDCl}_{3}\right)$
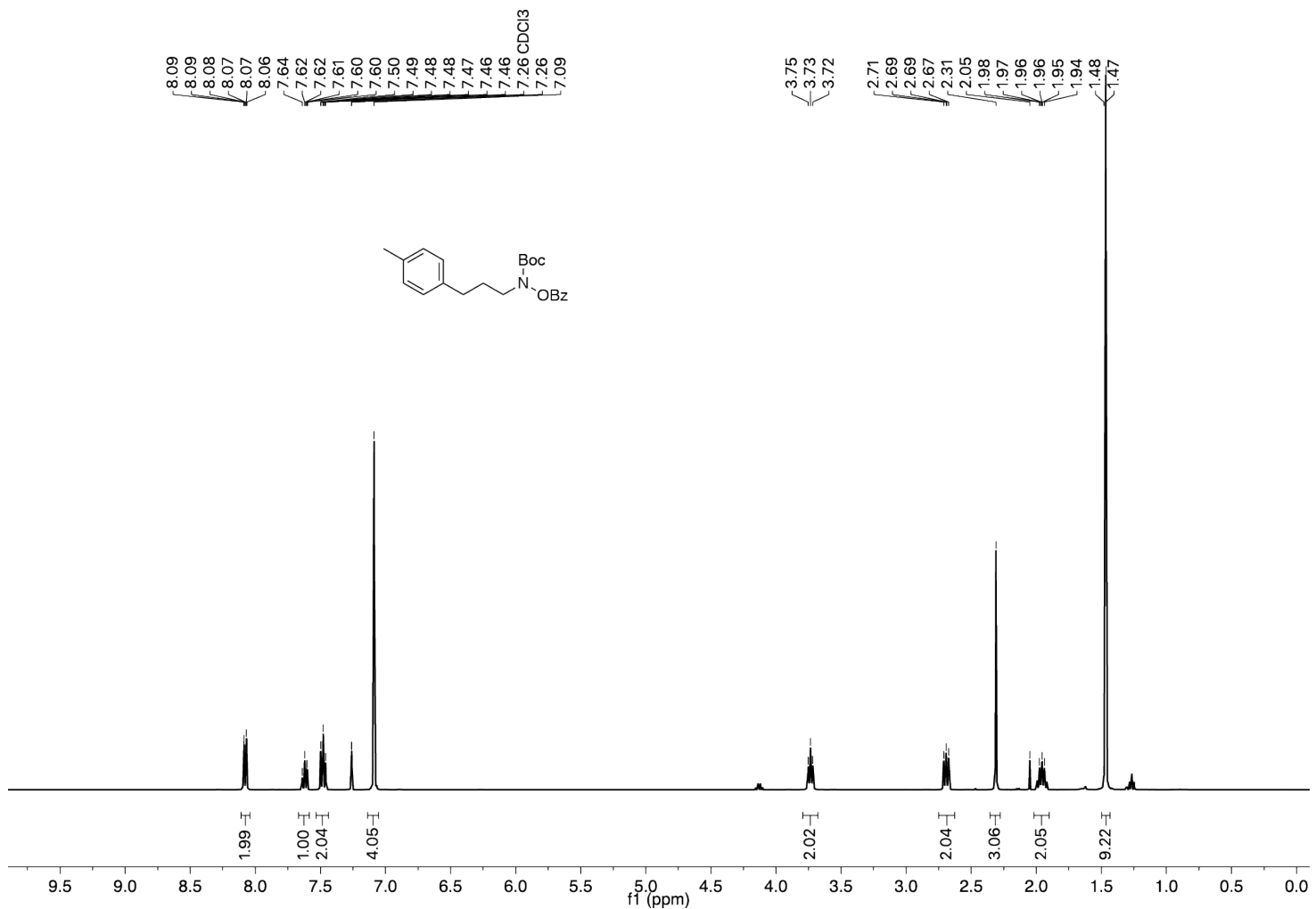

${ }^{13} \mathrm{C}$ NMR $\left(100 \mathrm{MHz}, \mathrm{CDCl}_{3}\right)$
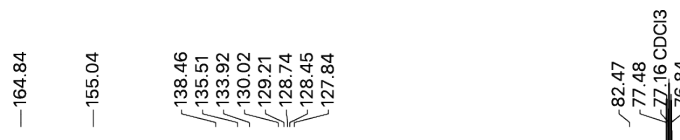

1.
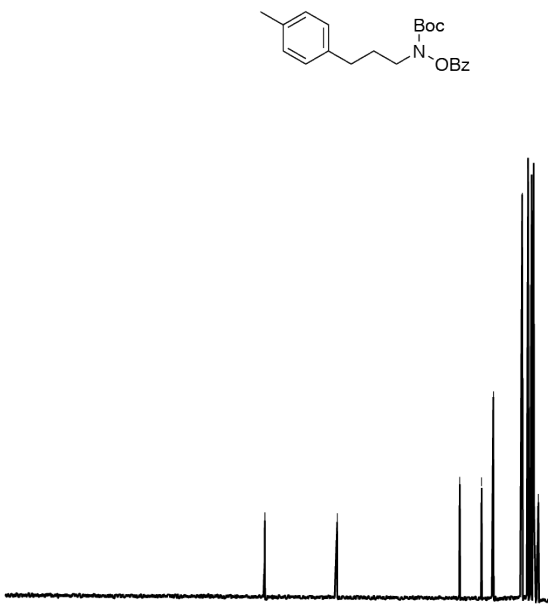

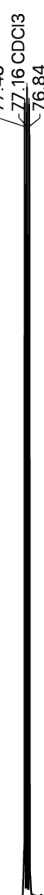

ָָ

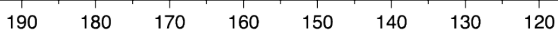

1100

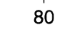

Page S52 of S60 
$O$-Benzoyl- $N$-(3-( $p$-tolyl)propyl)hydroxylamine (3):

${ }^{1} \mathbf{H}$ NMR $\left(400 \mathrm{MHz}, \mathrm{CDCl}_{3}\right)$

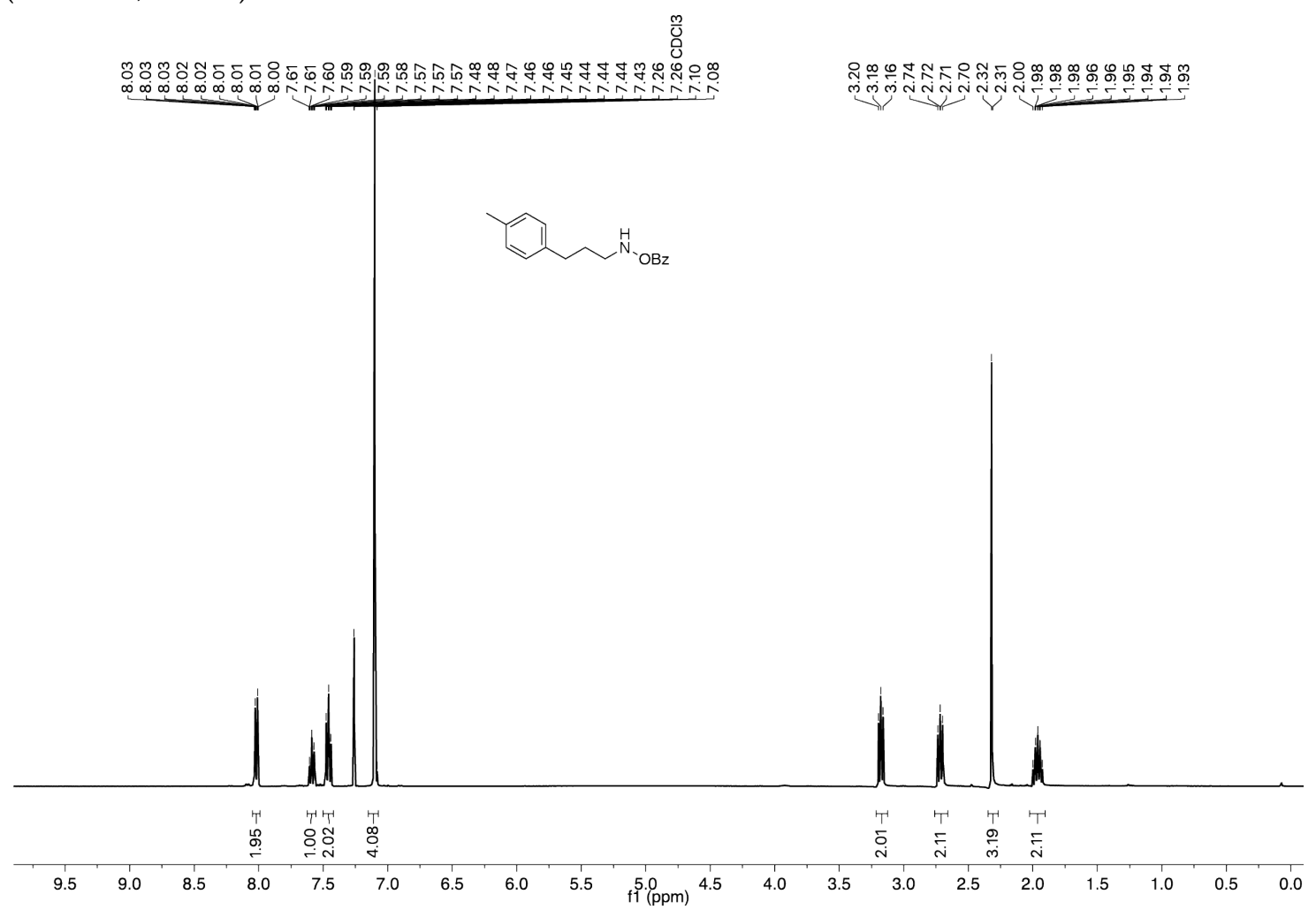

${ }^{13} \mathrm{C}$ NMR (100 MHz, $\left.\mathrm{CDCl}_{3}\right)$

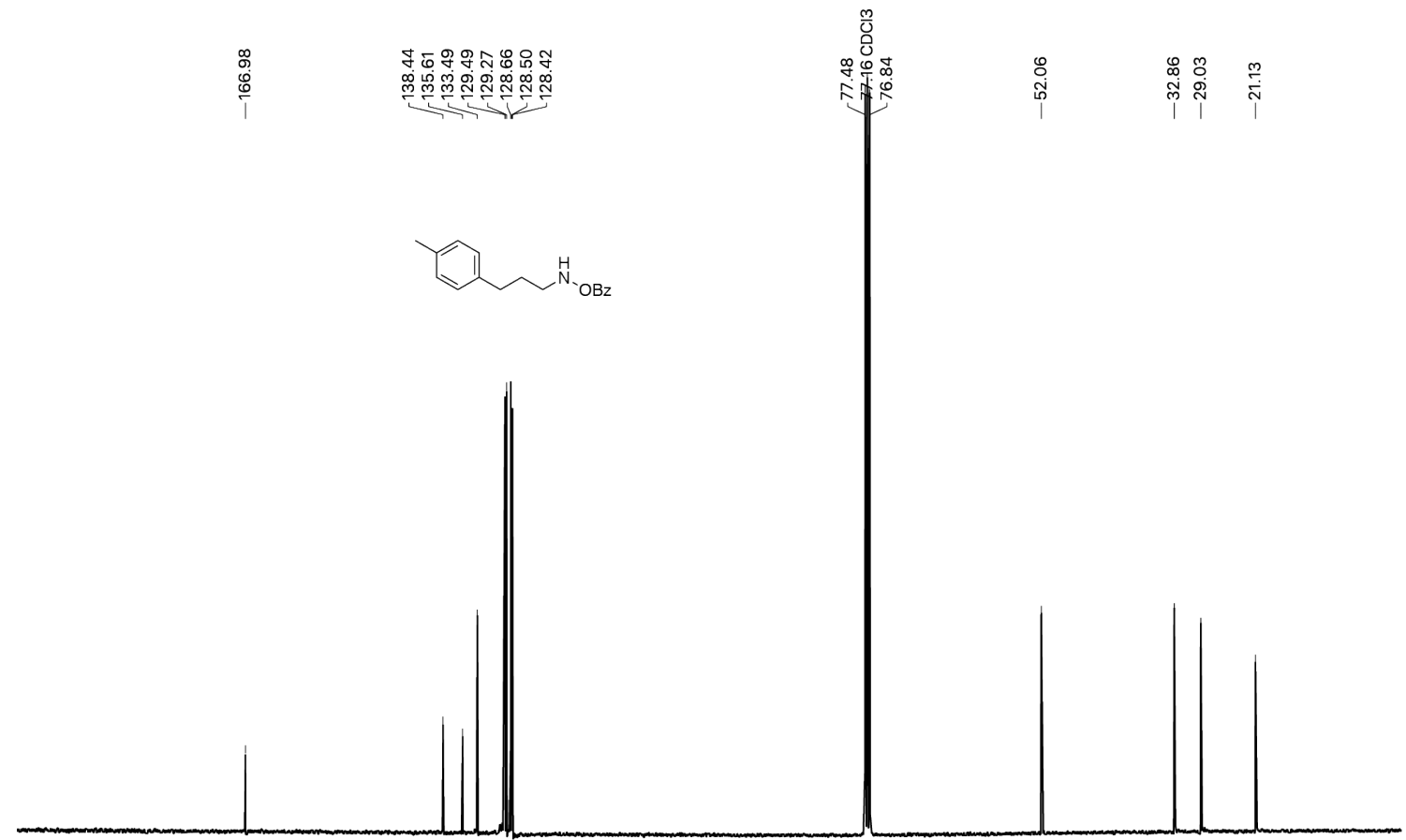

$\begin{array}{llllllll}190 & 180 & 170 & 160 & 150 & 140 & 130 & 120\end{array}$

$110+11000$

$80 \quad 70$

Page S53 of S60 
7-Methyl-1,2,3,4-tetrahydroquinoline (4):

${ }^{1} \mathbf{H}$ NMR $\left(400 \mathrm{MHz}, \mathrm{CDCl}_{3}\right)$

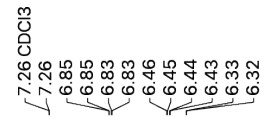

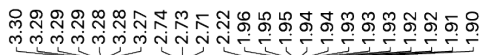

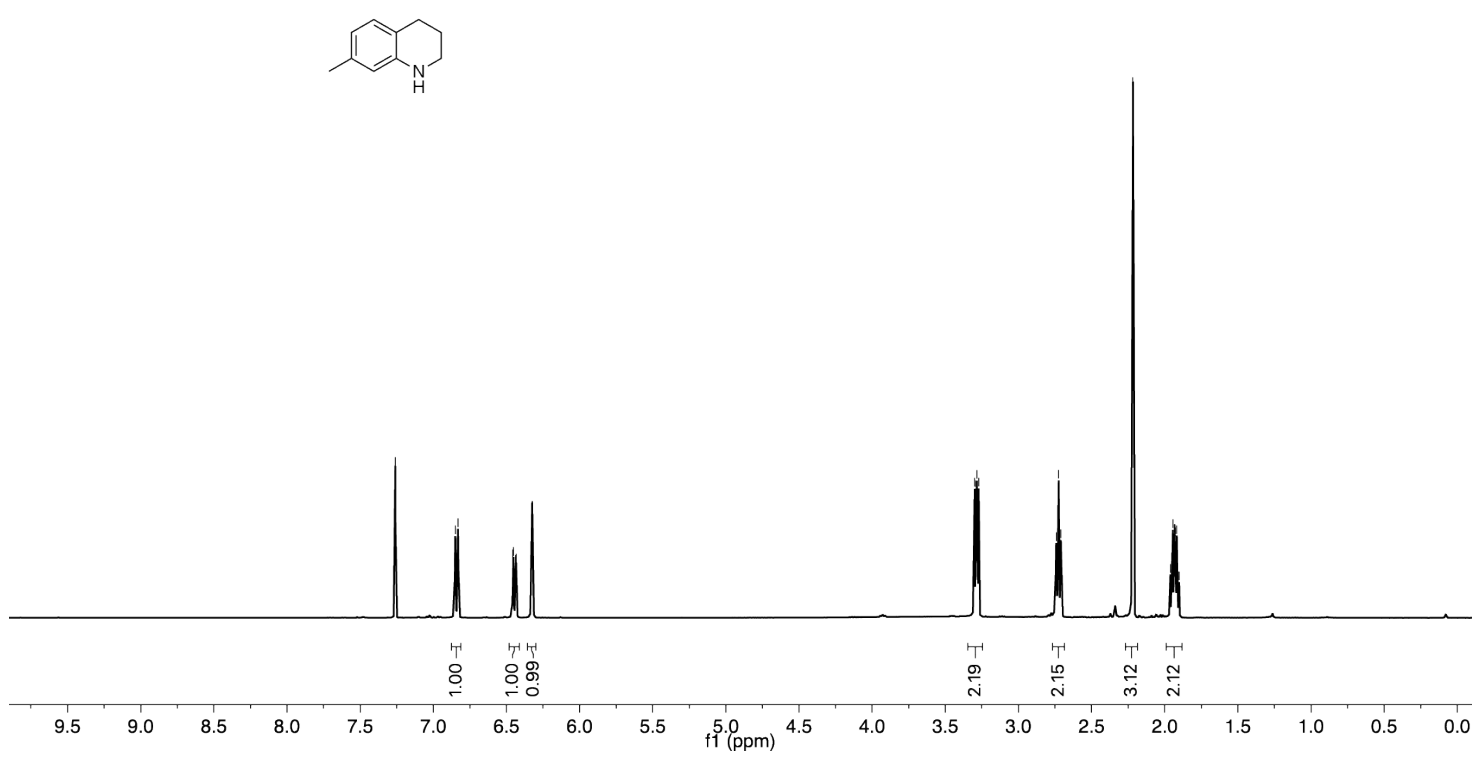

낸 
tert-Butyl (benzoyloxy)(4-(4-fluorophenyl)butyl-4- $d$ )carbamate (Boc-5):

${ }^{1} \mathbf{H}$ NMR (400 MHz, $\left.\mathrm{CDCl}_{3}\right)$
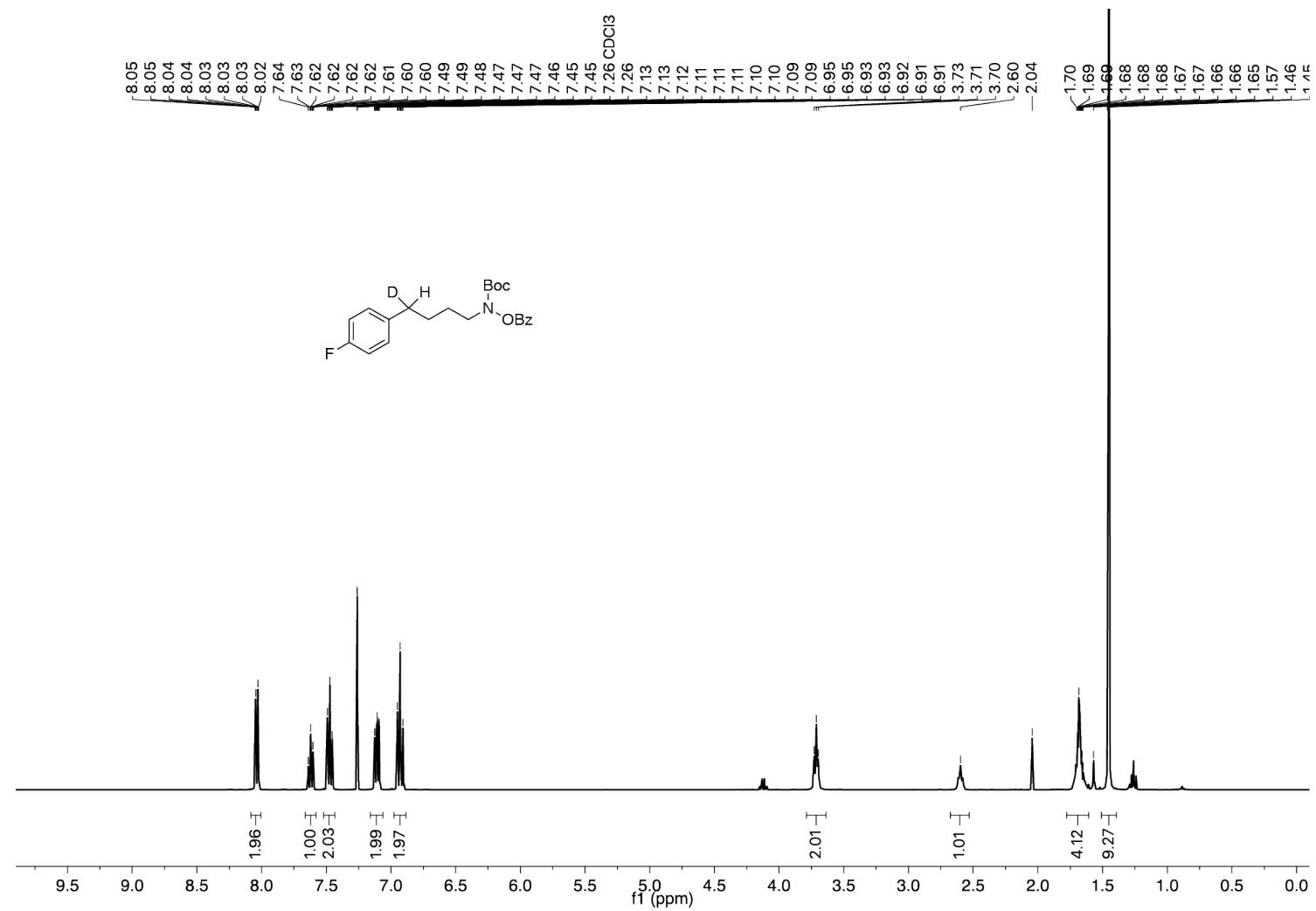

${ }^{13} \mathrm{C}$ NMR $\left(100 \mathrm{MHz}, \mathrm{CDCl}_{3}\right)$

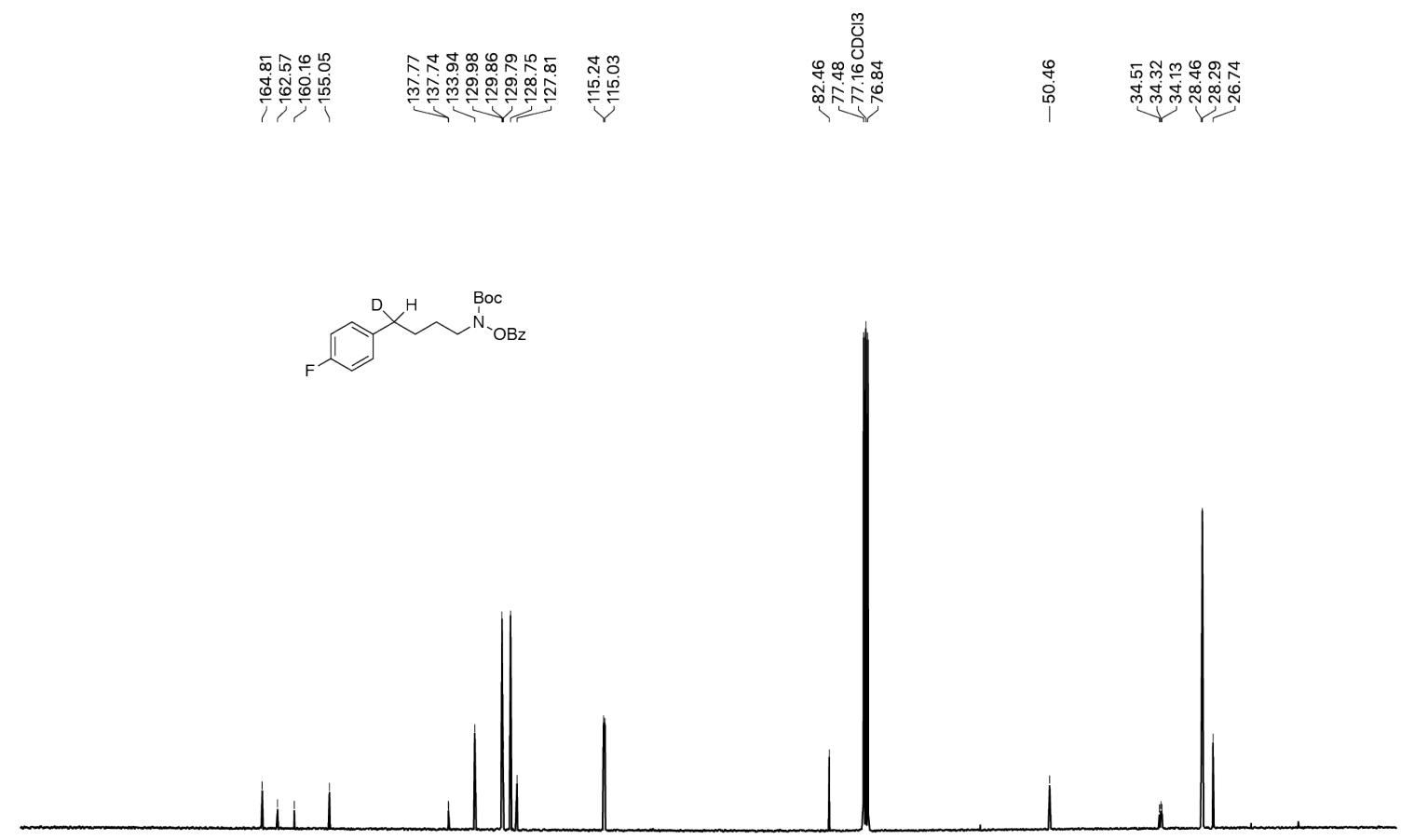

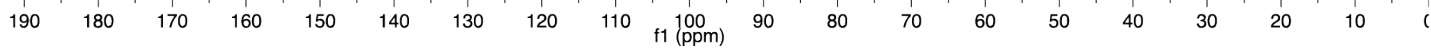

Page S55 of S60 
${ }^{19}$ F NMR (376 MHz, $\left.\mathrm{CDCl}_{3}\right)$

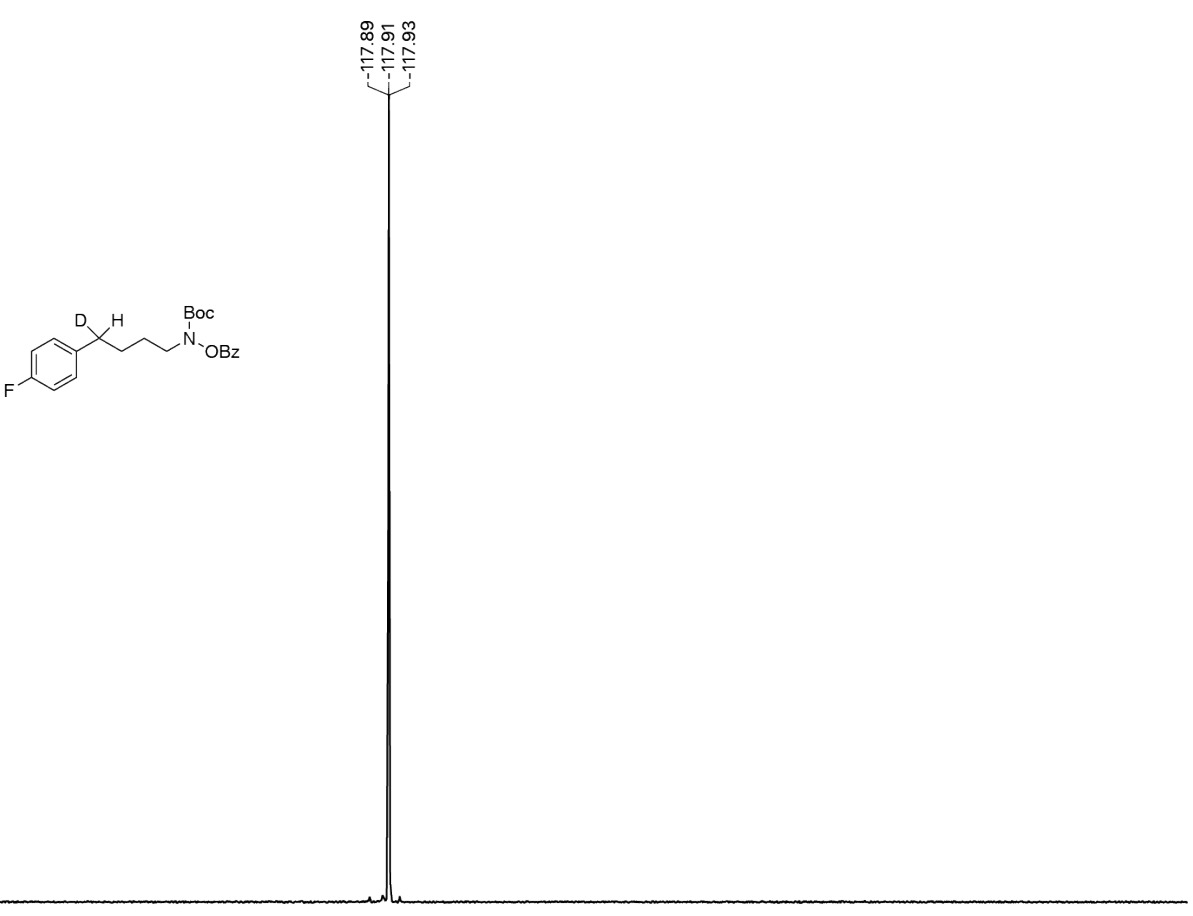

$\begin{array}{llllllllllllllllllllllllllllllllllll}-94 & -96 & -98 & -100 & -102 & -104 & -106 & -108 & -110 & -112 & -114 & -116 & -118 & -120 & -122 & -124 & -126 & -128 & -130 & -132 & -134 & -136 & -138 & -140 & -142 & -144 & -146 & -14\end{array}$ 
$O$-Benzoyl- $N$-(4-(4-fluorophenyl)butyl-4- $d$ )hydroxylamine (5):

${ }^{1} \mathbf{H}$ NMR (400 MHz, $\left.\mathrm{CDCl}_{3}\right)$
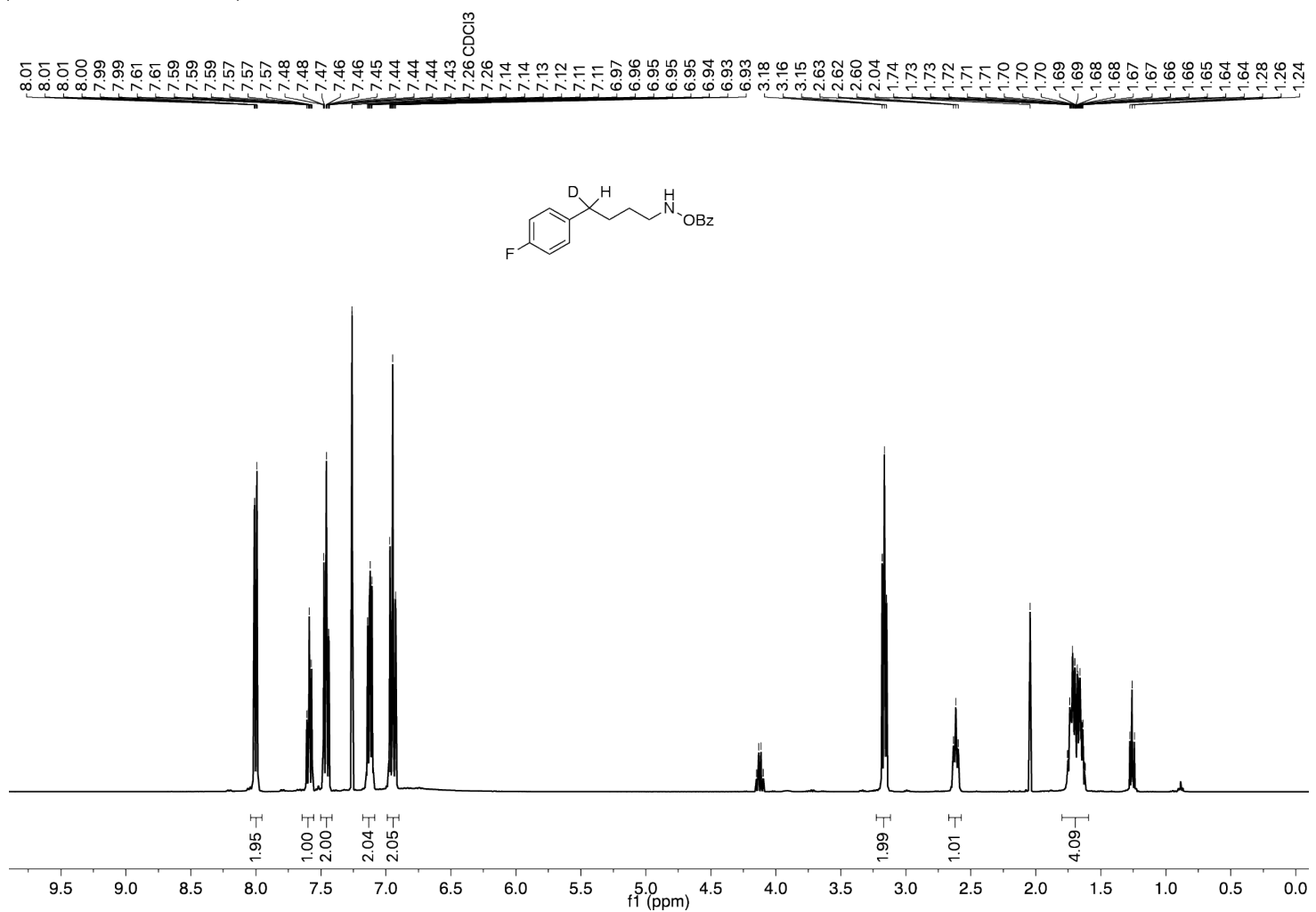

${ }^{13} \mathrm{C}$ NMR (100 MHz, $\left.\mathrm{CDCl}_{3}\right)$

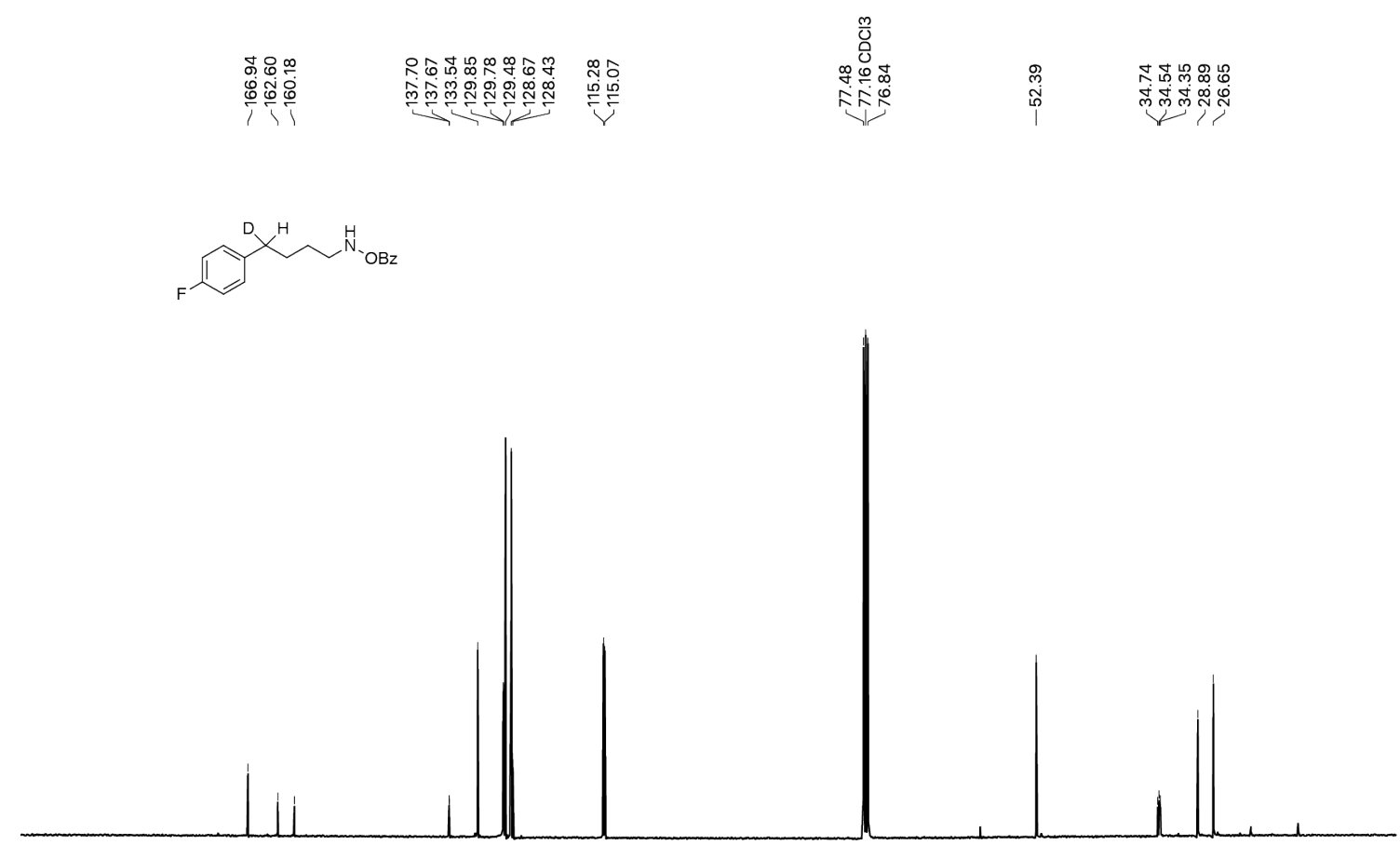

$\begin{array}{llllllll}190 & 180 & 170 & 160 & 150 & 140 & 130 & 120\end{array}$

$110 \underset{f 1}{100}(\mathrm{ppm})$

8070

Page S57 of S60 
${ }^{19}$ F NMR (376 MHz, $\left.\mathrm{CDCl}_{3}\right)$

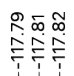

ji
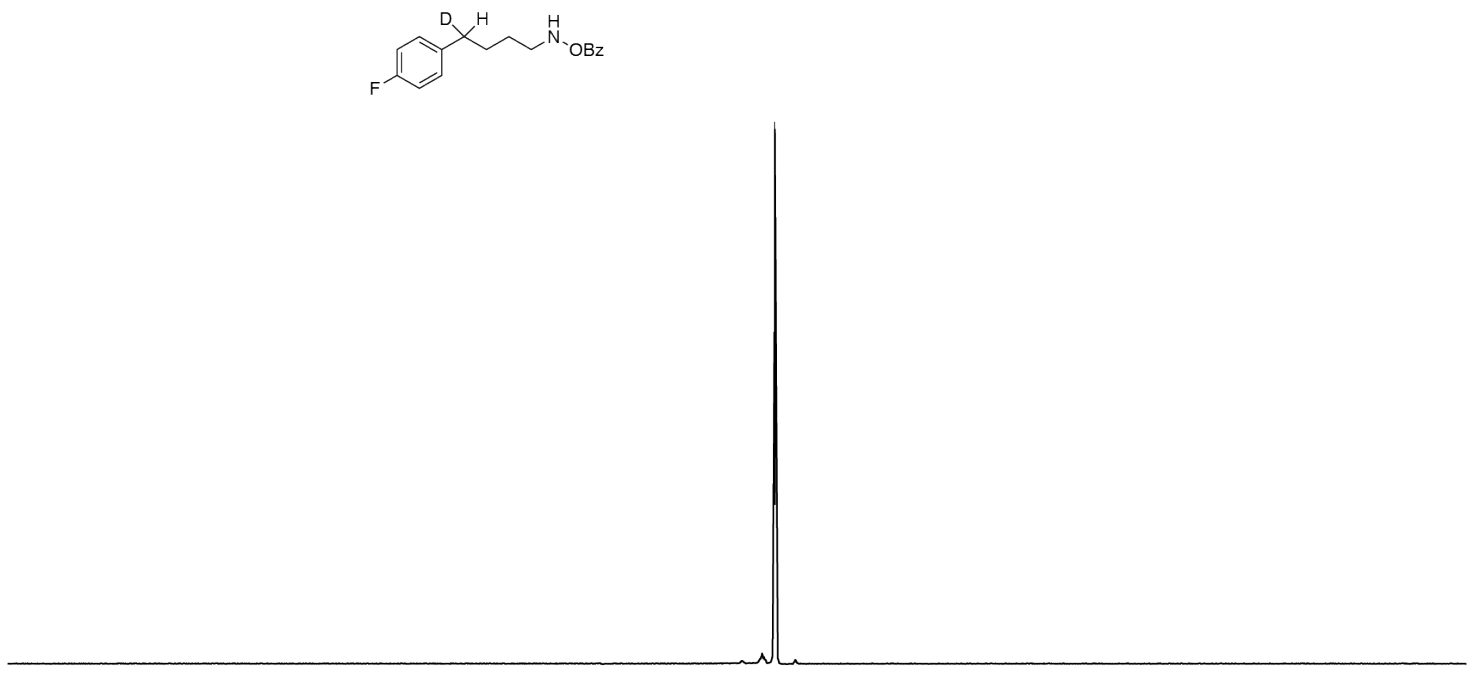

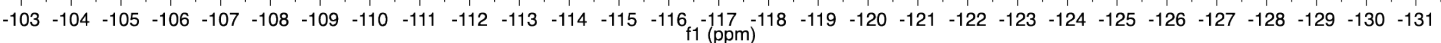


tert-Butyl (benzoyloxy)(4-cyclopropylbutyl)carbamate (Boc-7):

${ }^{1} \mathbf{H}$ NMR (400 MHz, $\mathrm{CDCl}_{3}$ )
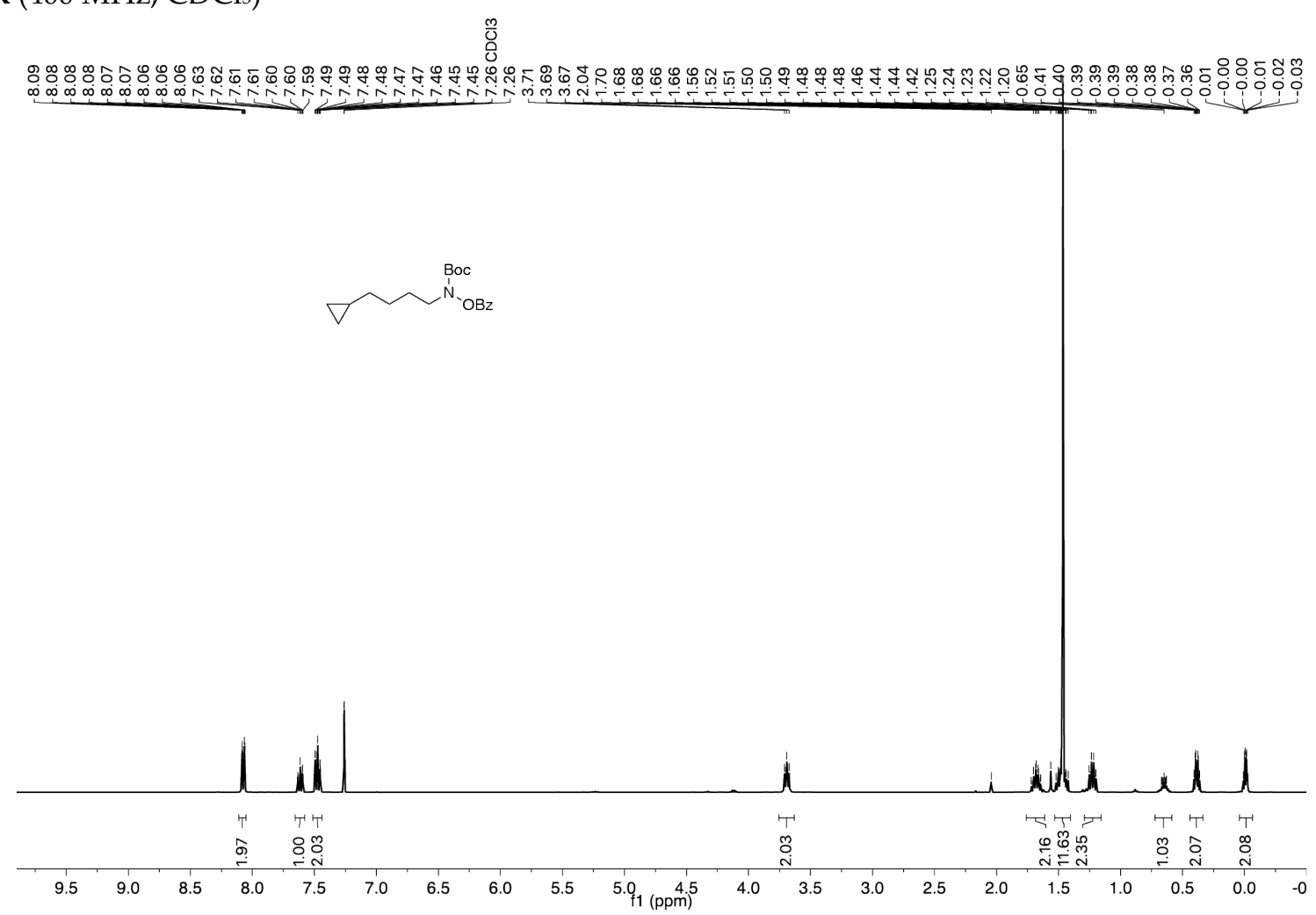

${ }^{13} \mathrm{C}$ NMR (100 MHz, $\left.\mathrm{CDCl}_{3}\right)$

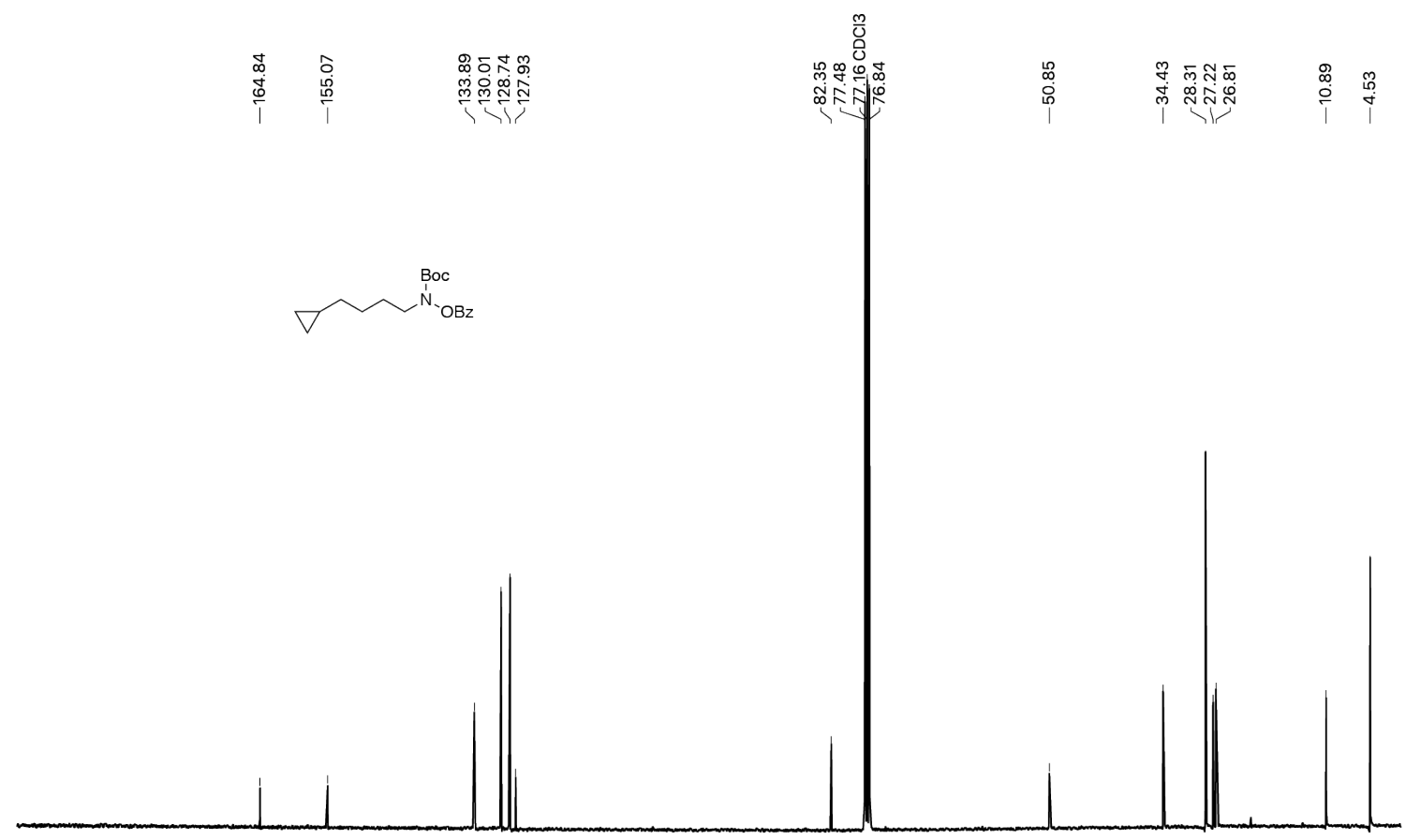

$\begin{array}{llllllll}190 & 180 & 170 & 160 & 150 & 140 & 130 & 120\end{array}$

$110+11000$

Page S59 of S60 
O-Benzoyl- $N$-(4-cyclopropylbutyl)hydroxylamine (7):

${ }^{1} \mathbf{H}$ NMR $\left(400 \mathrm{MHz}, \mathrm{CDCl}_{3}\right)$
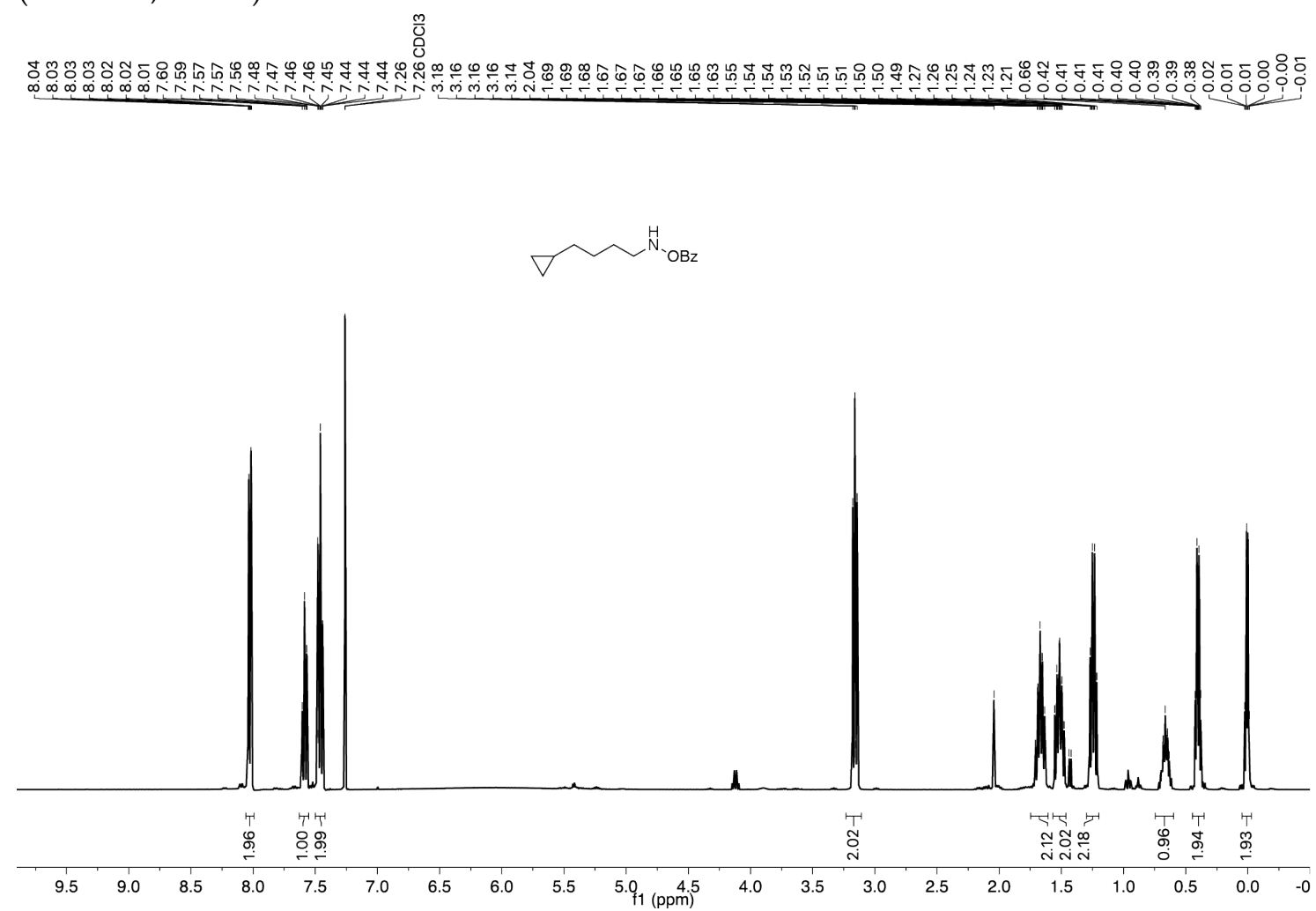

${ }^{13} \mathrm{C}$ NMR (100 MHz, $\left.\mathrm{CDCl}_{3}\right)$
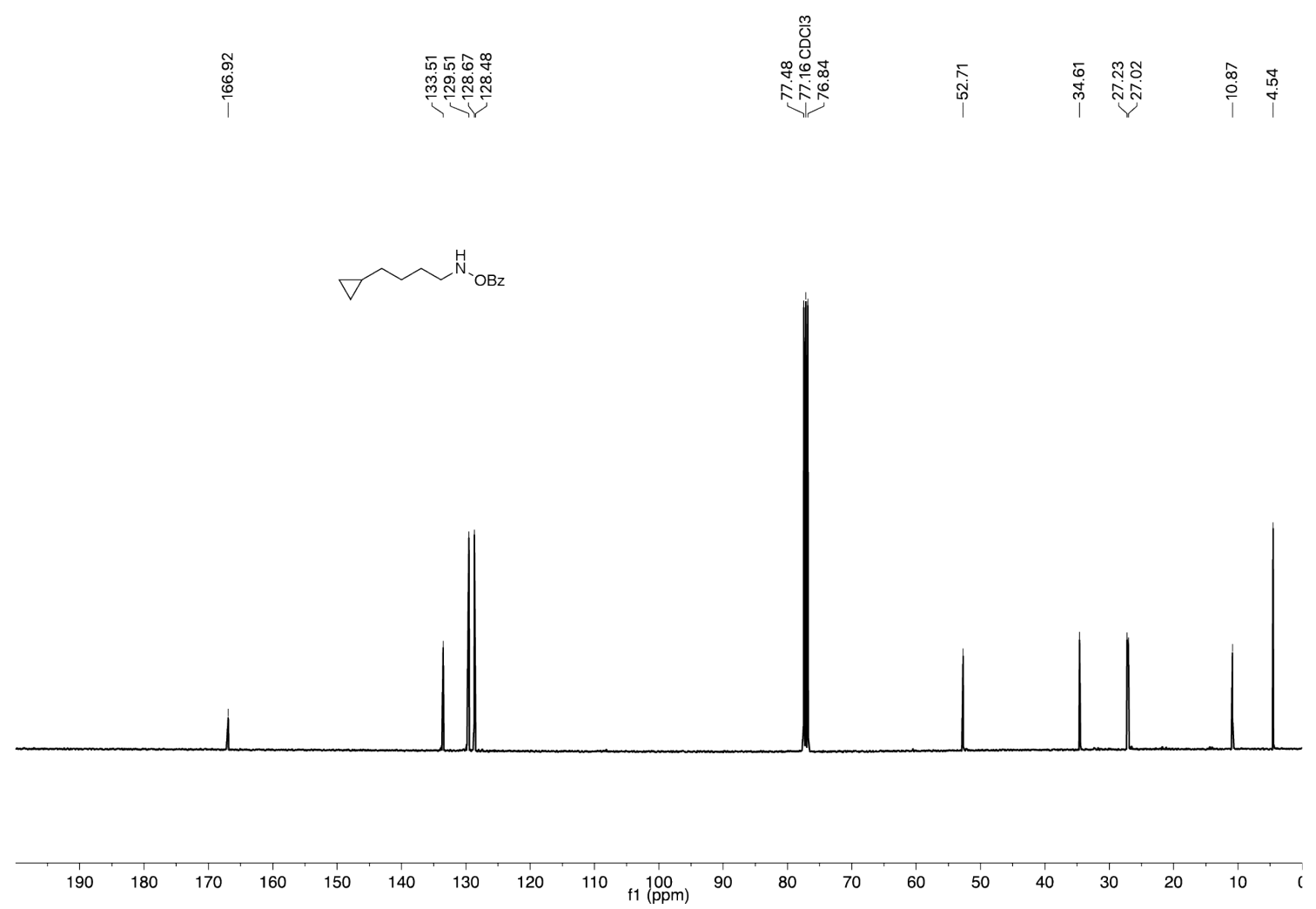

Page S60 of S60 